\title{
Inhibition of C(2)-H Activity on Alkylated Imidazolium Monocations and Dications Upon Inclusion in Cucurbit[7]uril
}

Shengke Li, ${ }^{\dagger}$ Ian W. Wyman, ${ }^{\ddagger}$ Chunming Wang, ${ }^{\dagger}$ Yitao Wang, ${ }^{\dagger}$ Donal H. Macartney, ${ }^{\ddagger * *}$ and Ruibing Wang ${ }^{\dagger, *}$

\section{Supporting Information}

Figure S1. The ${ }^{1} \mathrm{H}$ NMR spectra of the free DIC4 ${ }^{2+}$ guest (lower spectrum) and the spectra of $\mathrm{DIC}^{2+}{ }^{2}$ recorded in the presence of 0.3 equiv. $\mathrm{CB}[7]$ (middle) and 1.2 equiv. $\mathrm{CB}[7]$ (top).

Figure S2. The ${ }^{1} \mathrm{H}$ NMR spectra of the free $\mathrm{IC}^{+}$guest (lower spectrum) and the spectra of $\mathrm{IC}^{+}$recorded in the presence of 0.3 equiv. $\mathrm{CB}$ [7] (middle) and 1.2 equiv. $\mathrm{CB}[7]$ (top).

Figure S3. The ${ }^{1} \mathrm{H}$ NMR spectra of the free $\mathrm{DIC} 8^{2+}$ guest (lower spectrum) and the spectra of DIC $8^{2+}$ recorded in the presence of 0.3 equiv. $\mathrm{CB}$ [7] (middle) and 1.2 equiv. $\mathrm{CB}[7]$ (top).

Figure S4. The ${ }^{1} \mathrm{H}$ NMR spectra of the free $\mathrm{IC} 8^{+}$guest (lower spectrum) and of $\mathrm{IC}^{+}$recorded in the presence of 0.3 equiv. $\mathrm{CB}$ [7] (middle) and 1.2 equiv. $\mathrm{CB}[7]$ (top).

Figure S5. Electrospray ionization mass spectrum of $\{\mathrm{DIC} 4 \cdot \mathrm{CB}[7]\}^{2+}$.

Figure S6. Electrospray ionization mass spectrum of $\{\mathrm{DIC} 6 \cdot \mathrm{CB}[7]\}^{2+}$. 8

Figure S7. Electrospray ionization mass spectrum of $\{\mathrm{DIC} 8 \cdot \mathrm{CB}[7]\}^{2+}$. 9

Figure S8. Electrospray ionization mass spectrum of $\{\mathrm{IC} 4 \cdot \mathrm{CB}[7]\}^{+}$. 10

Figure S9. Electrospray ionization mass spectrum of $\left\{\mathrm{IC} 6 \bullet^{\circ} \mathrm{CB}[7]\right\}^{+}$. 11

Figure S10. Electrospray ionization mass spectrum of $\{\mathrm{IC} 8 \cdot \mathrm{CB}[7]\}^{+}$. 12

Figure S11. Job plots for DIC4 ${ }^{2+}$ and $\mathrm{IC}^{+}$. 13

Figure S12. Job plots for DIC $8^{2+}$ and $\mathrm{IC}^{+}$. 14

Figure S13. The ${ }^{1} \mathrm{H}$ NMR spectrum of competitive titration for $\mathrm{DIC} 4^{2+}$ upon complexation with $\mathrm{CB}[7]$ in the presence of TMSP.

Figure S14. The ${ }^{1} \mathrm{H}$ NMR spectrum of competitive titration for $\mathrm{DIC}^{2+}$ upon complexation with $\mathrm{CB}[7]$ in the presence of TMSP.

Figure S15. The ${ }^{1} \mathrm{H}$ NMR spectrum of competitive titration for $\mathrm{DIC} 8^{2+}$ upon complexation with $\mathrm{CB}[7]$ in the presence of TMSP.

Figure S16. The ${ }^{1} \mathrm{H}$ NMR spectrum of competitive titration for $\mathrm{IC}^{+}$upon complexation with $\mathrm{CB}[7]$ in the presence of TMSP.

Figure S17. The ${ }^{1} \mathrm{H}$ NMR spectrum of competitive titration for $\mathrm{IC}^{+}$upon complexation with $\mathrm{CB}[7]$ in the presence of TMSP.

Figure S18. The ${ }^{1} \mathrm{H}$ NMR spectrum of competitive titration for $\mathrm{IC}^{+}$upon complexation with $\mathrm{CB}[7]$ in the presence of TMSP.

Determinations of Guest-Host Binding Constant

Figure S19. Semilogarithmic plot of $\ln R$ against time for the $\mathrm{C}(2)$-proton deuterium exchange of DIC4 $4^{2+}$

Figure S20. Semilogarithmic plot of $\ln R$ against time for the $\mathrm{C}(2)$-proton 
deuterium exchange of DIC6 ${ }^{2+}$

Figure S21. Semilogarithmic plot of $\ln R$ against time for the $\mathrm{C}(2)$-proton deuterium exchange of DIC $8^{2+}$

Figure S22. Semilogarithmic plot of $\ln R$ against time for the $\mathrm{C}(2)$-proton deuterium exchange of $\mathrm{IC}^{+}$

Figure S23. Semilogarithmic plot of $\ln R$ against time for the $\mathrm{C}(2)$-proton deuterium exchange of $\mathrm{IC}^{+}$

Figure S24. Semilogarithmic plot of $\ln R$ against time for the $\mathrm{C}(2)$-proton deuterium exchange of $\mathrm{IC}^{+}$

Determination of the first-order rate constants by semilogarithmic

plots

Determination of the second-order rate constants

Figure S25. Plots of $\log k_{\mathrm{ex}}$ against $\mathrm{p} D$ for the $\mathrm{C}(2)-\mathrm{H} / \mathrm{D}$ exchange reactions for the dications and monocations

Figure S26. Energy-minimized structures (HF/3-21G** basis set) of

$\{\mathrm{DIC} 4 \cdot \mathrm{CB}[7]\}^{2+},\{\mathrm{IC} 4 \cdot \mathrm{CB}[7]\}^{+},\{\mathrm{DIC} 8 \cdot \mathrm{CB}[7]\}^{2+}$ and $\{\mathrm{IC} 8 \cdot \mathrm{CB}[7]\}^{+} \quad 26$

The H-bonding length and angles 26

Full Citation for Reference 24

Absolute energies (in Hartrees) and coordinates of the atoms of the CB [7] complexes 

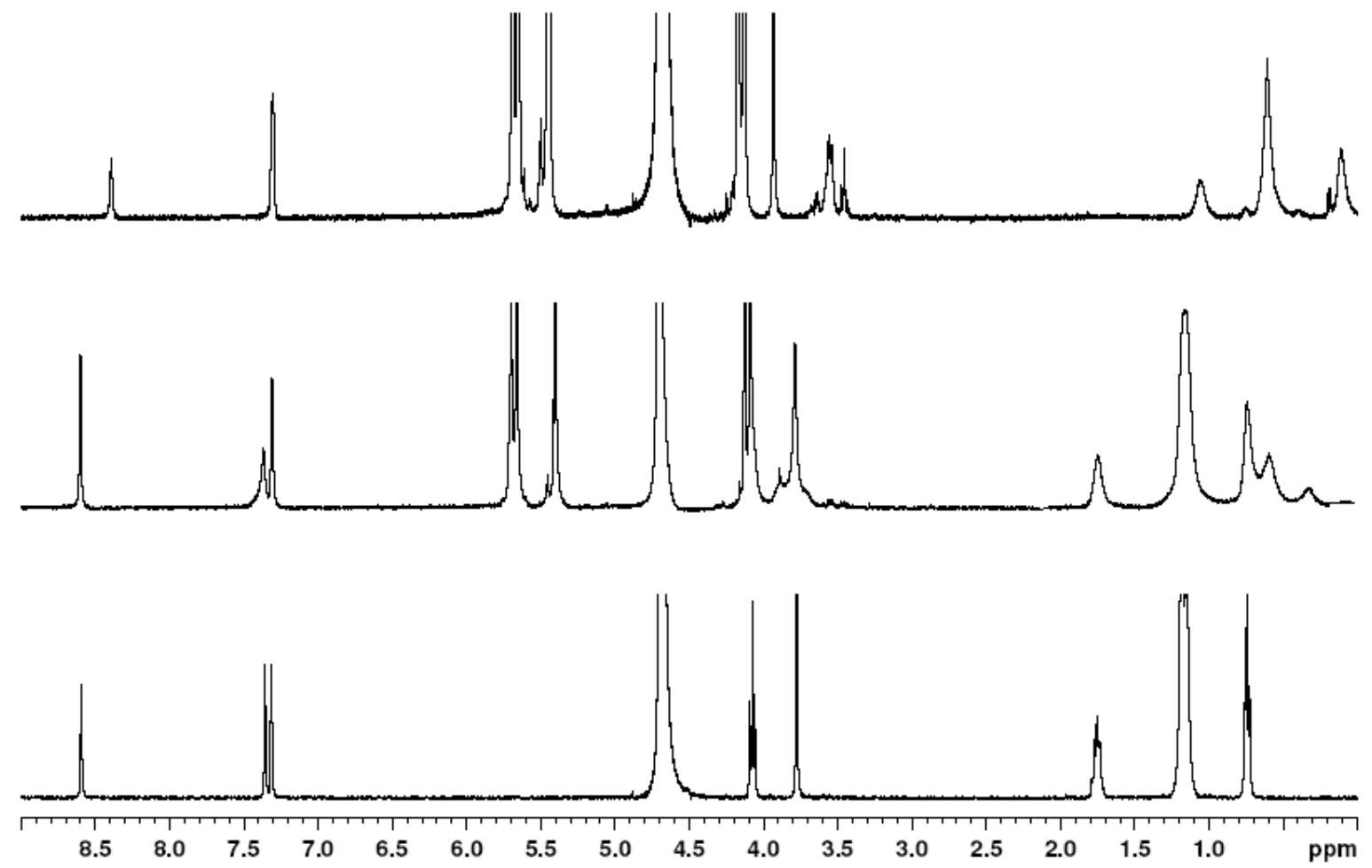

Figure S1. The ${ }^{1} \mathrm{H}$ NMR spectra of the free $\mathrm{DIC} 4^{2+}$ guest (lower spectrum) and the spectra of DIC4 $4^{2+}$ recorded in the presence of 0.3 equiv. $\mathrm{CB}$ [7] (middle) and 1.2 equiv. $\mathrm{CB}[7]$ (top). 

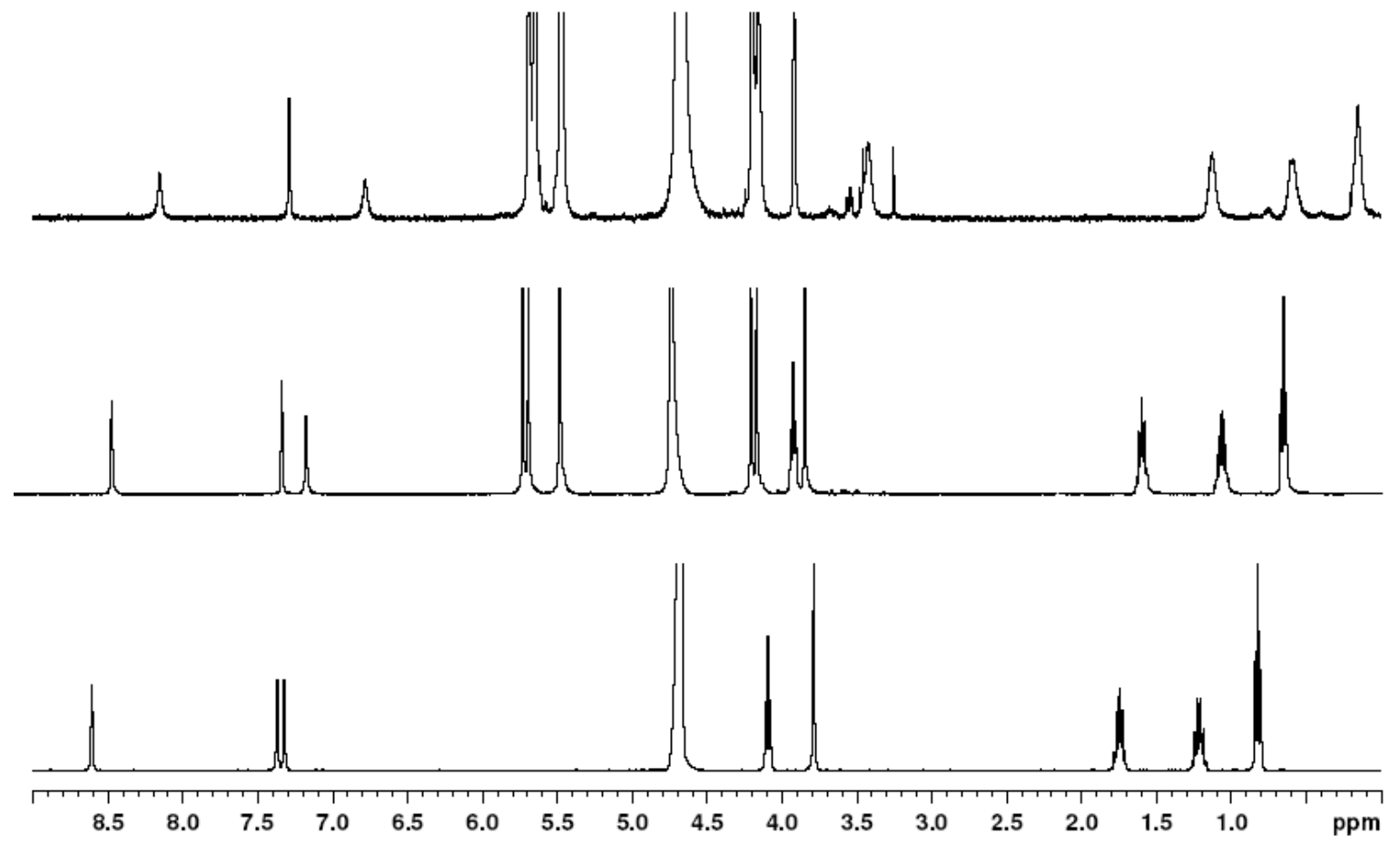
Figure S2. The ${ }^{1} \mathrm{H}$ NMR spectra of the free $\mathrm{IC}^{+}$guest (lower spectrum) and the spectra of $\mathrm{IC}^{+}$recorded in the presence of 0.3 equiv. $\mathrm{CB}$ [7] (middle) and 1.2 equiv. $\mathrm{CB}$ [7] (top).
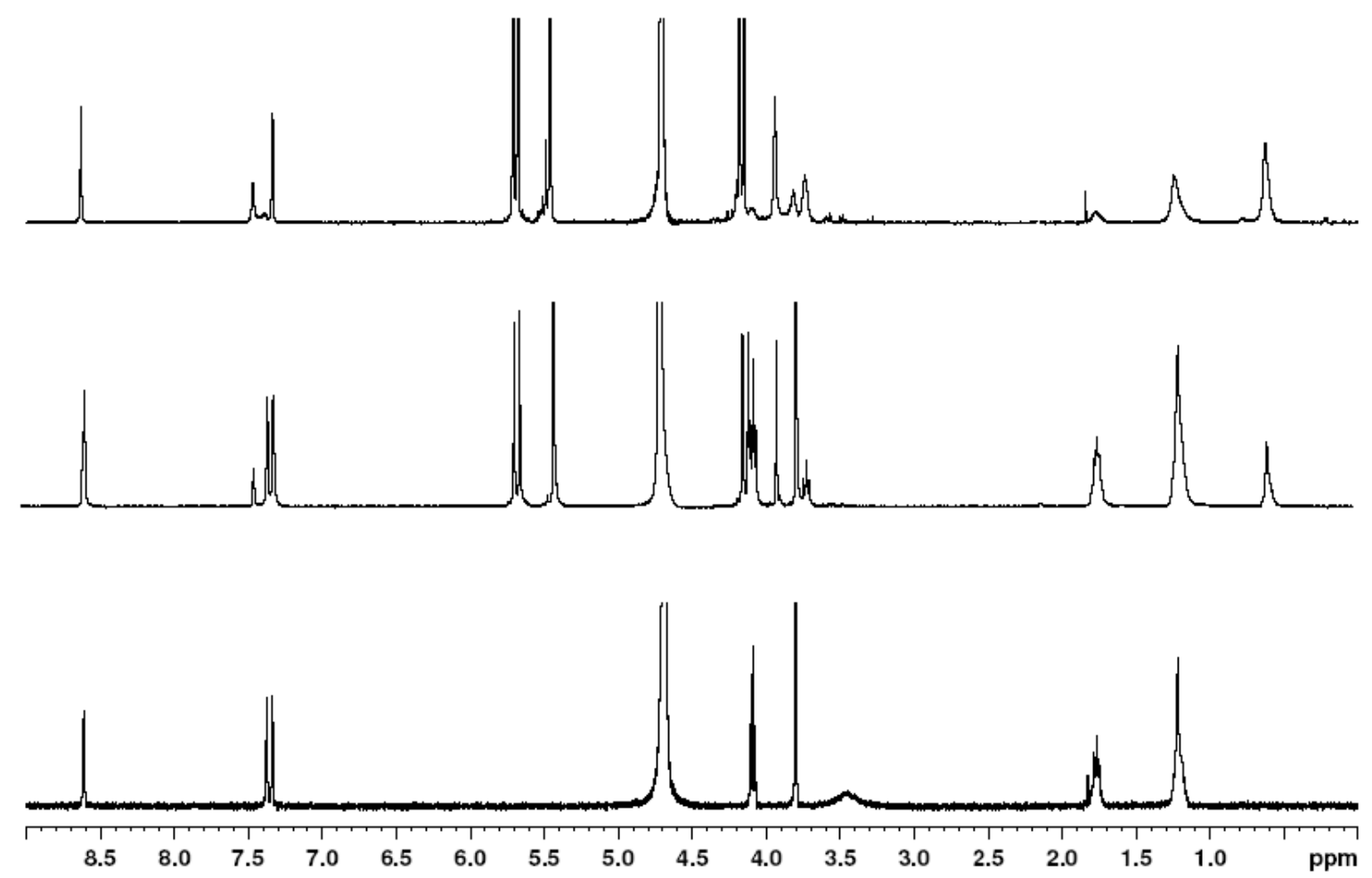

Figure S3. The ${ }^{1} \mathrm{H}$ NMR spectra of the free DIC $8^{2+}$ guest (lower spectrum) and the spectra of DIC $8^{2+}$ recorded in the presence of 0.3 equiv. CB[7] (middle) and 1.2 equiv. $\mathrm{CB}[7]$ (top). 

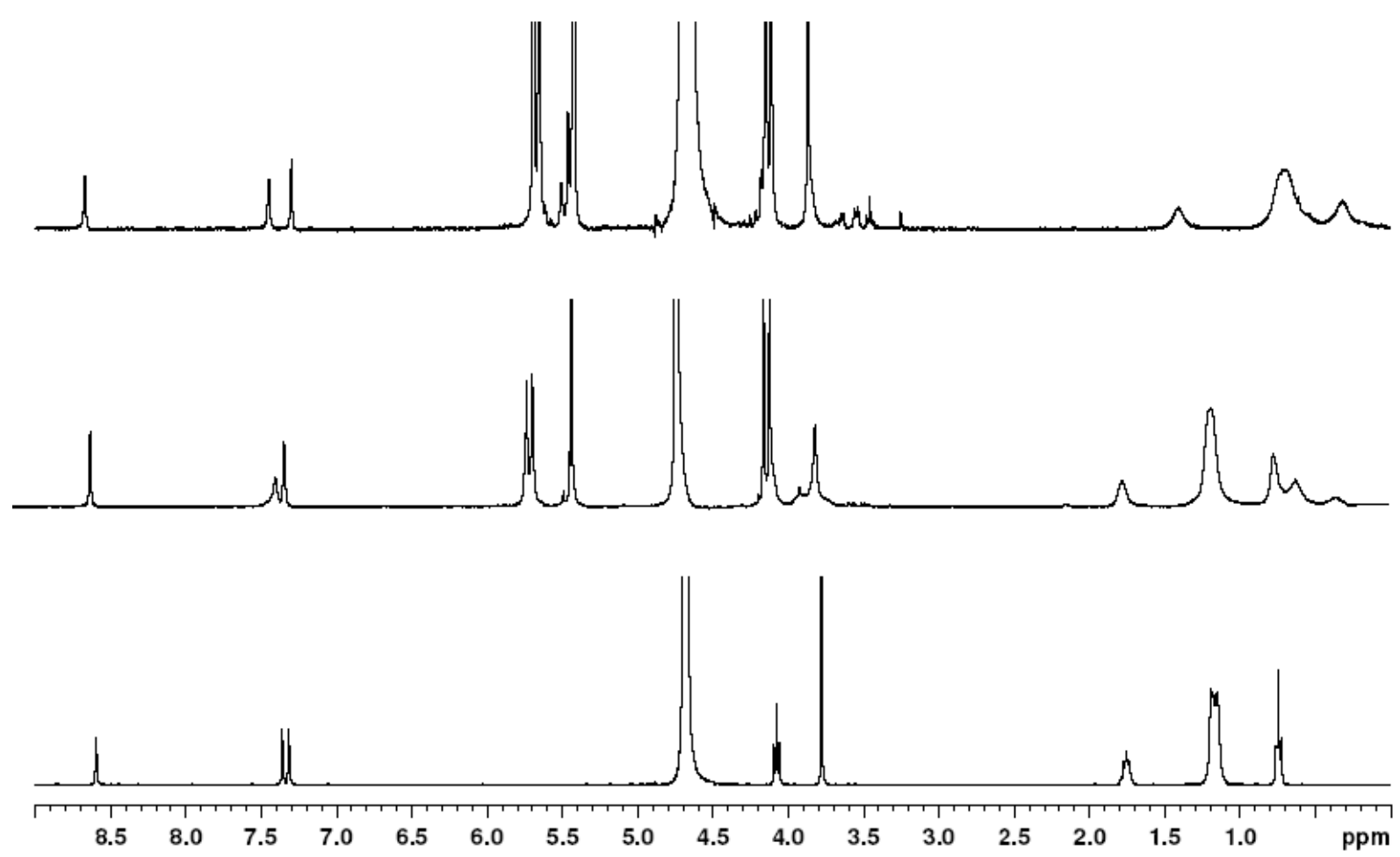

Figure S4. The ${ }^{1} \mathrm{H}$ NMR spectra of the free $\mathrm{IC} 8^{+}$guest (lower spectrum) and of IC $8^{+}$ recorded in the presence of 0.3 equiv. $\mathrm{CB}$ [7] (middle) and 1.2 equiv. $\mathrm{CB}$ [7] (top). 


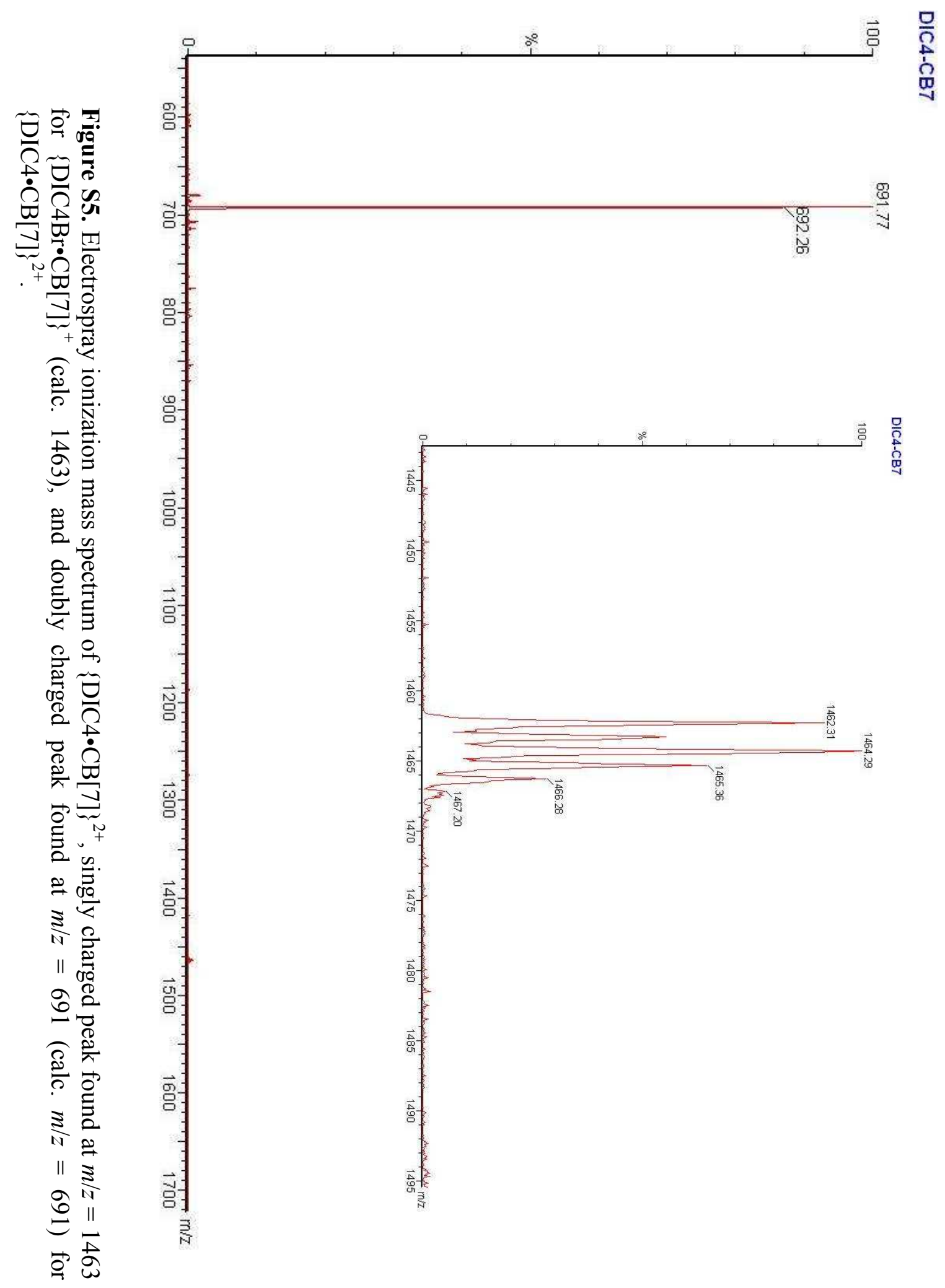




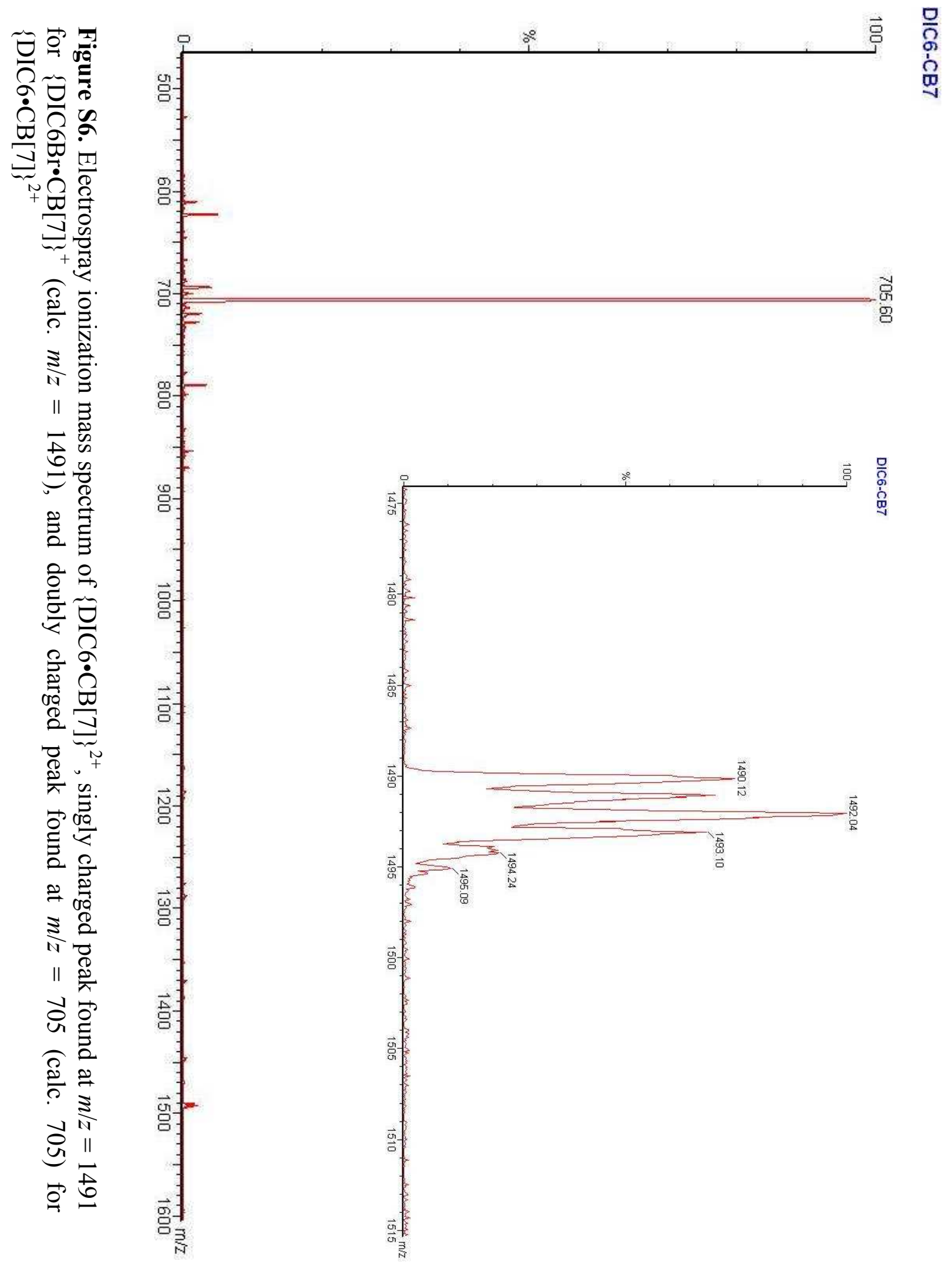




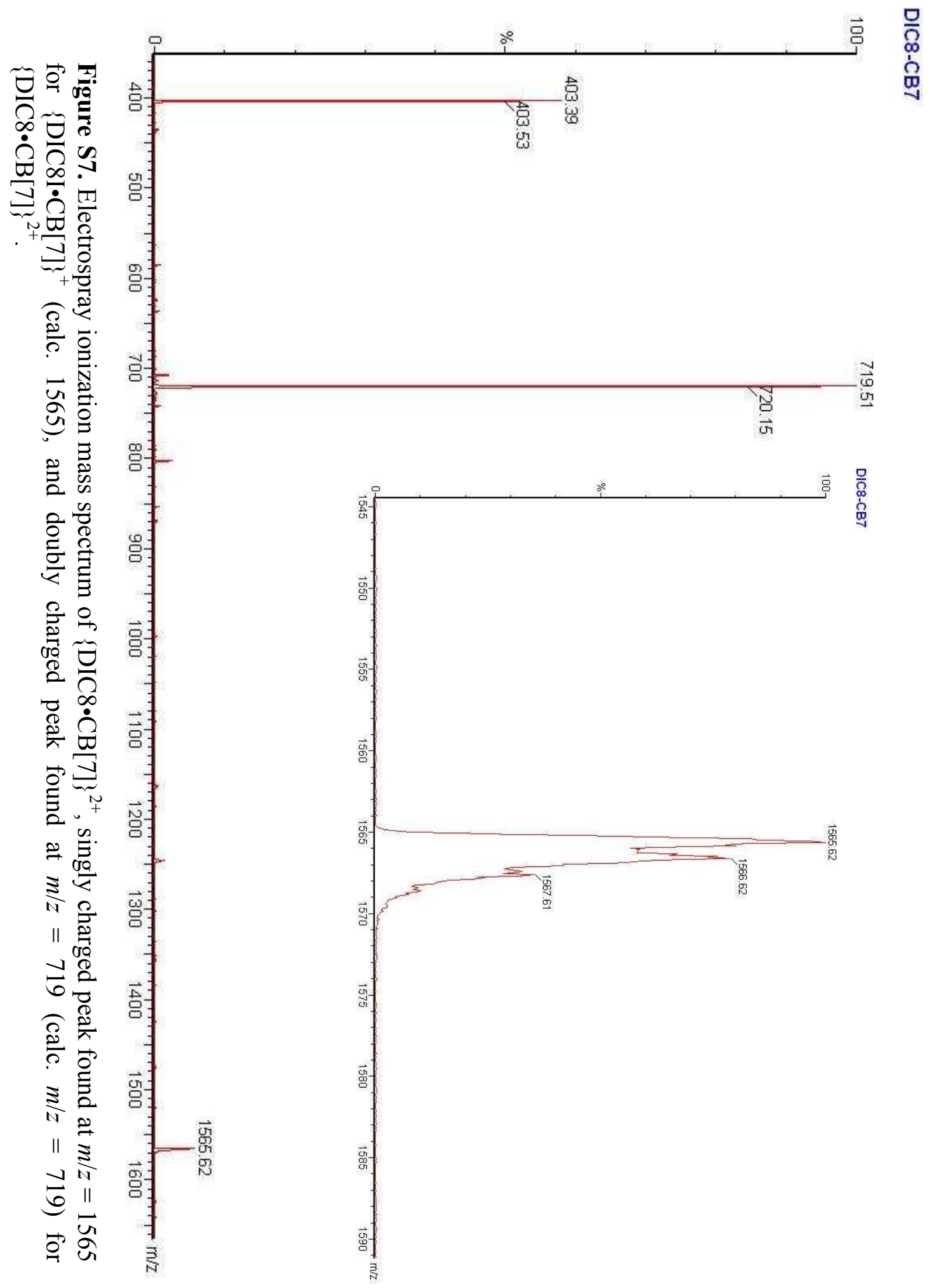




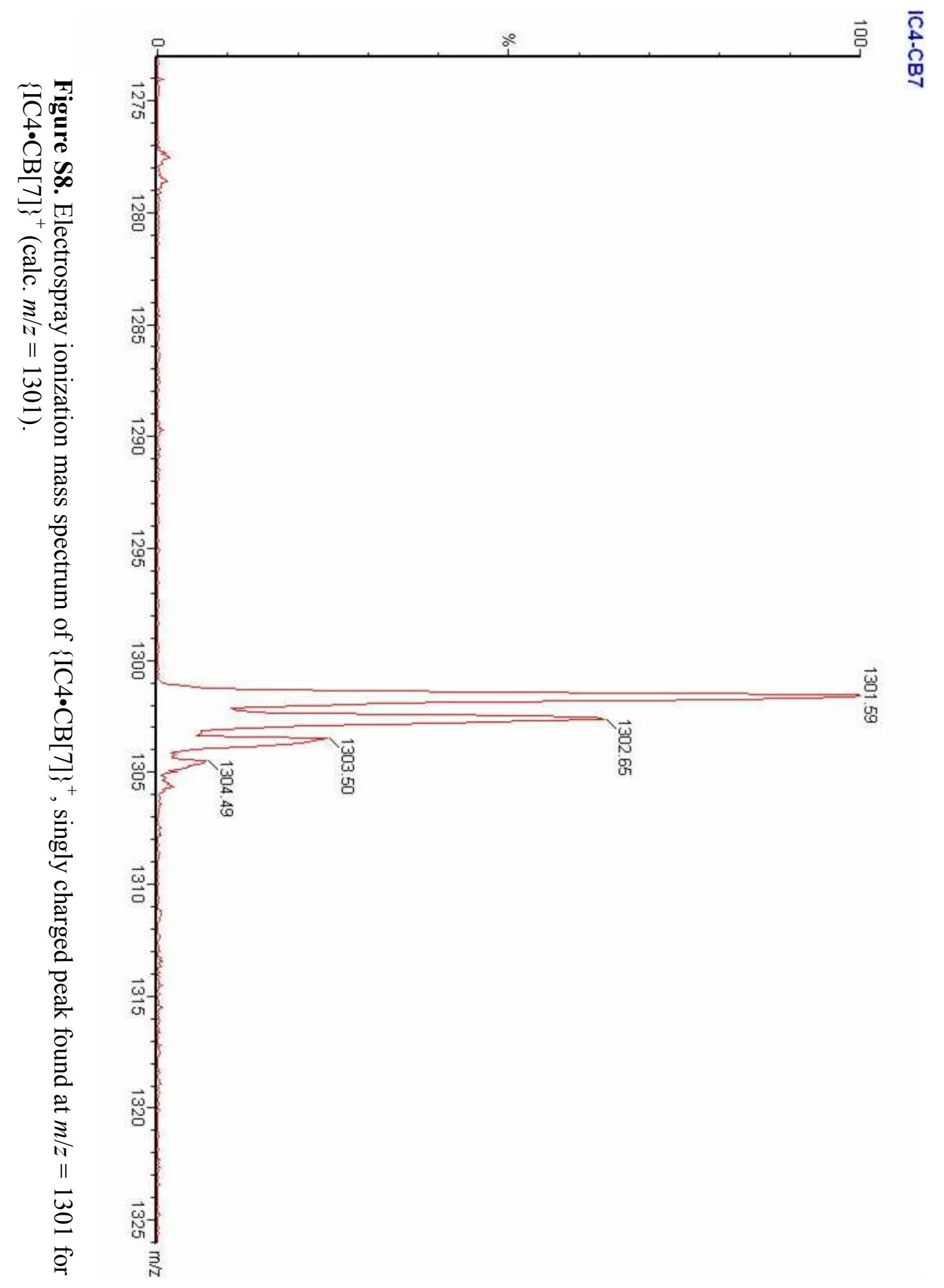




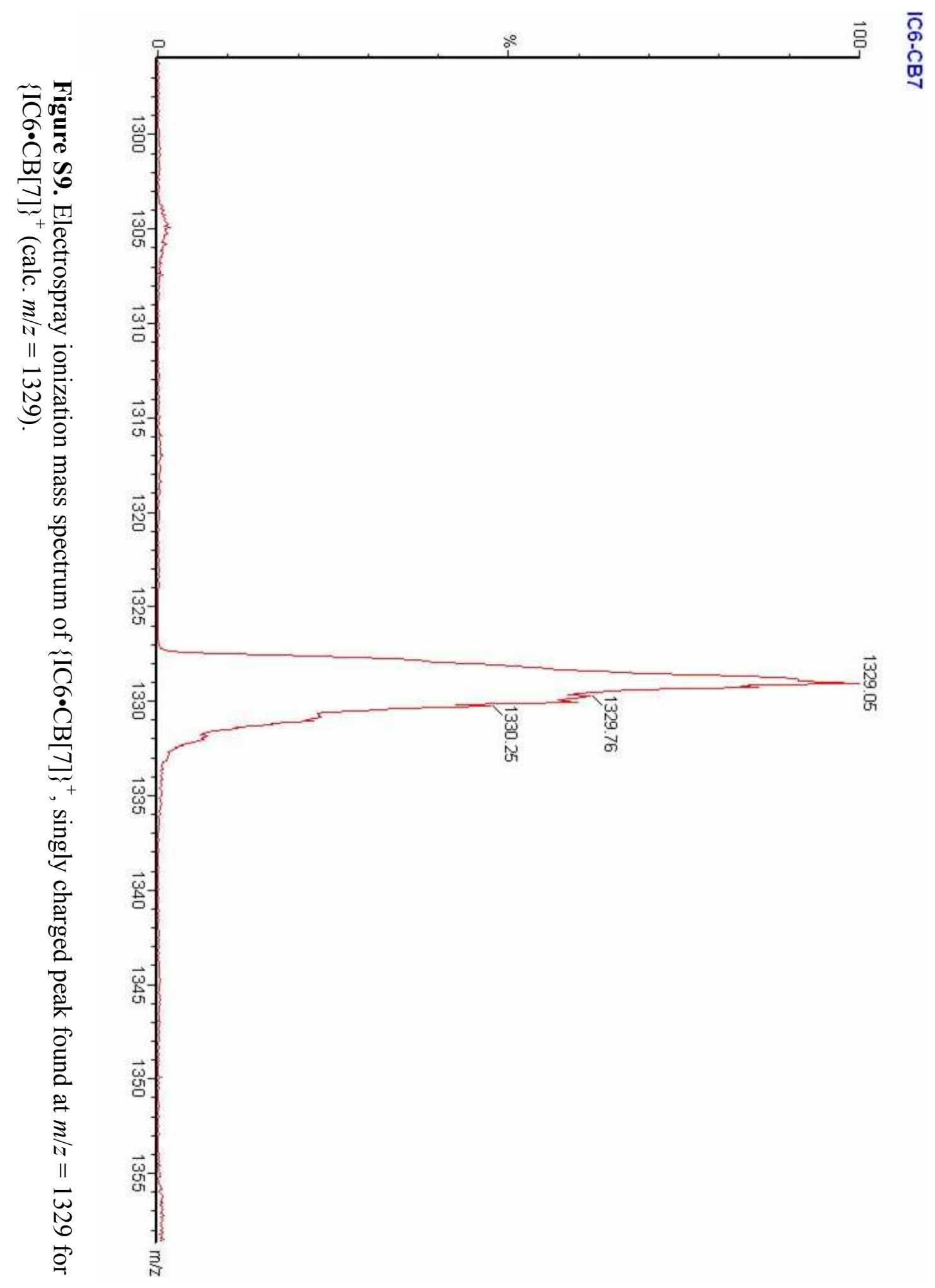




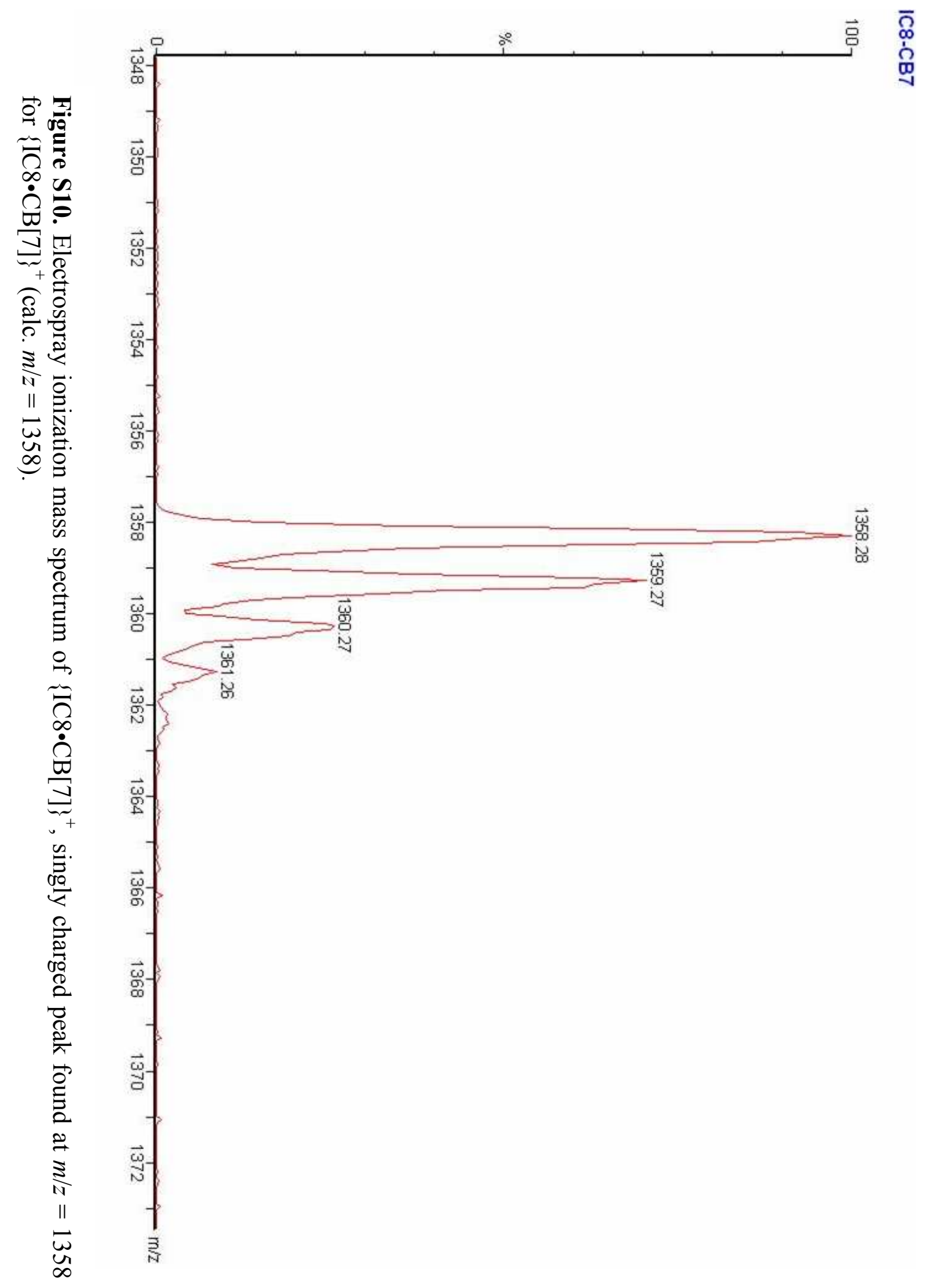



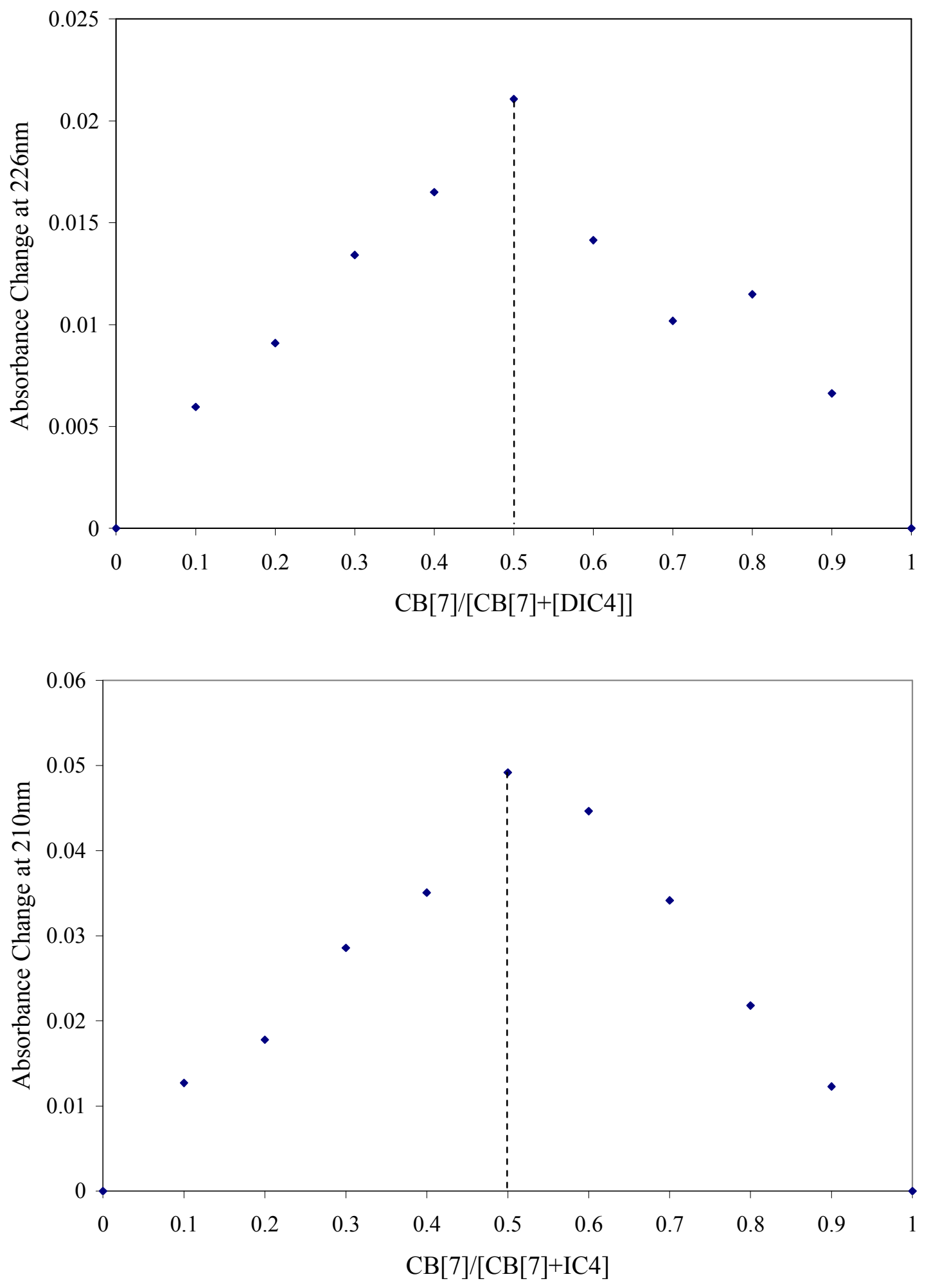

Figure S11. Job plots for DIC4 $4^{2+}$ and $\mathrm{IC}^{+}$. 

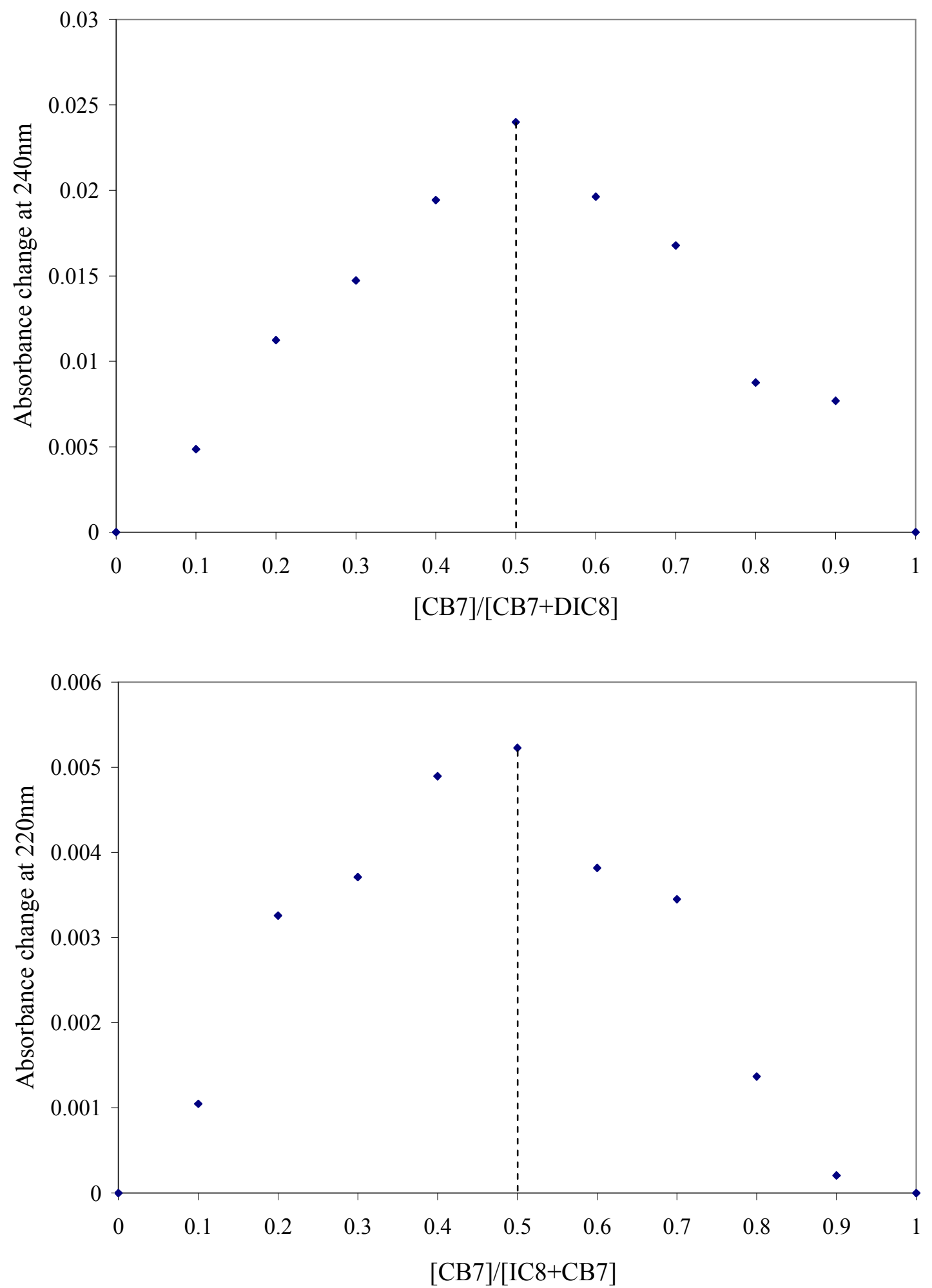

Figure S12. Job plots for DIC $8^{2+}$ and $\mathrm{IC} 8^{+}$. 


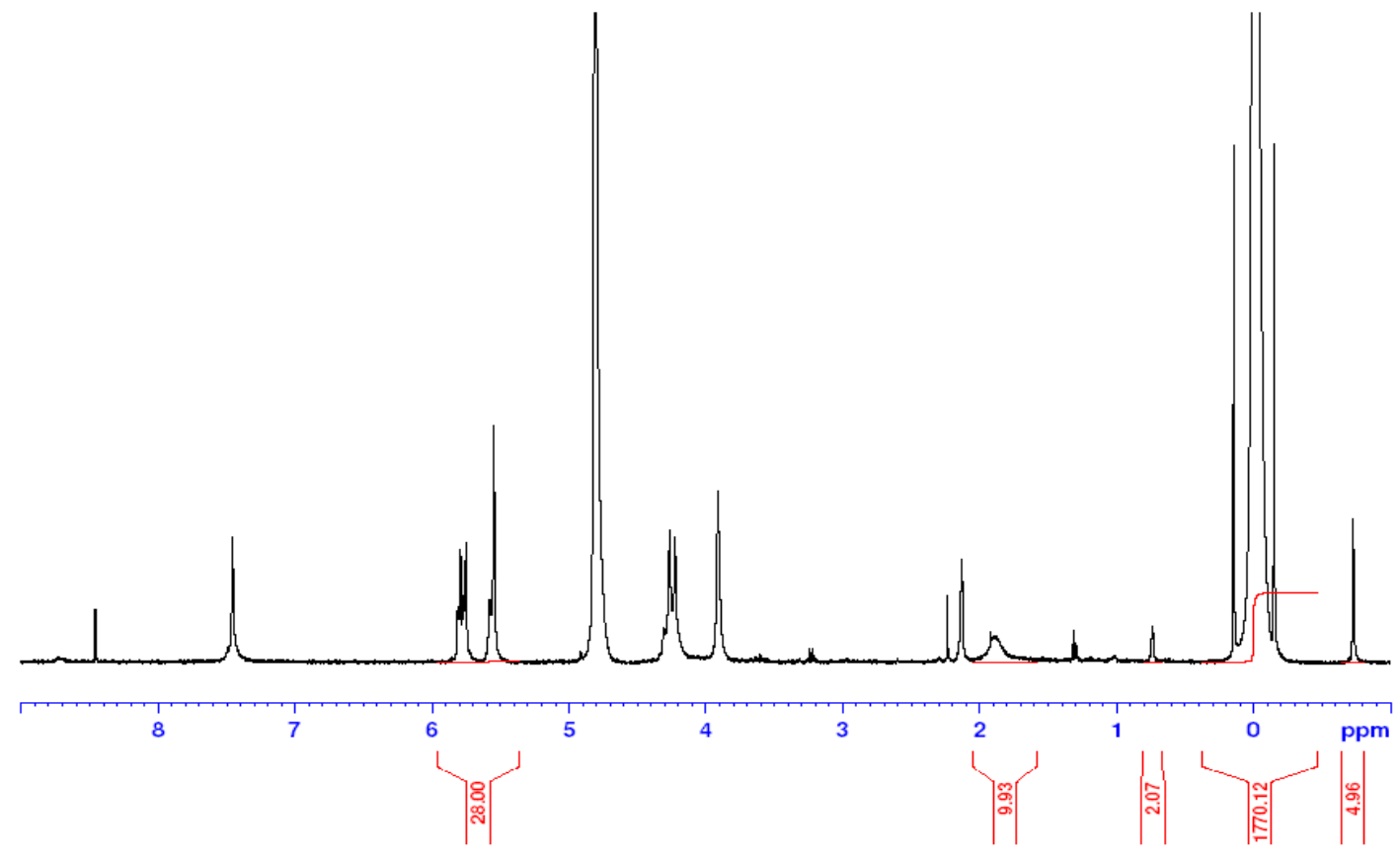

Figure S13. The ${ }^{1} \mathrm{H}$ NMR spectrum of competitive titration for $\mathrm{DIC}^{2+}$ upon complexation with $\mathrm{CB}[7]$ in the presence of TMSP.

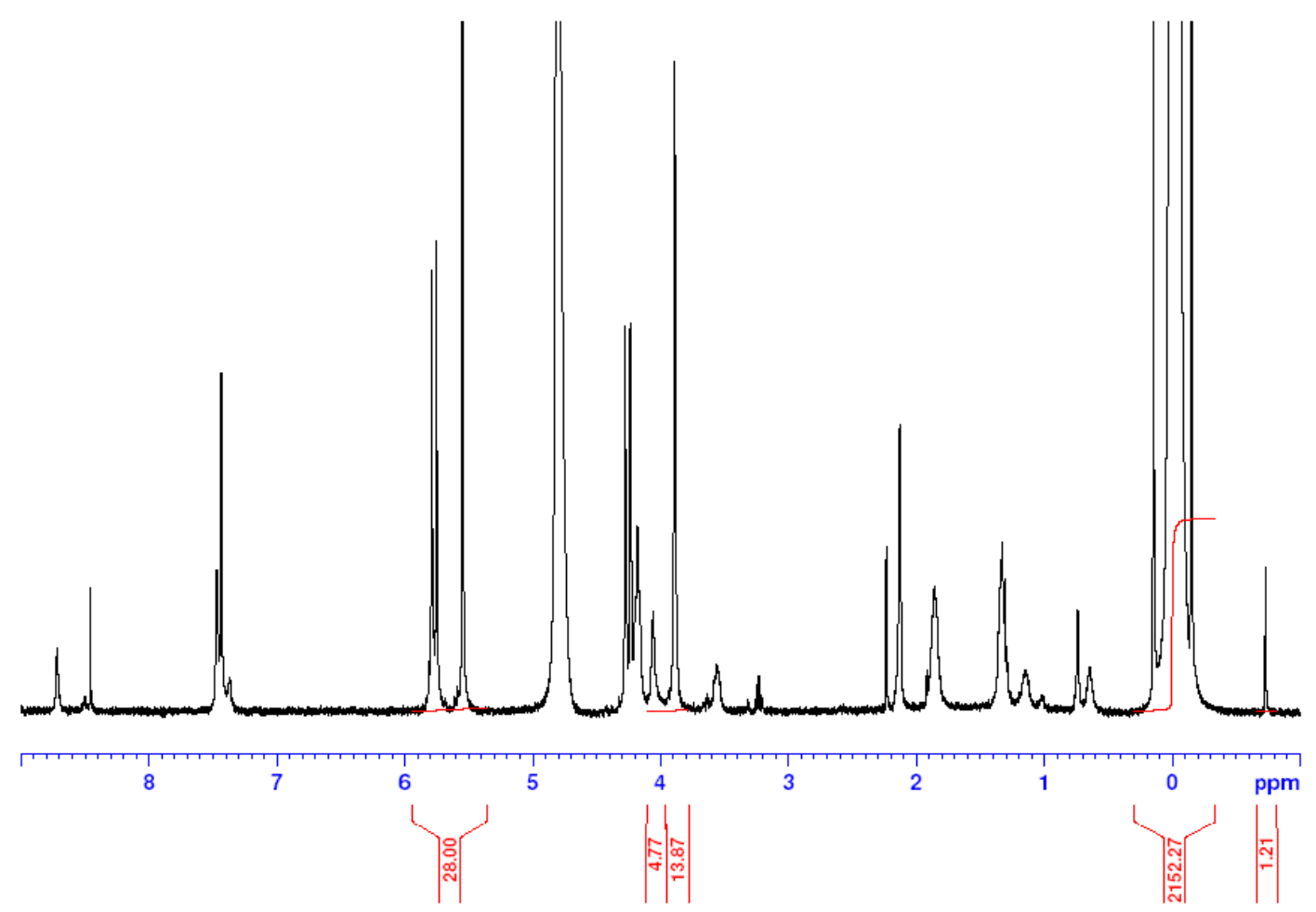

Figure S14. The ${ }^{1} \mathrm{H}$ NMR spectrum of competitive titration for $\mathrm{DIC6}^{2+}$ upon complexation with $\mathrm{CB}[7]$ in the presence of TMSP. 


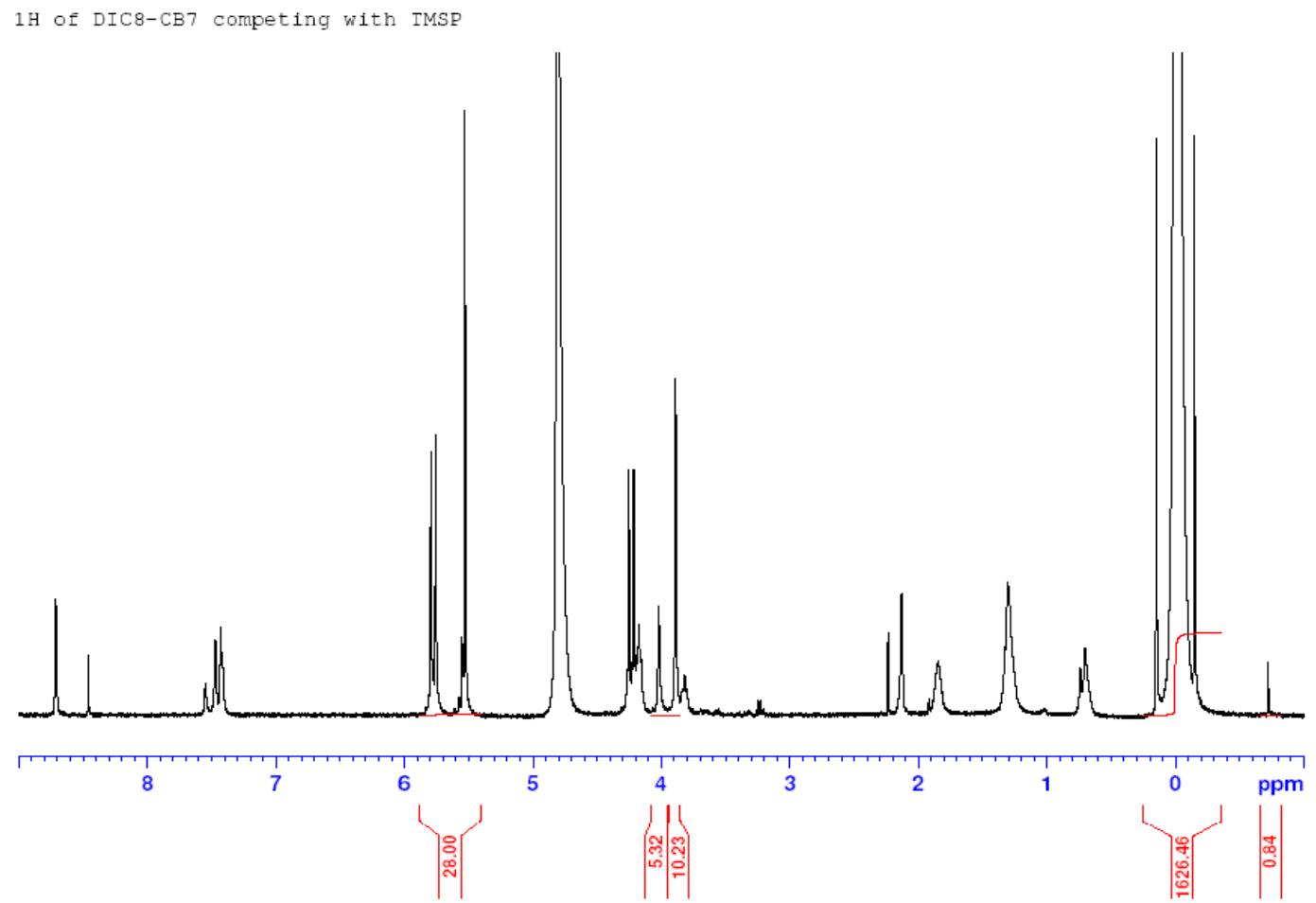

Figure S15. The ${ }^{1} \mathrm{H}$ NMR spectrum of competitive titration for $\mathrm{DIC}^{2+}$ upon complexation with $\mathrm{CB}[7]$ in the presence of TMSP.

$1 \mathrm{H}$ of $\mathrm{I} 4-\mathrm{CB} 7$ competing with TMSP

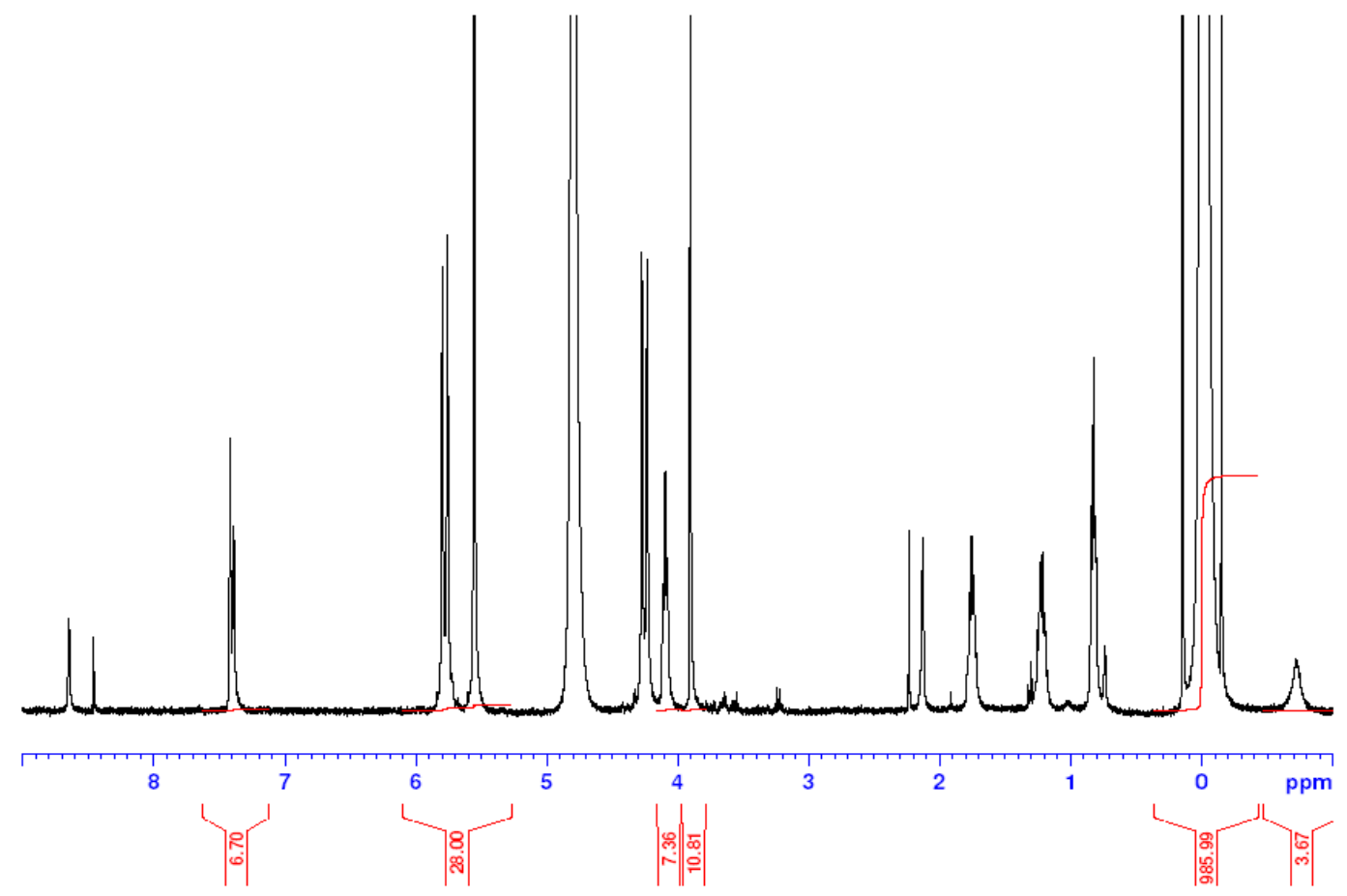

Figure S16. The ${ }^{1} \mathrm{H}$ NMR spectrum of competitive titration for $\mathrm{IC}^{+}$upon complexation with $\mathrm{CB}[7]$ in the presence of TMSP. 


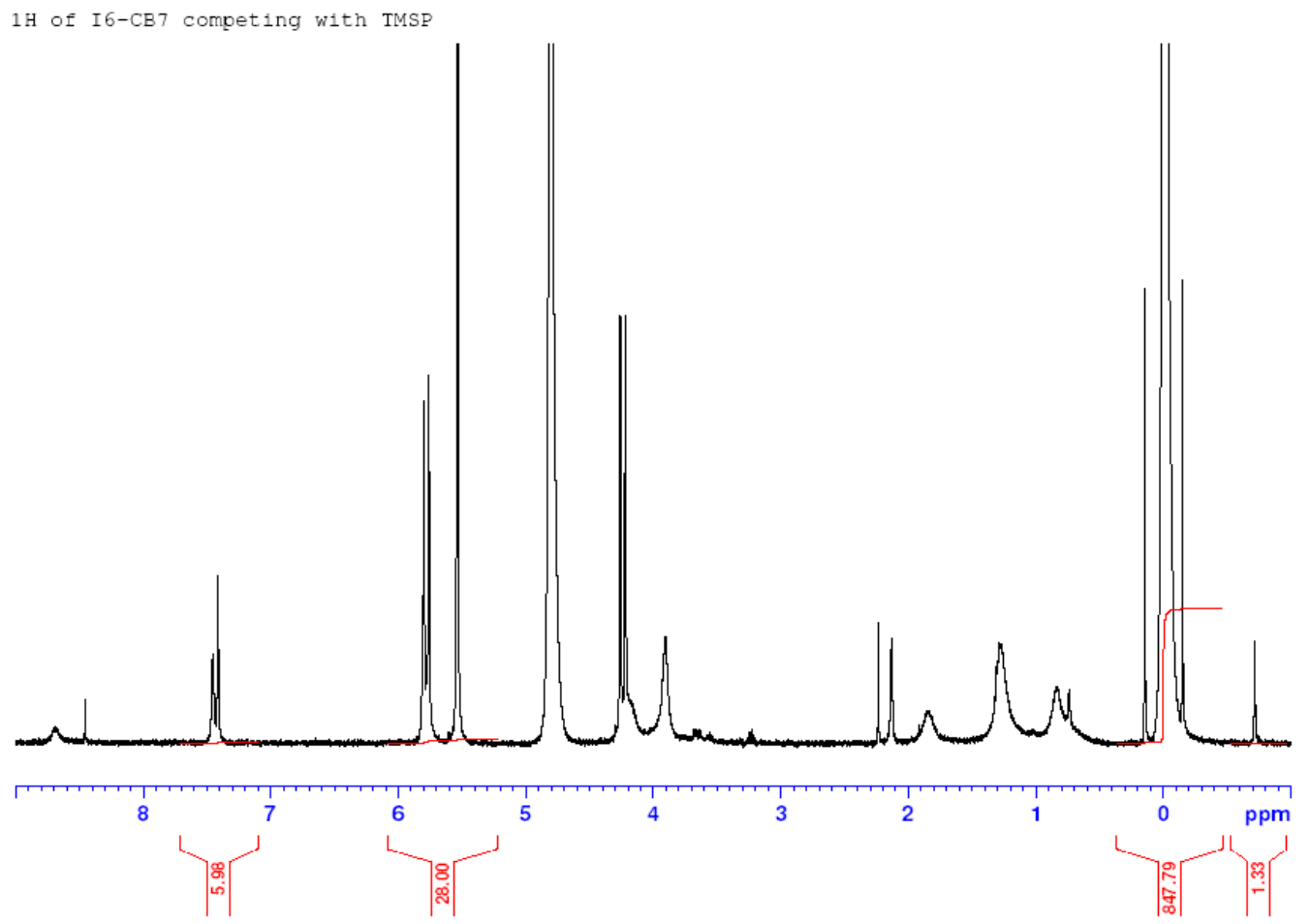

Figure S17. The ${ }^{1} \mathrm{H}$ NMR spectrum of competitive titration for $\mathrm{IC}^{+}{ }^{+}$upon complexation with $\mathrm{CB}[7]$ in the presence of TMSP.

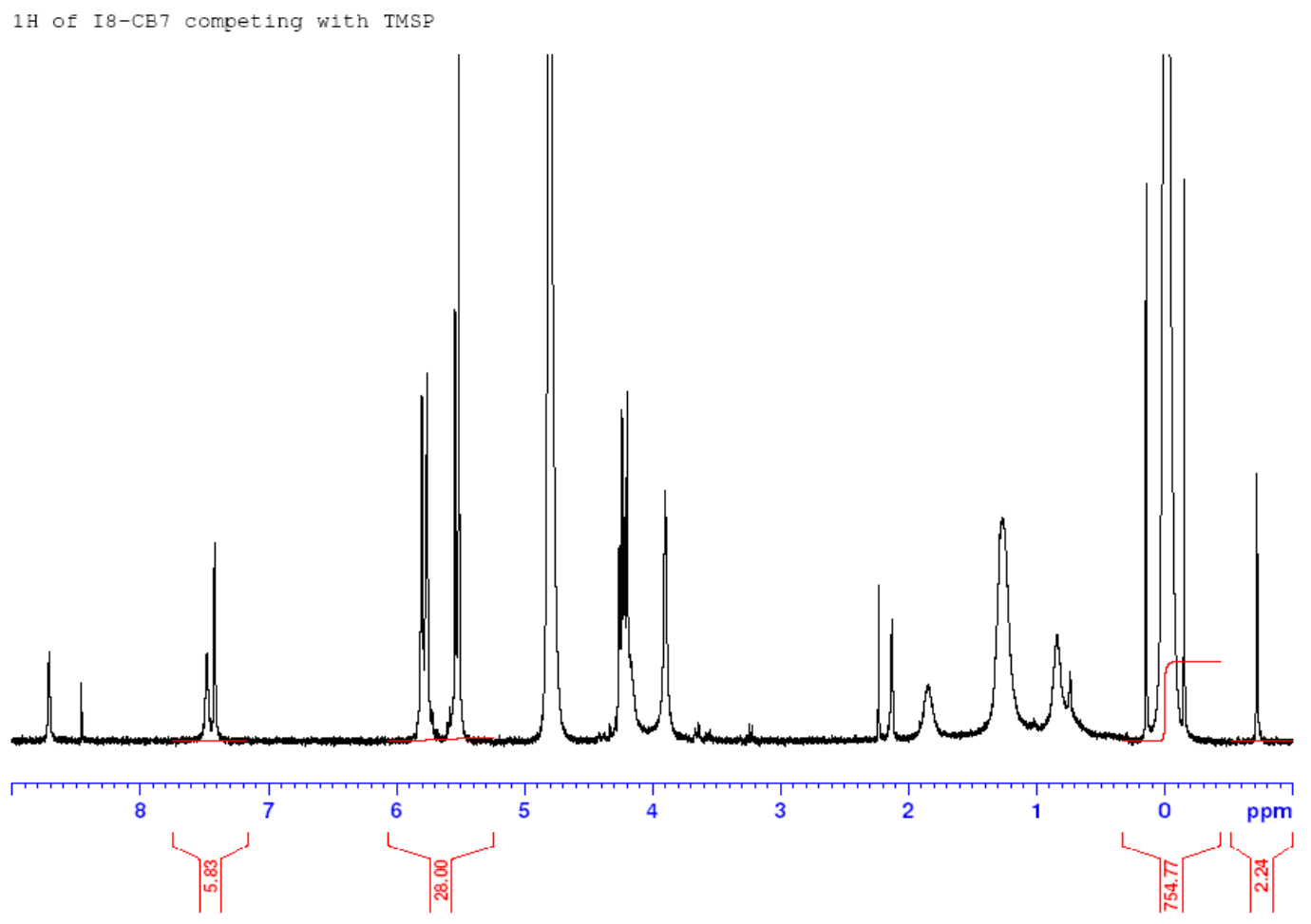

Figure S18. The ${ }^{1} \mathrm{H}$ NMR spectrum of competitive titration for $\mathrm{IC}^{+}$upon complexation with $\mathrm{CB}[7]$ in the presence of TMSP. 


\section{Determinations of Guest-Host Binding Constant:}

Trimethylsilylpropionic acid (deuterated TMSP) was used as the competitor successfully for all ionic ligands cations in the presence of limited CB[7]. TMSP has a binding constant with $\mathrm{CB}[7]$ of $(1.82 \pm 0.22) \times 10^{7} \mathrm{M}^{-1}{ }^{[1]}$ TMSP and imidazolium cations were added into a limiting quantity of $\mathrm{CB}[7](1 \mathrm{mM})$ in $\mathrm{D}_{2} \mathrm{O}$, please note that all concentrations were recalculated and confirmed based on ${ }^{1} \mathrm{H}$ integration. The concentrations of the free and bound guests were determined by integrations of their respective proton resonances.

\section{1) Binding constant of $\{\mathrm{IC} 8 \cdot \mathrm{CB}[7]\}^{+}$}

$[\mathrm{TMSP}]_{\text {bound }}=2.24 / 9=0.25 \mathrm{mM}$

$[\mathrm{TMSP}]_{\text {free }}=754.77 / 9=83.86 \mathrm{mM}$;

$[\mathrm{IC} 8]_{\mathrm{total}}=5.83 / 2=2.92 \mathrm{mM}$;

$[\mathrm{CB}[7]]_{\text {total }}=1 \mathrm{mM}$;

Therefore, $[\mathrm{IC} 8]_{\text {bound }}=[\mathrm{CB}[7]]_{\mathrm{total}}-[\mathrm{TMSP}]_{\text {bound }}=1-0.25=0.75 \mathrm{mM}$;

$[\mathrm{IC} 8]_{\text {free }}=2.92-0.75 \mathrm{mM}=2.17 \mathrm{mM}$;

As $\quad K_{\mathrm{IC} 8 \cdot \mathrm{CB}[7]} / K_{\mathrm{TMSP} \cdot \mathrm{CB}[7]}=[\mathrm{IC} 8 \cdot \mathrm{CB}[7]]_{\text {complex }} \times[\mathrm{TMSP}]_{\text {free }} /\left([\mathrm{TMSP} \cdot \mathrm{CB}[7]]_{\text {complex }} \times\right.$ $\left.[\mathrm{IC} 8]_{\text {free }}\right)$

$=0.75 \times 83.86 /(0.25 \times 2.17)=115.94$ and $K_{\mathrm{TMSP} \cdot \mathrm{CB}[7]}=(1.82 \pm 0.22) \times 10^{7} \mathrm{M}^{-1}$;

Therefore, $K_{\mathrm{IC} 8 \cdot \mathrm{CB}[7]}=(2.11 \pm 0.25) \times 10^{9} \mathrm{M}^{-1}$.

\section{2) Binding constant of $\{\mathrm{IC} 6 \bullet \mathrm{CB}[7]\}^{+}$}

$[\mathrm{TMSP}]_{\text {bound }}=1.33 / 9=0.15 \mathrm{mM}$

$[\mathrm{TMSP}]_{\text {free }}=847.79 / 9=94.20 \mathrm{mM}$; 
$[\mathrm{IC} 6]_{\text {total }}=5.98 / 2=2.99 \mathrm{mM}$;

$[\mathrm{CB} 7]_{\mathrm{total}}=1 \mathrm{mM}$;

Therefore, $[\text { IC6 }]_{\text {bound }}=[\mathrm{CB} 7]_{\mathrm{total}}-[\mathrm{TMSP}]_{\mathrm{bound}}=1-0.15=0.85 \mathrm{mM}$;

$[\text { IC6 }]_{\text {free }}=2.99-0.85=2.14 \mathrm{mM}$;

As $K_{\mathrm{IC} 6 \cdot \mathrm{CB}[7]} / K_{\mathrm{TMSP} \cdot \mathrm{CB}[7]}=[\mathrm{IC} 6 \cdot \mathrm{CB}[7]]_{\text {complex }} \times[\mathrm{TMSP}]_{\text {free }} /\left([\mathrm{TMSP} \cdot \mathrm{CB}[7]]_{\text {complex }} \times\right.$ $\left.[\mathrm{IC} 6]_{\text {free }}\right)$

$=0.85 \times 94.20 /(0.15 \times 2.14)=249.44$ and $K_{\mathrm{TMSP} \cdot \mathrm{CB}[7]}=(1.82 \pm 0.22) \times 10^{7} \mathrm{M}^{-1}$;

Therefore, $K_{\mathrm{IC} 6 \cdot \mathrm{CB}[7]}=(4.54 \pm 0.55) \times 10^{9} \mathrm{M}^{-1}$.

\section{3) Binding constant of $\{\mathrm{IC} 4 \cdot \mathrm{CB}[7]\}^{+}$}

$[\mathrm{TMSP}]_{\mathrm{bound}}=3.67 / 9=0.41 \mathrm{mM}$;

$[\text { TMSP }]_{\text {free }}=985.99 / 9=109.55 \mathrm{mM}$;

$[\mathrm{IC} 4]_{\mathrm{total}}=6.70 / 2=3.35 \mathrm{mM}$;

$[\mathrm{CB} 7]_{\mathrm{total}}=1 \mathrm{mM}$;

Therefore, $[\mathrm{IC} 4]_{\text {bound }}=[\mathrm{CB} 7]_{\text {total }}-[\mathrm{TMSP}]_{\mathrm{bound}}=1-0.41=0.59 \mathrm{mM}$;

$[\mathrm{IC} 4]_{\text {free }}=3.35-0.59=2.76 \mathrm{mM}$;

As $K_{\mathrm{IC} 4 \cdot \mathrm{CB}[7]} / K_{\mathrm{TMSP} \cdot \mathrm{CB}[7]}=[\mathrm{IC} 4 \cdot \mathrm{CB}[7]]_{\text {complex }} \times[\mathrm{TMSP}]_{\text {free }} /\left([\mathrm{TMSP} \cdot \mathrm{CB}[7]]_{\text {complex }} \times\right.$ $\left.[\mathrm{IC} 4]_{\text {free }}\right)$

$=0.59 \times 109.55 /(0.41 \times 2.76)=57.12$ and $K_{\mathrm{TMSP} \cdot \mathrm{CB}[7]}=(1.82 \pm 0.22) \times 10^{7} \mathrm{M}^{-1}$;

Therefore, $\mathrm{K}_{\mathrm{IC} 4 \cdot \mathrm{CB}[7]}=(1.04 \pm 0.13) \times 10^{9} \mathrm{M}^{-1}$.

\section{4) Binding constant of $\{\mathrm{DIC} \cdot \mathrm{CB}[7]\}^{2+}$}

$[\mathrm{TMSP}]_{\mathrm{bound}}=0.84 / 9=0.09 \mathrm{mM}$; 
$[\text { TMSP }]_{\text {free }}=1626.46 / 9=180.72 \mathrm{mM}$;

$[\mathrm{CB} 7]_{\mathrm{total}}=1 \mathrm{mM}$;

$[\mathrm{DIC} 8]_{\mathrm{bound}}=5.32 / 6=0.87 \mathrm{mM}$;

$[\mathrm{DIC} 8]_{\text {free }}=10.23 / 6=1.71 \mathrm{mM}$

As $K_{\mathrm{DIC} 8 \cdot \mathrm{CB}[7]} / K_{\mathrm{TMSP} \cdot \mathrm{CB}[7]}=[\mathrm{DIC} 8 \cdot \mathrm{CB}[7]]_{\text {complex }} \times[\mathrm{TMSP}]_{\text {free }} /\left([\mathrm{TMSP} \bullet \mathrm{CB}[7]]_{\text {complex }} \times\right.$ $\left.[\mathrm{DIC} 8]_{\text {free }}\right)$

$=0.87 \times 180.72 /(0.09 \times 1.71)=1021.6$ and $K_{\mathrm{TMSP} \cdot \mathrm{CB}[7]}=(1.82 \pm 0.22) \times 10^{7} \mathrm{M}^{-1}$;

Therefore, $K_{\mathrm{DIC} 8 \cdot \mathrm{CB}[7]}=(1.86 \pm 0.23) \times 10^{10} \mathrm{M}^{-1}$.

\section{5) Binding constant of $\left\{\mathrm{DIC} 6 \bullet^{\circ} \mathrm{CB}[7]\right\}^{2+}$}

$[\mathrm{TMSP}]_{\mathrm{bound}}=1.21 / 9=0.13 \mathrm{mM}$;

$[\mathrm{TMSP}]_{\text {free }}=2152.27 / 9=239.14 \mathrm{mM}$;

$[\mathrm{CB} 7]_{\mathrm{total}}=1 \mathrm{mM}$;

$[\text { DIC6 }]_{\text {free }}=13.87 / 6=2.31 \mathrm{mM}$

$[\mathrm{DIC} 6]_{\mathrm{bound}}=4.77 / 6=0.80 \mathrm{mM}$;

As $K_{\mathrm{DIC} 6 \cdot \mathrm{CB}[7]} / K_{\mathrm{TMSP} \cdot \mathrm{CB}[7]}=[\mathrm{DIC} 8 \cdot \mathrm{CB}[7]]_{\text {complex }} \times[\mathrm{TMSP}]_{\text {free }} /\left([\mathrm{TMSP} \cdot \mathrm{CB}[7]]_{\mathrm{complex}} \times\right.$ $\left.[\mathrm{DIC6}]_{\text {free }}\right)$

$=0.80 \times 239.14 /(0.13 \times 2.31)=637.1$ and $K_{\mathrm{TMSP} \cdot \mathrm{CB}[7]}=(1.82 \pm 0.22) \times 10^{7} \mathrm{M}^{-1}$;

Therefore, $K_{\mathrm{DIC} 6 \cdot \mathrm{CB}[7]}=(1.16 \pm 0.14) \times 10^{10} \mathrm{M}^{-1}$.

\section{6) Binding constant of $\{\mathrm{DIC} 4 \cdot \mathrm{CB}[7]\}^{2+}$}

$[\mathrm{TMSP}]_{\mathrm{bound}}=4.96 / 9=0.55 \mathrm{mM}$;

$[\mathrm{TMSP}]_{\text {free }}=1770.12 / 9=196.68 \mathrm{mM}$;

$[\mathrm{CB} 7]_{\text {total }}=1 \mathrm{mM}$; limiting reagent

$[\mathrm{DIC} 4]_{\mathrm{free}}=9.93 / 4=2.48 \mathrm{mM}$ 
$[\mathrm{DIC} 4]_{\mathrm{bound}}=2.07 / 4=0.52 \mathrm{mM}$;

As $K_{\mathrm{DIC} 4 \cdot \mathrm{CB}[7]} / K_{\mathrm{TMSP} \cdot \mathrm{CB}[7]}=[\mathrm{DIC} 4 \cdot \mathrm{CB}[7]]_{\mathrm{complex}} \times[\mathrm{TMSP}]_{\mathrm{free}} /\left([\mathrm{TMSP} \cdot \mathrm{CB}[7]]_{\mathrm{complex}} \times\right.$ $\left.[\mathrm{DIC} 4]_{\text {free }}\right)$

$=0.52 \times 196.68 /(0.55 \times 2.48)=75.1$ and $K_{\mathrm{TMSP} \cdot \mathrm{CB}[7]}=(1.82 \pm 0.22) \times 10^{7} \mathrm{M}^{-1}$;

Therefore, $K_{\mathrm{DIC} 4 \cdot \mathrm{CB}[7]}=(1.37 \pm 0.17) \times 10^{9} \mathrm{M}^{-1}$.

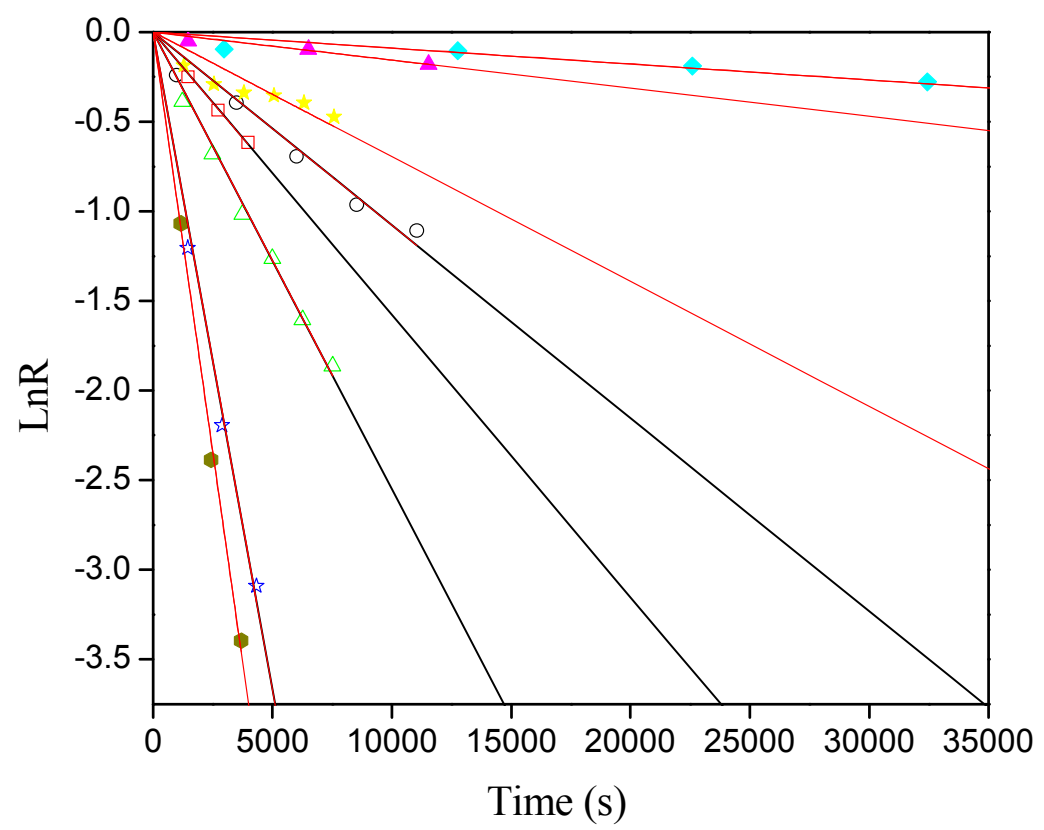

Figure S19. Semilogarithmic plot of $\ln R$ against time for the $\mathrm{C}(2)$-proton deuterium exchange of $\mathrm{DIC}^{2+}(2 \mathrm{mM})$ at $\mathrm{p} D=8.04(\circ), 8.20(\square), 8.44(\triangle)$, and 8.90 (放), and of $\{\mathrm{DIC} 4 \cdot \mathrm{CB}[7]\}^{2+}(2 \mathrm{mM})$ at $\mathrm{p} D=7.85(\varpi), 7.96(\Delta), 8.48(\star)$ and $9.55(\bullet)$. 


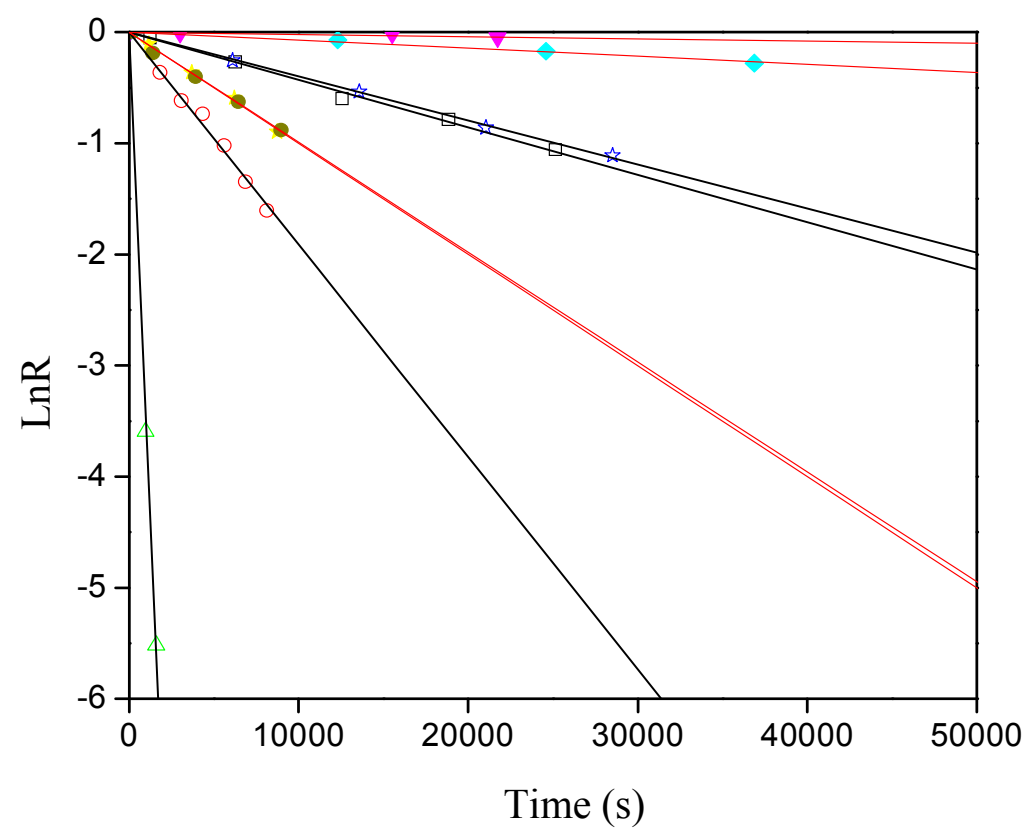

Figure S20. Semilogarithmic plot of $\ln R$ against time for the $\mathrm{C}(2)$-proton deuterium exchange of $\mathrm{DIC}^{2+}(2 \mathrm{mM})$ at $\mathrm{p} D=7.83$ (污), 7.94 (口), $8.53(\circ)$, and $9.64(\triangle)$, and of $\{\mathrm{DIC} 6 \bullet \mathrm{CB}[7]\}^{2+}(2 \mathrm{mM})$ at $\mathrm{p} D=8.09(\Delta), 8.53(\square), 8.87(\star)$ and $9.53(\bullet)$.

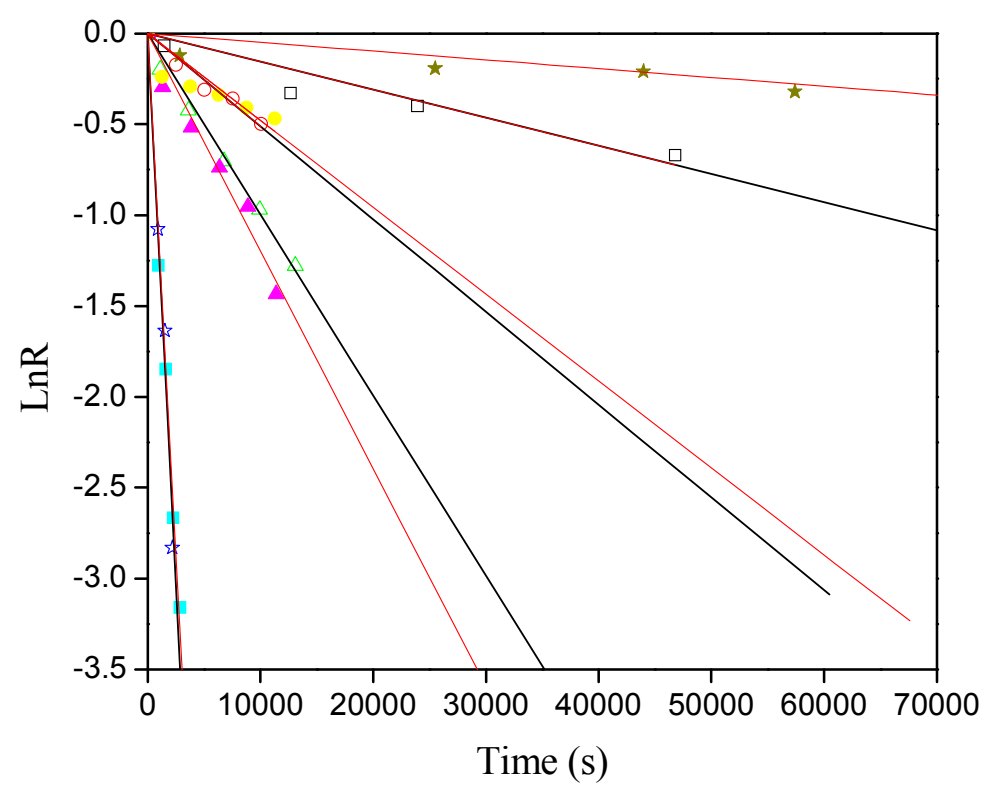

Figure S21. Semilogarithmic plot of $\ln R$ against time for the $\mathrm{C}(2)$-proton deuterium exchange of $\mathrm{DIC}^{2+}(2 \mathrm{mM})$ at $\mathrm{p} D=7.28(\square), 8.00(\circ), 8.25(\Delta)$, and 9.22 ( 5 ), and of $\{\mathrm{DIC} 8 \cdot \mathrm{CB}[7]\}^{2+}(2 \mathrm{mM})$ at $\mathrm{p} D=7.94(\star), 8.20(\circ), 8.90(\Delta)$ and $10.33(\square)$. 


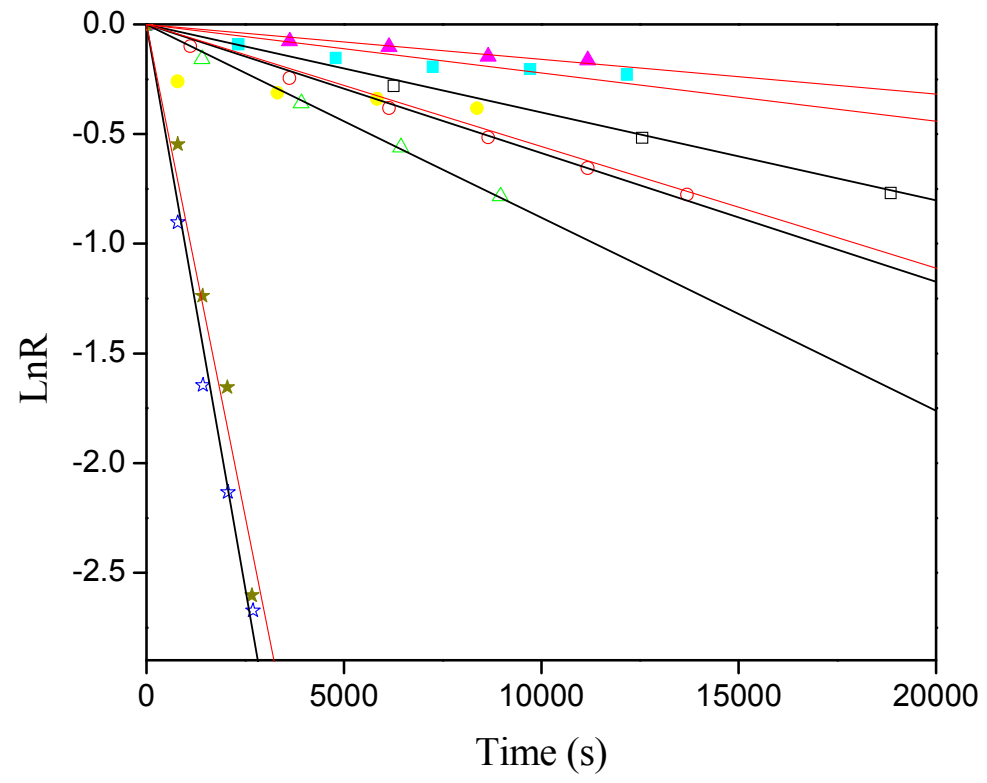

Figure S22. Semilogarithmic plot of $\ln R$ against time for the $\mathrm{C}(2)$-proton deuterium exchange of $\mathrm{IC}^{+}(2 \mathrm{mM})$ at $\mathrm{p} D=8.08(\square), 8.25(\circ), 8.43(\triangle)$, and $9.38($ 㧒), and of $\{\mathrm{IC} 4 \cdot \mathrm{CB}[7]\}^{+}(2 \mathrm{mM})$ at $\mathrm{p} D=7.72(\triangle), 7.97(\Xi), 8.14(\bullet)$ and $9.69(\star)$.

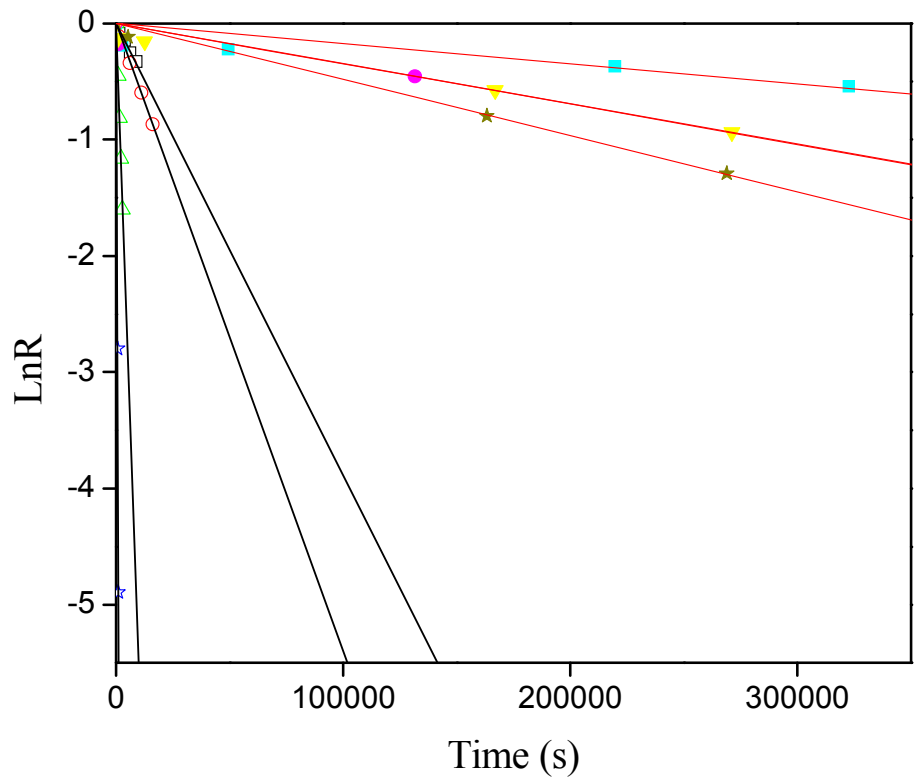

Figure S23. Semilogarithmic plot of $\ln R$ against time for the $\mathrm{C}(2)$-proton deuterium exchange of IC6 ${ }^{+}(2 \mathrm{mM})$ at $\mathrm{p} D=8.07(\square), 8.25(\circ), 9.02(\Delta)$, and $9.88(\succsim)$, and of $\{\mathrm{IC} 6 \cdot \mathrm{CB}[7]\}^{+}(2 \mathrm{mM})$ at $\mathrm{p} D=7.77(\backsim), 8.05(\bullet), 8.27(\triangle)$ and $8.48(\star)$. 


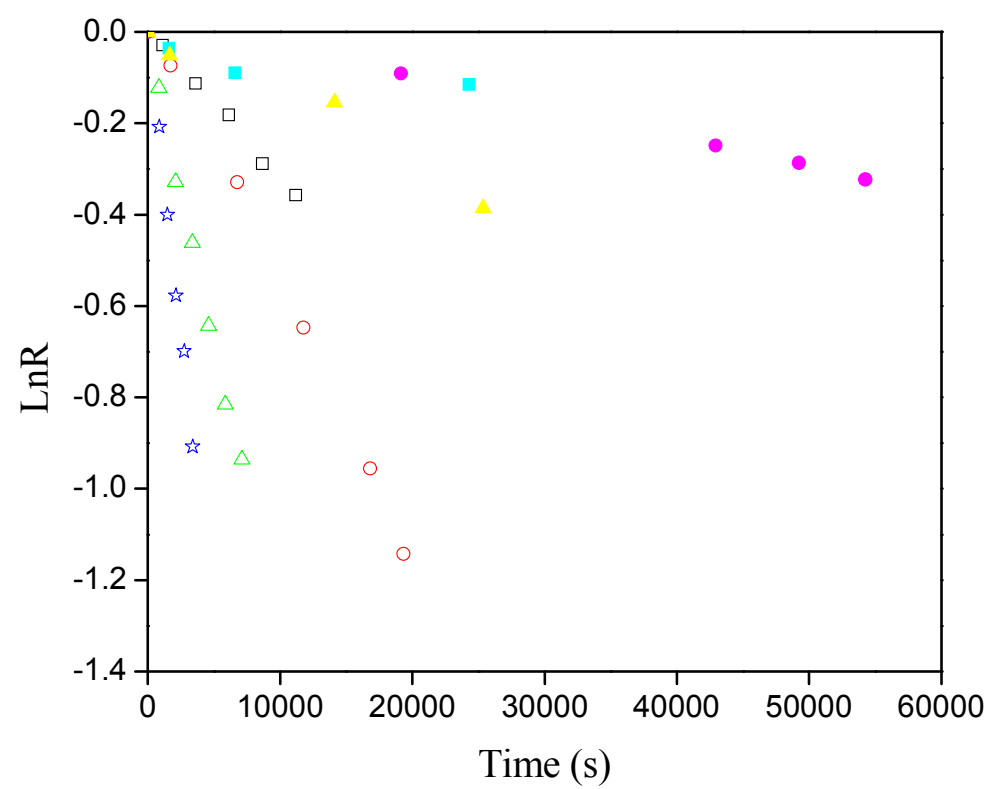

Figure S24. Semilogarithmic plot of $\ln R$ against time for the $\mathrm{C}(2)$-proton deuterium exchange for $\mathrm{IC}^{+}(2 \mathrm{mM})$ at $\mathrm{p} D=8.07(\square), 8.20(\circ), 8.54(\triangle)$, and 8.87 (场), and for $\{\mathrm{IC} 8 \cdot \mathrm{CB}[7]\}^{+}(2 \mathrm{mM})$ at $\mathrm{p} D=7.81(\square), 7.99(\bullet), 8.21(\triangle)$.

\section{Determination of the first-order rate constants by semilogarithmic plots}

The proton/deuterium exchange was monitored via ${ }^{1} \mathrm{H}$ NMR spectroscopy as described above. Values describing the reaction progress, $R$, could be calculated from the integrations of the of $\mathrm{C}(2)$-proton resonances $\left[\left(\mathrm{I}_{2 \mathrm{H}}\right)_{\mathrm{t}}\right]$ at time $t$, with an internal reference $\left[\left(\mathrm{I}_{2 \mathrm{H}}\right)_{0}\right]$, at the beginning of the experiment (or at $t=0$ ), according to equation (1).

$R=\left(\mathrm{I}_{2 \mathrm{H}}\right)_{t} /\left(\mathrm{I}_{2 \mathrm{H}}\right)_{0}$

$\ln R=-k_{\mathrm{ex}} t$

From equation (2), the first-order rate constant $k_{\mathrm{ex}}$ could be determined from the slope of the linear semilogarithmic plot of the reaction progress versus the reaction time. Therefore, the first-order rate constant $k_{\mathrm{ex}}$ values, in the presence and in the absence of $\mathrm{CB}[7]$, at various $\mathrm{p} D$ conditions could be determined. ${ }^{[2]}{ }^{[3]}$ 


\section{Determination of the second-order rate constants}

The second-order rate constant, $k_{\mathrm{DO}}$, was calculated from the $y$-axis intercept of the linear fit with a fixed slope of one by plotting $\log k_{\mathrm{ex}}$ (first-order rate constants) against the $\mathrm{p} D$ values according to the equation (3). ${ }^{[4]}$

$\log k_{\mathrm{ex}}=\log \left(k_{\mathrm{DO}} K_{\mathrm{W}} / \gamma_{\mathrm{OL}}\right)+\mathrm{p} D$

The value of $\mathrm{p} K_{\mathrm{W}}=14.87$ (for deuterated water). Under our experimental conditions the activity coefficient for deuterium oxide is $\gamma_{\mathrm{OL}}=0.83$, as estimated from the solvent conditions and the ionic strength. ${ }^{[4]}$

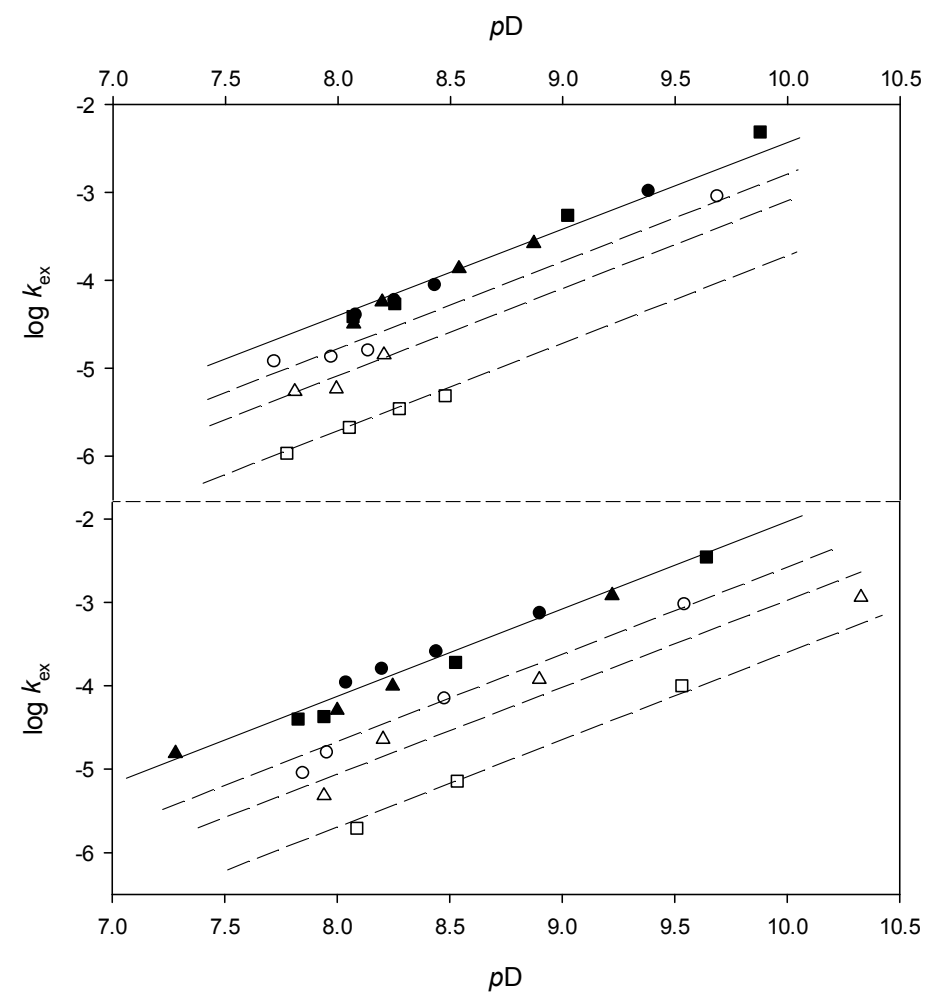

Figure S25. Plots of $\log k_{\text {ex }}$ against $\mathrm{p} D$ for the $\mathrm{C}(2)-\mathrm{H} / \mathrm{D}$ exchange reactions for the dications (lower portion): $\mathrm{DIC}^{2+}(\bullet)$ and $\{\mathrm{DIC} 4 \cdot \mathrm{CB}[7]\}^{2+}(0) ; \mathrm{DIC}^{2+}(\boldsymbol{\bullet})$ and $\left\{\text { DIC6 } \bullet^{\circ B}[7]\right\}^{2+}(\square)$; and $\operatorname{DIC}^{2+}(\boldsymbol{\Delta})$ and $\{\mathrm{DIC} 8 \cdot \mathrm{CB}[7]\}^{2+}(\Delta)$, and the monocations (upper portion): $\mathrm{IC}^{+}(\bullet), \operatorname{IC}^{+}(\bullet), \operatorname{IC}^{+}(\boldsymbol{\Delta}),\{\mathrm{IC} 4 \bullet \mathrm{CB}[7]\}^{+}(0)$, $\left\{\mathrm{IC} 6 \bullet^{\circ} \mathrm{CB}[7]\right\}^{+}(\square)$, and $\{\mathrm{IC} 8 \cdot \mathrm{CB}[7]\}^{+}(\Delta)$ in $\mathrm{D} 2 \mathrm{O}$. 

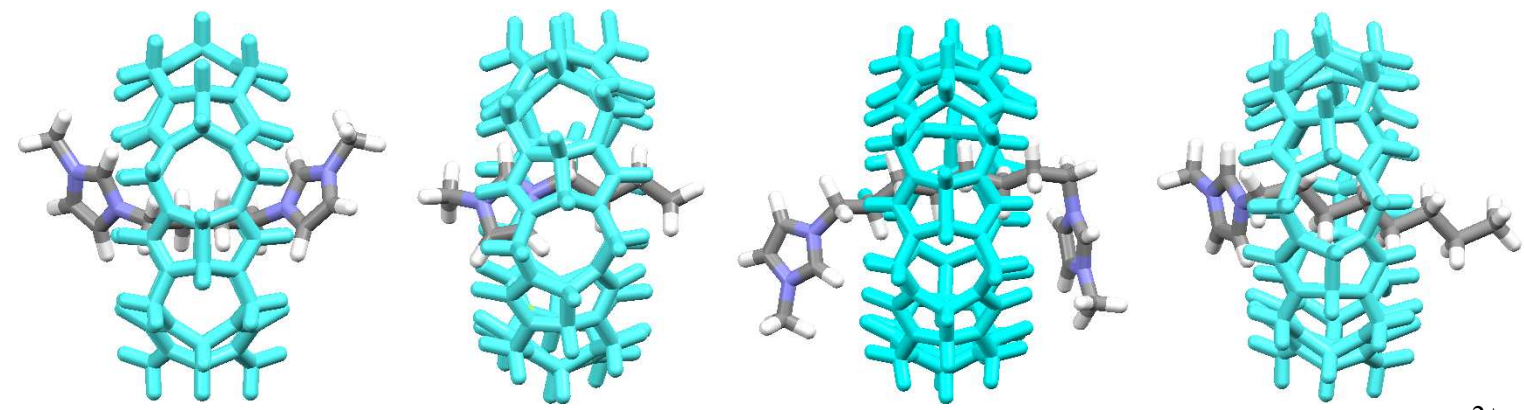

Figure S26. Energy-minimized structures $\left(\mathrm{HF} / 3-21 \mathrm{G}^{* *}\right.$ basis set $)$ of $\{\mathrm{DIC} 4 \cdot \mathrm{CB}[7]\}^{2+}$, $\{\mathrm{IC} 4 \cdot \mathrm{CB}[7]\}^{+},\{\mathrm{DIC} 8 \cdot \mathrm{CB}[7]\}^{2+}$ and $\{\mathrm{IC} 8 \bullet \mathrm{CB}[7]\}^{+}$(From left to right).

\section{The H-bonding length and angles}

$\{\mathrm{DIC} 4 \cdot \mathrm{CB}[7]\}^{2+}$ : angle: $147.0^{\circ}($ distance $2.22 \AA)$ on one end; $147.0^{\circ}(2.22 \AA), 119.5^{\circ}$

$(2.38 \AA)$ on the other;

$\left\{\mathrm{DIC} 6{ }^{\circ} \mathrm{CB}[7]\right\}^{2+}: 148.8^{\circ}(2.35 \AA)$ on one end; $140.1^{\circ}(2.72 \AA)$ on the other;

$\{\mathrm{DIC} 8 \cdot \mathrm{CB}[7]\}^{2+}: 119.2^{\circ}(3.76 \AA)$ on one end; $153.9^{\circ}(2.20 \AA), 114.3^{\circ}(2.42 \AA)$ on the

other end.

$\{\mathrm{IC} 4 \cdot \mathrm{CB}[7]\}^{+}: 154.3^{\circ}(2.19 \AA), 107.6^{\circ}(2.52 \AA)$

$\{\mathrm{IC} 6 \cdot \mathrm{CB}[7]\}^{+}: 142.3^{\circ}(2.26 \AA), 101.5^{\circ}(2.52 \AA)$

$\{\mathrm{IC} 8 \cdot \mathrm{CB}[7]\}^{+}: 144.7^{\circ}(2.21 \AA), 99.7^{\circ}(2.64 \AA)$.

\section{Full Citation for Reference 24}

(24) Frisch, M. J.; Trucks, G. W.; Schlegel, H. B.; Scuseria, G. E.; Robb, M. A.; Cheeseman, J. R.; Montgomery, J. A. Jr.; Vreven, T.; Kudin, N.; Burant, J. C.; Millam, J. M.; Iyengar, S. S.; Tomasi, J.; Barone, V.; Mennucci, B.; Cossi, M.; Scalmani, G.; Rega, N.; Petersson, G. A.; Nakatsuji, H.; Hada, M.; Ehara, M.; Toyota, K.; Fukuda, R.; Hasegawa, J.; Ishida, M.; Nakajima, T.; Honda, Y.; Kitao, O.; Nakai, H.; Klene, M.; Li, X.; Knox, J. E.; Hratchian, H. P.; Cross, J. B.; Adamo, C.; Jaramillo, J.; Gomperts, R.; Stratmann, R. E.; Yazyev, O.; Austin, A. J.; Cammi, R.; Pomelli, C.; Ochterski, J. W.; Ayala, P. Y.; Morokuma, K.; Voth, G. A.; Salvador, P.; Dannenberg, J. J.; Zakrzewski, V. G.; Dapprich, S.; Daniels, A. D.; Strain, M. C.; Farkas, O.; Malick, D. K.; Rabuck, A. D.; Raghavachari, K.; Foresman, J. B.; Ortiz, J. V.; Cui, Q.; Baboul, A. G.; Clifford, S.; 
Cioslowski, J.; Stefanov, B. B.; Liu, G.; Liashenko, A.; Piskorz, P.; Komaromi, I.; Martin, R. L.; Fox, D. J.; Keith, T.; Al-Laham, M. A.; Peng, C. Y.; Nanayakkara, A.; Challacombe, M.; Gill, P. M. W.; Johnson, B.; Chen, W.; Wong, M. W.; Gonzalez, C.; Pople, J. A. Gaussian 03, Revision C.02, Gaussian, Pittsburgh, PA 2004.

\section{Absolute energies (in Hartrees) and coordinates of the atoms of the $\mathrm{CB}$ [7]complexes}

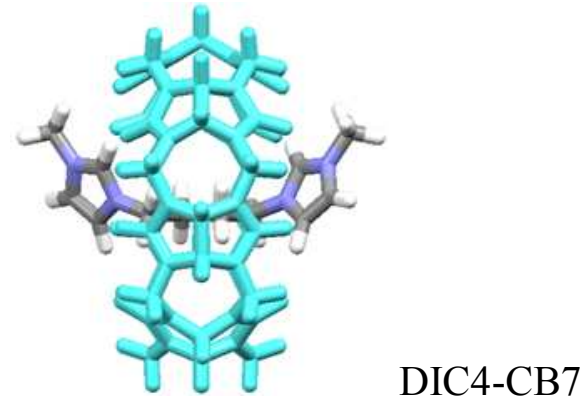

SCF Done: $E=-4844.64689327$

REMARK 1 File created by GaussView 5.0.8

$\begin{array}{llllllll}\text { HETATM } & 1 & \mathrm{C} & 0 & 5.755 & 1.814 & 0.168 & \mathrm{C} \\ \text { HETATM } & 2 & \mathrm{C} & 0 & 5.074 & 3.238 & 0.313 & \mathrm{C} \\ \text { HETATM } & 3 & \mathrm{~N} & 0 & 4.313 & 3.098 & 1.530 & \mathrm{~N} \\ \text { HETATM } & 4 & \mathrm{C} & 0 & 4.337 & 1.827 & 2.045 & \mathrm{C} \\ \text { HETATM } & 5 & \mathrm{~N} & 0 & 5.242 & 1.097 & 1.315 & \mathrm{~N} \\ \text { HETATM } & 6 & \mathrm{~N} & 0 & 5.253 & 1.354 & -1.108 & \mathrm{~N} \\ \text { HETATM } & 7 & \mathrm{C} & 0 & 4.345 & 2.209 & -1.675 & \mathrm{C} \\ \text { HETATM } & 8 & \mathrm{~N} & 0 & 4.279 & 3.336 & -0.896 & \mathrm{~N} \\ \text { HETATM } & 9 & \mathrm{O} & 0 & 3.719 & 1.434 & 3.012 & \mathrm{O} \\ \text { HETATM } & 10 & \mathrm{O} & 0 & 3.756 & 2.033 & -2.724 & \mathrm{O} \\ \text { HETATM } & 11 & \mathrm{C} & 0 & 2.163 & 5.447 & 0.536 & \mathrm{C} \\ \text { HETATM } & 12 & \mathrm{C} & 0 & 0.608 & 5.742 & 0.579 & \mathrm{C} \\ \text { HETATM } & 13 & \mathrm{~N} & 0 & 0.174 & 4.954 & 1.706 & \mathrm{~N} \\ \text { HETATM } & 14 & \mathrm{C} & 0 & 1.183 & 4.226 & 2.292 & \mathrm{C} \\ \text { HETATM } & 15 & \mathrm{~N} & 0 & 2.354 & 4.565 & 1.664 & \mathrm{~N} \\ \text { HETATM } & 16 & \mathrm{~N} & 0 & 2.334 & 4.841 & -0.766 & \mathrm{~N} \\ \text { HETATM } & 17 & \mathrm{C} & 0 & 1.168 & 4.761 & -1.490 & \mathrm{C} \\ \text { HETATM } & 18 & \mathrm{~N} & 0 & 0.154 & 5.283 & -0.718 & \mathrm{~N} \\ \text { HETATM } & 19 & \mathrm{O} & 0 & 1.061 & 3.484 & 3.240 & \mathrm{O} \\ \text { HETATM } & 20 & \mathrm{O} & 0 & 1.067 & 4.379 & -2.636 & \mathrm{O} \\ \text { HETATM } & 21 & \mathrm{C} & 0 & 3.633 & 4.173 & 2.220 & \mathrm{C} \\ \text { HETATM } & 22 & \mathrm{C} & 0 & 3.618 & 4.540 & -1.371 & \mathrm{C} \\ \text { HETATM } & 23 & \mathrm{C} & 0 & -2.904 & 4.948 & 0.501 & \mathrm{C} \\ \text { HETATM } & 24 & \mathrm{C} & 0 & -4.187 & 4.029 & 0.399 & \mathrm{C} \\ \text { HETATM } & 25 & \mathrm{~N} & 0 & -4.005 & 3.108 & 1.498 & \\ \text { HETATM } & 26 & \mathrm{C} & 0 & -2.791 & 3.249 & 2.120 & \end{array}$




\begin{tabular}{|c|c|c|c|c|c|c|}
\hline HETATM & $27 \mathrm{~N}$ & 0 & -2.206 & 4.385 & 1.633 & $\mathrm{~N}$ \\
\hline HETATM & $28 \mathrm{~N}$ & 0 & -2.260 & 4.749 & -0.781 & $\mathrm{~N}$ \\
\hline HETATM & $29 \mathrm{C}$ & 0 & -2.922 & 3.854 & -1.586 & $\mathrm{C}$ \\
\hline HETATM & $30 \mathrm{~N}$ & 0 & -4.053 & 3.443 & -0.915 & $\mathrm{~N}$ \\
\hline HETATM & 310 & 0 & -2.352 & 2.529 & 2.997 & $\mathrm{O}$ \\
\hline HETATM & 320 & 0 & -2.615 & 3.539 & -2.717 & 4 \\
\hline HETATM & $33 \mathrm{C}$ & 0 & -5.863 & 0.788 & 0.084 & $\mathrm{C}$ \\
\hline HETATM & $34 \mathrm{C}$ & 0 & -5.863 & -0.785 & -0.084 & \\
\hline HETATM & $35 \mathrm{~N}$ & 0 & -5.147 & -1.239 & 1.094 & $\mathrm{~N}$ \\
\hline HETATM & $36 \mathrm{C}$ & 0 & -4.722 & -0.207 & 1.888 & $\mathrm{C}$ \\
\hline HETATM & $37 \mathrm{~N}$ & 0 & -5.171 & 0.963 & 1.338 & $\mathrm{~N}$ \\
\hline HETATM & $38 \mathrm{~N}$ & 0 & -5.147 & 1.241 & -1.094 & $\mathrm{~N}$ \\
\hline HETATM & $39 \mathrm{C}$ & 0 & -4.722 & 0.209 & -1.888 & $\mathrm{C}$ \\
\hline HETATM & $40 \mathrm{~N}$ & 0 & -1.261 & -1.305 & 4.515 & $\mathrm{~N}$ \\
\hline HETATM & $41 \mathrm{C}$ & 0 & -0.076 & -2.040 & 4.532 & $\mathrm{C}$ \\
\hline HETATM & $42 \mathrm{C}$ & 0 & 0.751 & -1.483 & 3.636 & $\mathrm{C}$ \\
\hline HETATM & $43 \mathrm{~N}$ & 0 & 0.053 & -0.416 & 3.067 & $\mathrm{~N}$ \\
\hline HETATM & $44 \mathrm{C}$ & 0 & -1.150 & -0.337 & 3.623 & $\mathrm{C}$ \\
\hline HETATM & $45 \mathrm{C}$ & 0 & 0.530 & 0.416 & 1.940 & $\mathrm{C}$ \\
\hline HETATM & $46 \mathrm{C}$ & 0 & 0.528 & -0.437 & 0.639 & $\mathrm{C}$ \\
\hline HETATM & $47 \mathrm{C}$ & 0 & 0.529 & 0.435 & -0.639 & $\mathrm{C}$ \\
\hline HETATM & $48 \mathrm{C}$ & 0 & 0.530 & -0.418 & -1.940 & $\mathrm{C}$ \\
\hline HETATM & $49 \mathrm{C}$ & 0 & -2.475 & -1.636 & 5.291 & $\mathrm{C}$ \\
\hline HETATM & $50 \mathrm{~N}$ & 0 & 0.053 & 0.416 & -3.067 & $\mathrm{~N}$ \\
\hline HETATM & $51 \mathrm{C}$ & 0 & -1.150 & 0.337 & -3.623 & $\mathrm{C}$ \\
\hline HETATM & $52 \mathrm{~N}$ & 0 & -1.260 & 1.306 & -4.514 & $\Lambda$ \\
\hline HETATM & $53 \mathrm{C}$ & 0 & -0.076 & 2.041 & -4.531 & $\mathrm{C}$ \\
\hline HETATM & $54 \mathrm{C}$ & 0 & 0.751 & 1.483 & -3.634 & $\mathrm{C}$ \\
\hline HETATM & $55 \mathrm{C}$ & 0 & -2.474 & 1.637 & -5.290 & C \\
\hline HETATM & $56 \mathrm{H}$ & 0 & -2.764 & 2.640 & -5.029 & $\mathrm{H}$ \\
\hline HETATM & $57 \mathrm{H}$ & 0 & -3.254 & 0.957 & -5.000 & $\mathrm{H}$ \\
\hline HETATM & $58 \mathrm{H}$ & 0 & -2.258 & 1.552 & -6.344 & $\mathrm{H}$ \\
\hline HETATM & $59 \mathrm{H}$ & 0 & -3.255 & -0.956 & 4.999 & $\mathrm{H}$ \\
\hline HETATM & $60 \mathrm{H}$ & 0 & -2.260 & -1.550 & 6.344 & $\mathrm{H}$ \\
\hline HETATM & $61 \mathrm{H}$ & 0 & -2.765 & -2.639 & 5.030 & $\mathrm{H}$ \\
\hline HETATM & $62 \mathrm{H}$ & 0 & 0.049 & 2.892 & -5.151 & $\mathrm{H}$ \\
\hline HETATM & $63 \mathrm{H}$ & 0 & 1.742 & 1.735 & -3.343 & $\mathrm{H}$ \\
\hline HETATM & $64 \mathrm{H}$ & 0 & -1.894 & -0.390 & -3.395 & \\
\hline HETATM & $65 \mathrm{H}$ & 0 & -0.146 & -1.254 & -1.873 & $\mathrm{H}$ \\
\hline HETATM & $66 \mathrm{H}$ & 0 & 1.509 & -0.783 & -2.197 & $\mathrm{H}$ \\
\hline HETATM & $67 \mathrm{H}$ & 0 & 1.382 & 1.099 & -0.650 & $\mathrm{H}$ \\
\hline HETATM & $68 \mathrm{H}$ & 0 & -0.362 & 1.049 & -0.641 & $\mathrm{H}$ \\
\hline HETATM & $69 \mathrm{H}$ & 0 & -0.364 & -1.049 & 0.642 & $\mathrm{H}$ \\
\hline HETATM & $70 \mathrm{H}$ & 0 & 1.380 & -1.102 & 0.650 & $\mathrm{H}$ \\
\hline HETATM & $71 \mathrm{H}$ & 0 & 1.510 & 0.781 & 2.196 & $\mathrm{H}$ \\
\hline HETATM & $72 \mathrm{H}$ & 0 & -0.145 & 1.253 & 1.873 & $\mathrm{H}$ \\
\hline
\end{tabular}




\begin{tabular}{|c|c|c|c|c|c|c|}
\hline HETATM & $73 \mathrm{H}$ & 0 & -1.894 & 0.390 & 3.394 & $\mathrm{H}$ \\
\hline HETATM & $74 \mathrm{H}$ & 0 & 1.742 & -1.736 & 3.344 & $\mathrm{H}$ \\
\hline HETATM & $75 \mathrm{H}$ & 0 & 0.049 & -2.891 & 5.153 & \\
\hline HETATM & $76 \mathrm{~N}$ & 0 & -5.171 & -0.961 & -1.338 & \\
\hline HETATM & $77 \mathrm{O}$ & 0 & -4.121 & -0.312 & 2.941 & \\
\hline HETATM & $78 \mathrm{O}$ & 0 & -4.120 & 0.314 & -2.941 & \\
\hline HETATM & $79 \mathrm{C}$ & 0 & -5.022 & 2.226 & 2.029 & \\
\hline HETATM & $80 \mathrm{C}$ & 0 & -5.062 & 2.623 & -1.552 & \\
\hline HETATM & $81 \mathrm{C}$ & 0 & -1.119 & 5.039 & 2.337 & \\
\hline НETATM & $82 \mathrm{C}$ & 0 & -1.154 & 5.552 & -1.287 & \\
\hline HETATM & $83 \mathrm{C}$ & 0 & -4.189 & -4.027 & -0.399 & \\
\hline HETATM & $84 \mathrm{C}$ & 0 & -2.907 & -4.947 & -0.501 & \\
\hline HETATM & $85 \mathrm{~N}$ & 0 & -2.262 & -4.748 & 0.781 & \\
\hline HETATM & $86 \mathrm{C}$ & 0 & -2.924 & -3.852 & 1.586 & \\
\hline HETATM & $87 \mathrm{~N}$ & 0 & -4.054 & -3.441 & 0.915 & \\
\hline HETATM & $88 \mathrm{~N}$ & 0 & -4.007 & -3.106 & -1.498 & \\
\hline HETATM & $89 \mathrm{C}$ & 0 & -2.792 & -3.248 & -2.120 & \\
\hline HETATM & $90 \mathrm{~N}$ & 0 & -2.208 & -4.385 & -1.633 & \\
\hline HETATM & 910 & 0 & -2.617 & -3.538 & 2.717 & \\
\hline HETATM & $92 \mathrm{O}$ & 0 & -2.353 & -2.528 & -2.997 & \\
\hline HETATM & $93 \mathrm{C}$ & 0 & 0.606 & -5.743 & -0.579 & \\
\hline HETATM & $94 \mathrm{C}$ & 0 & 2.161 & -5.448 & -0.536 & \\
\hline HETATM & $95 \mathrm{~N}$ & 0 & 2.332 & -4.842 & 0.766 & \\
\hline HETATM & $96 \mathrm{C}$ & 0 & 1.166 & -4.761 & 1.490 & \\
\hline HETATM & $97 \mathrm{~N}$ & 0 & 0.152 & -5.283 & 0.718 & \\
\hline HETATM & $98 \mathrm{~N}$ & 0 & 0.172 & -4.955 & -1.707 & \\
\hline HETATM & $99 \mathrm{C}$ & 0 & 1.181 & -4.227 & -2.292 & \\
\hline HETATM & $100 \mathrm{~N}$ & 0 & 2.352 & -4.566 & -1.664 & \\
\hline HETATM & $101 \mathrm{O}$ & 0 & 1.065 & -4.379 & 2.636 & \\
\hline HETATM & $102 \mathrm{O}$ & 0 & 1.059 & -3.485 & -3.240 & \\
\hline HETATM & $103 \mathrm{C}$ & 0 & -1.157 & -5.551 & 1.287 & \\
\hline HETATM & $104 \mathrm{C}$ & 0 & -1.122 & -5.039 & -2.337 & \\
\hline HETATM & $105 \mathrm{C}$ & 0 & -5.063 & -2.621 & 1.551 & \\
\hline HETATM & $106 \mathrm{C}$ & 0 & -5.023 & -2.223 & -2.029 & \\
\hline HETATM & $107 \mathrm{C}$ & 0 & 3.631 & -4.175 & -2.220 & \\
\hline HETATM & $108 \mathrm{C}$ & 0 & 3.616 & -4.541 & 1.371 & \\
\hline HETATM & $109 \mathrm{C}$ & 0 & 5.728 & 0.176 & -1.808 & \\
\hline HETATM & $110 \mathrm{C}$ & 0 & 5.728 & -0.179 & 1.807 & \\
\hline HETATM & $111 \mathrm{C}$ & 0 & 5.754 & -1.816 & -0.168 & \\
\hline HETATM & $112 \mathrm{C}$ & 0 & 5.072 & -3.240 & -0.313 & \\
\hline HETATM & $113 \mathrm{~N}$ & 0 & 4.278 & -3.337 & 0.896 & \\
\hline HETATM & $114 \mathrm{~N}$ & 0 & 5.253 & -1.356 & 1.108 & \\
\hline HETATM & $115 \mathrm{~N}$ & 0 & 5.242 & -1.099 & -1.315 & \\
\hline HETATM & $116 \mathrm{C}$ & 0 & 4.336 & -1.828 & -2.045 & \\
\hline HETATM & $117 \mathrm{~N}$ & 0 & 4.311 & -3.099 & -1.530 & \\
\hline HETATM & $118 \mathrm{C}$ & 0 & 4.344 & -2.211 & 1.675 & \\
\hline
\end{tabular}




\begin{tabular}{|c|c|c|c|c|c|}
\hline HETATM 119 O & 0 & 3.718 & -1.436 & -3.013 & \\
\hline HETATM 120 O & 0 & 3.755 & -2.034 & 2.724 & \\
\hline HETATM $121 \mathrm{H}$ & 0 & 6.833 & 1.840 & 0.174 & \\
\hline HETATM $122 \mathrm{H}$ & 0 & 5.774 & 4.054 & 0.381 & \\
\hline HETATM $123 \mathrm{H}$ & 0 & 2.775 & 6.330 & 0.632 & \\
\hline HETATM $124 \mathrm{H}$ & 0 & 0.369 & 6.785 & 0.721 & \\
\hline HETATM $125 \mathrm{H}$ & 0 & 4.283 & 5.037 & 2.236 & \\
\hline HETATM $126 \mathrm{H}$ & 0 & 3.442 & 3.827 & 3.222 & \\
\hline HETATM $127 \mathrm{H}$ & 0 & 3.437 & 4.405 & -2.424 & \\
\hline HETATM $128 \mathrm{H}$ & 0 & 4.283 & 5.377 & -1.211 & \\
\hline HETATM $129 \mathrm{H}$ & 0 & -3.125 & 5.991 & 0.664 & \\
\hline HETATM $130 \mathrm{H}$ & 0 & -5.120 & 4.563 & 0.493 & \\
\hline HETATM $131 \mathrm{H}$ & 0 & -6.848 & 1.224 & 0.123 & \\
\hline HETATM $132 \mathrm{H}$ & 0 & -6.849 & -1.221 & -0.123 & \\
\hline HETATM $133 \mathrm{H}$ & 0 & -5.970 & 2.746 & 2.009 & \\
\hline HETATM $134 \mathrm{H}$ & 0 & -4.749 & 1.992 & 3.043 & \\
\hline HETATM $135 \mathrm{H}$ & 0 & -4.818 & 2.589 & -2.599 & \\
\hline HETATM $136 \mathrm{H}$ & 0 & -6.029 & 3.086 & -1.408 & \\
\hline HETATM $137 \mathrm{H}$ & 0 & -1.027 & 4.555 & 3.295 & \\
\hline HETATM $138 \mathrm{H}$ & 0 & -1.382 & 6.081 & 2.466 & \\
\hline HETATM $139 \mathrm{H}$ & 0 & -1.080 & 5.345 & -2.342 & \\
\hline HETATM $140 \mathrm{H}$ & 0 & -1.393 & 6.596 & -1.126 & \\
\hline HETATM $141 \mathrm{H}$ & 0 & -5.122 & -4.561 & -0.494 & \\
\hline HETATM $142 \mathrm{H}$ & 0 & -3.128 & -5.990 & -0.663 & \\
\hline HETATM $143 \mathrm{H}$ & 0 & 0.366 & -6.785 & -0.720 & \\
\hline HETATM $144 \mathrm{H}$ & 0 & 2.773 & -6.331 & -0.632 & \\
\hline HETATM $145 \mathrm{H}$ & 0 & -1.395 & -6.595 & 1.126 & \\
\hline HETATM $146 \mathrm{H}$ & 0 & -1.083 & -5.344 & 2.342 & \\
\hline HETATM $147 \mathrm{H}$ & 0 & -1.029 & -4.556 & -3.296 & \\
\hline HETATM $148 \mathrm{H}$ & 0 & -1.385 & -6.081 & -2.465 & \\
\hline HETATM $149 \mathrm{H}$ & 0 & -6.030 & -3.083 & 1.408 & \\
\hline HETATM $150 \mathrm{H}$ & 0 & -4.819 & -2.587 & 2.599 & \\
\hline HETATM $151 \mathrm{H}$ & 0 & -4.749 & -1.990 & -3.043 & \\
\hline HETATM $152 \mathrm{H}$ & 0 & -5.971 & -2.743 & -2.009 & \\
\hline HETATM $153 \mathrm{H}$ & 0 & 3.440 & -3.828 & -3.222 & \\
\hline HETATM $154 \mathrm{H}$ & 0 & 4.281 & -5.039 & -2.236 & \\
\hline HETATM $155 \mathrm{H}$ & 0 & 3.435 & -4.406 & 2.424 & \\
\hline HETATM $156 \mathrm{H}$ & 0 & 4.281 & -5.379 & 1.211 & \\
\hline HETATM $157 \mathrm{H}$ & 0 & 5.388 & 0.264 & -2.826 & \\
\hline HETATM $158 \mathrm{H}$ & 0 & 6.809 & 0.169 & -1.770 & \\
\hline HETATM $159 \mathrm{H}$ & 0 & 5.389 & -0.266 & 2.826 & \\
\hline HETATM $160 \mathrm{H}$ & 0 & 6.809 & -0.172 & 1.770 & \\
\hline HETATM $161 \mathrm{H}$ & 0 & 6.832 & -1.843 & -0.174 & \\
\hline HETATM $162 \mathrm{H}$ & 0 & 5.772 & -4.057 & -0.381 & \\
\hline
\end{tabular}




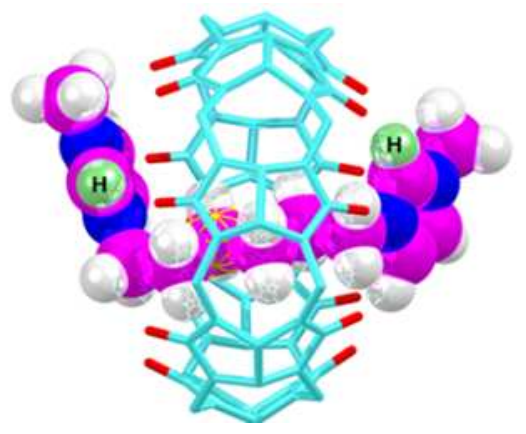

DIC6-CB7

SCF Done: $E=-4922.26672421$

REMARK 1 File created by GaussView 5.0.8

$\begin{array}{llllrrrr}\text { HETATM } & 1 & \mathrm{C} & 0 & 4.140 & -4.257 & 0.354 & \mathrm{C} \\ \text { HETATM } & 2 & \mathrm{C} & 0 & 2.794 & -5.085 & 0.399 & \mathrm{C} \\ \text { HETATM } & 3 & \mathrm{~N} & 0 & 2.150 & -4.714 & -0.841 & \mathrm{~N} \\ \text { HETATM } & 4 & \mathrm{C} & 0 & 2.847 & -3.768 & -1.555 & \mathrm{C} \\ \text { HETATM } & 5 & \mathrm{~N} & 0 & 4.009 & -3.496 & -0.876 & \mathrm{~N} \\ \text { HETATM } & 6 & \mathrm{~N} & 0 & 4.085 & -3.495 & 1.576 & \mathrm{~N} \\ \text { HETATM } & 7 & \mathrm{C} & 0 & 2.905 & -3.642 & 2.258 & \mathrm{C} \\ \text { HETATM } & 8 & \mathrm{~N} & 0 & 2.161 & -4.592 & 1.600 & \mathrm{~N} \\ \text { HETATM } & 9 & \mathrm{O} & 0 & 2.519 & -3.317 & -2.637 & \mathrm{O} \\ \text { HETATM } & 10 & \mathrm{O} & 0 & 2.597 & -3.101 & 3.298 & \mathrm{O} \\ \text { HETATM } & 11 & \mathrm{C} & 0 & -0.761 & -5.602 & 0.449 & \mathrm{C} \\ \text { HETATM } & 12 & \mathrm{C} & 0 & -2.293 & -5.219 & 0.425 & \mathrm{C} \\ \text { HETATM } & 13 & \mathrm{~N} & 0 & -2.454 & -4.607 & -0.875 & \mathrm{~N} \\ \text { HETATM } & 14 & \mathrm{C} & 0 & -1.273 & -4.504 & -1.571 & \mathrm{C} \\ \text { HETATM } & 15 & \mathrm{~N} & 0 & -0.288 & -5.100 & -0.824 & \mathrm{~N} \\ \text { HETATM } & 16 & \mathrm{~N} & 0 & -0.274 & -4.896 & 1.610 & \mathrm{~N} \\ \text { HETATM } & 17 & \mathrm{C} & 0 & -1.212 & -4.073 & 2.178 & \mathrm{C} \\ \text { HETATM } & 18 & \mathrm{~N} & 0 & -2.409 & -4.319 & 1.550 & \mathrm{~N} \\ \text { HETATM } & 19 & \mathrm{O} & 0 & -1.139 & -4.049 & -2.689 & \mathrm{O} \\ \text { HETATM } & 20 & \mathrm{O} & 0 & -1.042 & -3.323 & 3.121 & \mathrm{O} \\ \text { HETATM } & 21 & \mathrm{C} & 0 & 1.009 & -5.405 & -1.410 & \mathrm{C} \\ \text { HETATM } & 22 & \mathrm{C} & 0 & 1.001 & -5.167 & 2.245 & \mathrm{C} \\ \text { HETATM } & 23 & \mathrm{C} & 0 & -5.099 & -2.912 & 0.206 & \mathrm{C} \\ \text { HETATM } & 24 & \mathrm{C} & 0 & -5.749 & -1.478 & 0.062 & \mathrm{C} \\ \text { HETATM } & 25 & \mathrm{~N} & 0 & -5.272 & -1.041 & -1.232 & \mathrm{~N} \\ \text { HETATM } & 26 & \mathrm{C} & 0 & -4.491 & -1.974 & -1.867 & \mathrm{C} \\ \text { HETATM } & 27 & \mathrm{~N} & 0 & -4.364 & -3.058 & -1.032 & \mathrm{~N} \\ \text { HETATM } & 28 & \mathrm{~N} & 0 & -4.306 & -2.777 & 1.407 & \mathrm{~N} \\ \text { HETATM } & 29 & \mathrm{C} & 0 & -4.363 & -1.527 & 1.961 & \mathrm{C} \\ \text { HETATM } & 30 & \mathrm{~N} & 0 & -5.225 & -0.772 & 1.212 & \mathrm{~N} \\ \text { HETATM } & 31 & \mathrm{O} & 0 & -4.069 & -1.897 & -3.004 & \mathrm{O} \\ \text { HETATM } & 32 & \mathrm{O} & 0 & -3.816 & -1.185 & 2.993 & \mathrm{O} \\ \text { HETATM } & 33 & \mathrm{C} & 0 & -5.502 & 2.167 & -0.288 & \mathrm{C}\end{array}$




\begin{tabular}{|c|c|c|c|c|c|c|}
\hline HETATM & $34 \mathrm{C}$ & 0 & -4.660 & 3.497 & -0.419 & $\mathrm{C}$ \\
\hline HETATM & $35 \mathrm{~N}$ & 0 & -3.843 & 3.238 & -1.586 & $\mathrm{~N}$ \\
\hline HETATM & $36 \mathrm{C}$ & 0 & -4.106 & 2.028 & -2.177 & $\mathrm{C}$ \\
\hline HETATM & $37 \mathrm{~N}$ & 0 & -5.077 & 1.398 & -1.438 & \\
\hline HETATM & $38 \mathrm{~N}$ & 0 & -5.093 & 1.666 & 1.005 & 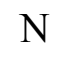 \\
\hline HETATM & $39 \mathrm{C}$ & 0 & -4.218 & 2.496 & 1.665 & $\mathrm{C}$ \\
\hline HETATM & $40 \mathrm{~N}$ & 0 & -1.252 & -1.608 & -4.751 & $\mathrm{~N}$ \\
\hline HETATM & $41 \mathrm{C}$ & 0 & -1.937 & -0.412 & -4.563 & \\
\hline HETATM & $42 \mathrm{C}$ & 0 & -1.038 & 0.493 & -4.146 & \\
\hline HETATM & $43 \mathrm{~N}$ & 0 & 0.187 & -0.163 & -4.071 & $1 \mathrm{~V}$ \\
\hline HETATM & $44 \mathrm{C}$ & 0 & 0.020 & -1.430 & -4.422 & $C$ \\
\hline HETATM & $45 \mathrm{C}$ & 0 & 1.444 & 0.481 & -3.605 & $\mathrm{C}$ \\
\hline HETATM & $46 \mathrm{C}$ & 0 & 1.843 & -0.034 & -2.218 & \\
\hline HETATM & $47 \mathrm{C}$ & 0 & 0.786 & 0.202 & -1.129 & $\mathrm{C}$ \\
\hline HETATM & $48 \mathrm{C}$ & 0 & 1.323 & -0.237 & 0.254 & $C$ \\
\hline HETATM & $49 \mathrm{C}$ & 0 & -1.877 & -2.852 & -5.248 & \\
\hline HETATM & $50 \mathrm{C}$ & 0 & 0.333 & 0.128 & 1.371 & $\mathrm{C}$ \\
\hline HETATM & $51 \mathrm{C}$ & 0 & 0.818 & -0.260 & 2.783 & $\mathrm{C}$ \\
\hline HETATM & $52 \mathrm{~N}$ & 0 & -0.132 & 0.352 & 3.744 & $\mathrm{~N}$ \\
\hline HETATM & $53 \mathrm{C}$ & 0 & -1.247 & -0.203 & 4.191 & $\mathrm{C}$ \\
\hline HETATM & $54 \mathrm{~N}$ & 0 & -1.921 & 0.709 & 4.874 & $\mathrm{~N}$ \\
\hline HETATM & $55 \mathrm{C}$ & 0 & -1.212 & 1.910 & 4.843 & $\mathrm{C}$ \\
\hline HETATM & $56 \mathrm{C}$ & 0 & -0.092 & 1.689 & 4.139 & $\mathrm{C}$ \\
\hline HETATM & $57 \mathrm{C}$ & 0 & -3.261 & 0.546 & 5.474 & $\Omega$ \\
\hline HETATM & $58 \mathrm{H}$ & 0 & -3.194 & 0.675 & 6.542 & $\mathrm{H}$ \\
\hline HETATM & $59 \mathrm{H}$ & 0 & -3.627 & -0.434 & 5.227 & $\mathrm{H}$ \\
\hline HETATM & $60 \mathrm{H}$ & 0 & -3.904 & 1.286 & 5.031 & $\mathrm{H}$ \\
\hline HETATM & $61 \mathrm{H}$ & 0 & -1.265 & -3.687 & -4.966 & $\mathrm{H}$ \\
\hline HETATM & $62 \mathrm{H}$ & 0 & -1.984 & -2.788 & -6.320 & \\
\hline HETATM & $63 \mathrm{H}$ & 0 & -2.836 & -2.935 & -4.771 & $\mathrm{H}$ \\
\hline HETATM & $64 \mathrm{H}$ & 0 & -1.589 & 2.795 & 5.288 & $\mathrm{H}$ \\
\hline HETATM & $65 \mathrm{H}$ & 0 & 0.699 & 2.332 & 3.838 & $\mathrm{H}$ \\
\hline HETATM & $66 \mathrm{H}$ & 0 & -1.553 & -1.202 & 3.989 & $\mathrm{H}$ \\
\hline HETATM & $67 \mathrm{H}$ & 0 & 1.800 & 0.127 & 2.992 & $\mathrm{H}$ \\
\hline HETATM & $68 \mathrm{H}$ & 0 & 0.824 & -1.325 & 2.944 & $\mathrm{H}$ \\
\hline HETATM & $69 \mathrm{H}$ & 0 & -0.624 & -0.347 & 1.189 & $\mathrm{H}$ \\
\hline HETATM & $70 \mathrm{H}$ & 0 & 0.174 & 1.198 & 1.357 & $\mathrm{H}$ \\
\hline HETATM & $71 \mathrm{H}$ & 0 & 2.268 & 0.257 & 0.440 & $\mathrm{H}$ \\
\hline HETATM & $72 \mathrm{H}$ & 0 & 1.509 & -1.305 & 0.247 & $\mathrm{H}$ \\
\hline HETATM & $73 \mathrm{H}$ & 0 & -0.121 & -0.345 & -1.352 & . \\
\hline HETATM & $74 \mathrm{H}$ & 0 & 0.529 & 1.254 & -1.098 & $\mathrm{H}$ \\
\hline HETATM & $75 \mathrm{H}$ & 0 & 2.737 & 0.502 & -1.965 & $\mathrm{H}$ \\
\hline HETATM & $76 \mathrm{H}$ & 0 & 2.089 & -1.081 & -2.285 & $\mathrm{H}$ \\
\hline HETATM & $77 \mathrm{H}$ & 0 & 1.258 & 1.537 & -3.588 & $\mathrm{H}$ \\
\hline HETATM & $78 \mathrm{H}$ & 0 & 2.230 & 0.272 & -4.312 & $\mathrm{H}$ \\
\hline HETATM & $79 \mathrm{H}$ & 0 & 0.773 & -2.182 & -4.391 & 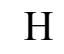 \\
\hline
\end{tabular}




\begin{tabular}{|c|c|c|c|c|c|c|}
\hline HETATM & $80 \mathrm{H}$ & 0 & -1.134 & 1.513 & -3.872 & $\mathrm{H}$ \\
\hline HETATM & $81 \mathrm{H}$ & 0 & -2.986 & -0.339 & -4.673 & $\mathrm{H}$ \\
\hline HETATM & $82 \mathrm{~N}$ & 0 & -3.967 & 3.571 & 0.847 & $\mathrm{~N}$ \\
\hline HETATM & $83 \mathrm{O}$ & 0 & -3.635 & 1.630 & -3.223 & \\
\hline HETATM & $84 \mathrm{O}$ & 0 & -3.819 & 2.351 & 2.801 & \\
\hline HETATM & $85 \mathrm{C}$ & 0 & -5.703 & 0.174 & -1.901 & \\
\hline HETATM & $86 \mathrm{C}$ & 0 & -5.688 & 0.527 & 1.668 & $C$ \\
\hline HETATM & $87 \mathrm{C}$ & 0 & -3.735 & -4.289 & -1.479 & \\
\hline HETATM & $88 \mathrm{C}$ & 0 & -3.666 & -3.871 & 2.106 & \\
\hline HETATM & $89 \mathrm{C}$ & 0 & -1.632 & 5.478 & -0.510 & C \\
\hline HETATM & $90 \mathrm{C}$ & 0 & -0.071 & 5.721 & -0.452 & O \\
\hline HETATM & $91 \mathrm{~N}$ & 0 & 0.424 & 4.905 & -1.540 & \\
\hline HETATM & $92 \mathrm{C}$ & 0 & -0.563 & 4.179 & -2.157 & \\
\hline HETATM & $93 \mathrm{~N}$ & 0 & -1.766 & 4.551 & -1.613 & \\
\hline HETATM & $94 \mathrm{~N}$ & 0 & -1.924 & 4.942 & 0.799 & \\
\hline HETATM & $95 \mathrm{C}$ & 0 & -0.808 & 4.802 & 1.591 & $\mathrm{C}$ \\
\hline HETATM & $96 \mathrm{~N}$ & 0 & 0.270 & 5.264 & 0.875 & \\
\hline HETATM & $97 \mathrm{O}$ & 0 & -0.405 & 3.407 & -3.085 & \\
\hline HETATM & $98 \mathrm{O}$ & 0 & -0.789 & 4.424 & 2.742 & 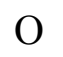 \\
\hline HETATM & $99 \mathrm{C}$ & 0 & 3.412 & 4.785 & -0.165 & \\
\hline HETATM & $100 \mathrm{C}$ & 0 & 4.617 & 3.779 & 0.015 & \\
\hline HETATM & $101 \mathrm{~N}$ & 0 & 4.595 & 3.017 & -1.214 & \\
\hline HETATM & $102 \mathrm{C}$ & 0 & 3.511 & 3.310 & -2.002 & \\
\hline HETATM & $103 \mathrm{~N}$ & 0 & 2.817 & 4.335 & -1.406 & \\
\hline HETATM & $104 \mathrm{~N}$ & 0 & 2.617 & 4.554 & 1.019 & \\
\hline HETATM & $105 \mathrm{C}$ & 0 & 3.049 & 3.475 & 1.751 & \\
\hline HETATM & $106 \mathrm{~N}$ & 0 & 4.234 & 3.050 & 1.204 & \\
\hline HETATM & $107 \mathrm{O}$ & 0 & 3.249 & 2.806 & -3.075 & \\
\hline HETATM & $108 \mathrm{O}$ & 0 & 2.523 & 3.032 & 2.752 & \\
\hline HETATM & $109 \mathrm{C}$ & 0 & 1.755 & 5.020 & -2.113 & \\
\hline HETATM & $110 \mathrm{C}$ & 0 & 1.566 & 5.431 & 1.499 & \\
\hline HETATM & $111 \mathrm{C}$ & 0 & -3.026 & 4.230 & -2.256 & \\
\hline HETATM & $112 \mathrm{C}$ & 0 & -3.255 & 4.739 & 1.338 & \\
\hline HETATM & $113 \mathrm{C}$ & 0 & 5.114 & 2.149 & 1.919 & \\
\hline HETATM & $114 \mathrm{C}$ & 0 & 5.637 & 2.105 & -1.668 & \\
\hline HETATM & $115 \mathrm{C}$ & 0 & 5.161 & -2.688 & 2.112 & \\
\hline HETATM & $116 \mathrm{C}$ & 0 & 5.102 & -2.754 & -1.493 & \\
\hline HETATM & $117 \mathrm{C}$ & 0 & 6.045 & -1.122 & 0.276 & \\
\hline HETATM & $118 \mathrm{C}$ & 0 & 6.110 & 0.459 & 0.251 & \\
\hline HETATM & $119 \mathrm{~N}$ & 0 & 5.625 & 0.782 & -1.070 & \\
\hline HETATM & $120 \mathrm{~N}$ & 0 & 5.352 & -1.424 & -0.965 & \\
\hline HETATM & $121 \mathrm{~N}$ & 0 & 5.320 & -1.391 & 1.491 & \\
\hline HETATM & $122 \mathrm{C}$ & 0 & 4.792 & -0.264 & 2.066 & \\
\hline HETATM & $123 \mathrm{~N}$ & 0 & 5.242 & 0.821 & 1.354 & \\
\hline HETATM & $124 \mathrm{C}$ & 0 & 5.194 & -0.320 & -1.771 & \\
\hline HETATM & $125 \mathrm{O}$ & 0 & 4.118 & -0.221 & 3.073 & \\
\hline
\end{tabular}




\begin{tabular}{|c|c|c|c|c|}
\hline HETATM 126 O & 0 & 4.804 & -0.327 & -2.919 \\
\hline HETATM $127 \mathrm{H}$ & 0 & 5.027 & -4.871 & 0.324 \\
\hline HETATM $128 \mathrm{H}$ & 0 & 2.942 & -6.153 & 0.448 \\
\hline HETATM $129 \mathrm{H}$ & 0 & -0.576 & -6.661 & 0.539 \\
\hline HETATM $130 \mathrm{H}$ & 0 & -2.957 & -6.062 & 0.537 \\
\hline HETATM $131 \mathrm{H}$ & 0 & 1.179 & -6.470 & -1.325 \\
\hline HETATM $132 \mathrm{H}$ & 0 & 0.952 & -5.119 & -2.446 \\
\hline HETATM $133 \mathrm{H}$ & 0 & 0.963 & -4.744 & 3.235 \\
\hline HETATM $134 \mathrm{H}$ & 0 & 1.125 & -6.241 & 2.301 \\
\hline HETATM $135 \mathrm{H}$ & 0 & -5.822 & -3.706 & 0.315 \\
\hline HETATM $136 \mathrm{H}$ & 0 & -6.827 & -1.487 & 0.085 \\
\hline HETATM $137 \mathrm{H}$ & 0 & -6.569 & 2.326 & -0.315 \\
\hline HETATM $138 \mathrm{H}$ & 0 & -5.266 & 4.377 & -0.571 \\
\hline HETATM $139 \mathrm{H}$ & 0 & -6.774 & 0.271 & -1.787 \\
\hline HETATM $140 \mathrm{H}$ & 0 & -5.451 & 0.062 & -2.941 \\
\hline HETATM $141 \mathrm{H}$ & 0 & -5.433 & 0.605 & 2.710 \\
\hline HETATM $142 \mathrm{H}$ & 0 & -6.762 & 0.567 & 1.544 \\
\hline HETATM $143 \mathrm{H}$ & 0 & -3.569 & -4.187 & -2.537 \\
\hline HETATM $144 \mathrm{H}$ & 0 & -4.412 & -5.111 & -1.288 \\
\hline HETATM $145 \mathrm{H}$ & 0 & -3.467 & -3.528 & 3.107 \\
\hline HETATM $146 \mathrm{H}$ & 0 & -4.348 & -4.711 & 2.131 \\
\hline HETATM $147 \mathrm{H}$ & 0 & -2.205 & 6.374 & -0.696 \\
\hline HETATM $148 \mathrm{H}$ & 0 & 0.214 & 6.752 & -0.591 \\
\hline HETATM $149 \mathrm{H}$ & 0 & 3.711 & 5.819 & -0.231 \\
\hline HETATM $150 \mathrm{H}$ & 0 & 5.573 & 4.261 & 0.151 \\
\hline HETATM $151 \mathrm{H}$ & 0 & 1.999 & 6.073 & -2.175 \\
\hline HETATM $152 \mathrm{H}$ & 0 & 1.718 & 4.591 & -3.100 \\
\hline HETATM $153 \mathrm{H}$ & 0 & 1.428 & 5.225 & 2.547 \\
\hline HETATM $154 \mathrm{H}$ & 0 & 1.893 & 6.452 & 1.361 \\
\hline HETATM $155 \mathrm{H}$ & 0 & -3.598 & 5.144 & -2.348 \\
\hline HETATM $156 \mathrm{H}$ & 0 & -2.802 & 3.838 & -3.233 \\
\hline HETATM $157 \mathrm{H}$ & 0 & -3.140 & 4.608 & 2.401 \\
\hline HETATM $158 \mathrm{H}$ & 0 & -3.852 & 5.617 & 1.130 \\
\hline HETATM $159 \mathrm{H}$ & 0 & 4.705 & 2.025 & 2.907 \\
\hline HETATM $160 \mathrm{H}$ & 0 & 6.096 & 2.602 & 1.973 \\
\hline HETATM $161 \mathrm{H}$ & 0 & 5.489 & 1.965 & -2.725 \\
\hline HETATM $162 \mathrm{H}$ & 0 & 6.597 & 2.564 & -1.478 \\
\hline HETATM $163 \mathrm{H}$ & 0 & 4.930 & -2.516 & 3.149 \\
\hline HETATM $164 \mathrm{H}$ & 0 & 6.090 & -3.233 & 2.017 \\
\hline HETATM $165 \mathrm{H}$ & 0 & 4.851 & -2.624 & -2.533 \\
\hline HETATM $166 \mathrm{H}$ & 0 & 6.003 & -3.347 & -1.399 \\
\hline HETATM $167 \mathrm{H}$ & 0 & 7.015 & -1.594 & 0.297 \\
\hline $\begin{array}{l}\text { HETATM } 168 \mathrm{H} \\
\text { FND }\end{array}$ & 0 & 7.103 & 0.852 & 0.401 \\
\hline
\end{tabular}




\section{DIC8-CB7}

SCF Done: $E=-4999.94969244$

REMARK 1 File created by GaussView 5.0.8

\begin{tabular}{|c|c|c|c|c|c|c|}
\hline HETATM & $1 \mathrm{C}$ & 0 & 2.636 & -5.143 & 0.158 & $\mathrm{C}$ \\
\hline HETATM & $2 \mathrm{C}$ & 0 & 1.109 & -5.524 & -0.006 & $\mathrm{C}$ \\
\hline HETATM & $3 \mathrm{~N}$ & 0 & 0.761 & -4.899 & -1.263 & $\mathrm{~N}$ \\
\hline HETATM & $4 \mathrm{C}$ & 0 & 1.819 & -4.244 & -1.858 & $\mathrm{C}$ \\
\hline HETATM & $5 \mathrm{~N}$ & 0 & 2.919 & -4.404 & -1.052 & $\mathrm{~N}$ \\
\hline HETATM & $6 \mathrm{~N}$ & 0 & 2.639 & -4.376 & 1.383 & $\mathrm{~N}$ \\
\hline HETATM & $7 \mathrm{C}$ & 0 & 1.396 & -4.219 & 1.932 & $\mathrm{C}$ \\
\hline HETATM & $8 \mathrm{~N}$ & 0 & 0.508 & -4.939 & 1.169 & $\mathrm{~N}$ \\
\hline HETATM & 90 & 0 & 1.790 & -3.710 & -2.947 & $\mathrm{O}$ \\
\hline HETATM & $10 \mathrm{O}$ & 0 & 1.126 & -3.629 & 2.959 & $\mathrm{O}$ \\
\hline HETATM & $11 \mathrm{C}$ & 0 & -2.406 & -5.027 & -0.334 & $\mathrm{C}$ \\
\hline HETATM & $12 \mathrm{C}$ & 0 & -3.785 & -4.269 & -0.451 & $\mathrm{C}$ \\
\hline HETATM & $13 \mathrm{~N}$ & 0 & -3.596 & -3.439 & -1.621 & $\mathrm{~N}$ \\
\hline HETATM & $14 \mathrm{C}$ & 0 & -2.367 & -3.616 & -2.218 & $\mathrm{C}$ \\
\hline HETATM & $15 \mathrm{~N}$ & 0 & -1.673 & -4.547 & -1.485 & $\mathrm{~N}$ \\
\hline HETATM & $16 \mathrm{~N}$ & 0 & -1.915 & -4.594 & 0.958 & $\mathrm{~N}$ \\
\hline HETATM & $17 \mathrm{C}$ & 0 & -2.749 & -3.710 & 1.588 & $\mathrm{C}$ \\
\hline HETATM & $18 \mathrm{~N}$ & 0 & -3.871 & -3.570 & 0.809 & $\mathrm{~N}$ \\
\hline HETATM & $19 \mathrm{O}$ & 0 & -2.004 & -3.114 & -3.262 & $\mathrm{O}$ \\
\hline HETATM & $20 \mathrm{O}$ & 0 & -2.576 & -3.210 & 2.685 & $\mathrm{O}$ \\
\hline HETATM & $21 \mathrm{C}$ & 0 & -0.458 & -5.159 & -2.003 & $\mathrm{C}$ \\
\hline HETATM & $22 \mathrm{C}$ & 0 & -0.817 & -5.231 & 1.663 & $\mathrm{C}$ \\
\hline HETATM & $23 \mathrm{C}$ & 0 & -5.823 & -1.215 & -0.502 & $\mathrm{C}$ \\
\hline HETATM & $24 \mathrm{C}$ & 0 & -5.961 & 0.357 & -0.422 & $\mathrm{C}$ \\
\hline HETATM & $25 \mathrm{~N}$ & 0 & -5.280 & 0.802 & -1.615 & $\mathrm{~N}$ \\
\hline HETATM & $26 \mathrm{C}$ & 0 & -4.721 & -0.225 & -2.331 & $\mathrm{C}$ \\
\hline HETATM & $27 \mathrm{~N}$ & 0 & -4.976 & -1.399 & -1.665 & $\mathrm{~N}$ \\
\hline HETATM & $28 \mathrm{~N}$ & 0 & -5.242 & -1.547 & 0.777 & $\mathrm{~N}$ \\
\hline HETATM & $29 \mathrm{C}$ & 0 & -4.922 & -0.449 & 1.528 & $\mathrm{C}$ \\
\hline HETATM & $30 \mathrm{~N}$ & 0 & -5.323 & 0.663 & 0.844 & $\mathrm{~N}$ \\
\hline HETATM & 310 & 0 & -4.183 & -0.124 & -3.416 & $\mathrm{O}$ \\
\hline HETATM & $32 \mathrm{O}$ & 0 & -4.452 & -0.474 & 2.653 & $\mathrm{O}$ \\
\hline HETATM & $33 \mathrm{C}$ & 0 & -4.498 & 3.719 & -0.236 & $\mathrm{C}$ \\
\hline HETATM & $34 \mathrm{C}$ & 0 & -3.257 & 4.696 & -0.166 & $\mathrm{C}$ \\
\hline
\end{tabular}




\begin{tabular}{|c|c|c|c|c|c|c|}
\hline HETATM & $35 \mathrm{~N}$ & 0 & -2.536 & 4.381 & -1.383 & $N$ \\
\hline HETATM & $36 \mathrm{C}$ & 0 & -3.160 & 3.434 & -2.154 & $\mathrm{C}$ \\
\hline HETATM & $37 \mathrm{~N}$ & 0 & -4.312 & 3.056 & -1.508 & 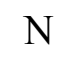 \\
\hline HETATM & $38 \mathrm{~N}$ & 0 & -4.319 & 2.897 & 0.937 & $\mathrm{~N}$ \\
\hline HETATM & $39 \mathrm{C}$ & 0 & -3.207 & 3.236 & 1.680 & $\mathrm{C}$ \\
\hline HETATM & $40 \mathrm{~N}$ & 0 & -1.539 & -0.395 & -5.383 & \\
\hline HETATM & $41 \mathrm{C}$ & 0 & -1.766 & 0.883 & -4.881 & \\
\hline HETATM & $42 \mathrm{C}$ & 0 & -0.567 & 1.390 & -4.543 & $\mathrm{C}$ \\
\hline HETATM & $43 \mathrm{~N}$ & 0 & 0.379 & 0.411 & -4.835 & $\mathrm{~N}$ \\
\hline HETATM & $44 \mathrm{C}$ & 0 & -0.239 & -0.647 & -5.327 & 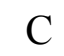 \\
\hline HETATM & $45 \mathrm{C}$ & 0 & 1.822 & 0.470 & -4.472 & $\mathrm{C}$ \\
\hline HETATM & $46 \mathrm{C}$ & 0 & 2.070 & -0.267 & -3.148 & \\
\hline HETATM & $47 \mathrm{C}$ & 0 & 1.284 & 0.385 & -1.995 & $C$ \\
\hline HETATM & $48 \mathrm{C}$ & 0 & 1.690 & -0.147 & -0.604 & \\
\hline HETATM & $49 \mathrm{C}$ & 0 & -2.581 & -1.337 & -5.849 & C \\
\hline HETATM & $50 \mathrm{C}$ & 0 & 0.815 & 0.488 & 0.500 & $\mathrm{C}$ \\
\hline HETATM & $51 \mathrm{C}$ & 0 & 1.063 & -0.132 & 1.889 & $\mathrm{C}$ \\
\hline HETATM & $52 \mathrm{C}$ & 0 & -0.038 & 0.269 & 2.895 & $\mathrm{C}$ \\
\hline HETATM & $53 \mathrm{C}$ & 0 & 0.053 & -0.642 & 4.133 & $\mathrm{C}$ \\
\hline HETATM & $54 \mathrm{~N}$ & 0 & -1.116 & -0.406 & 5.032 & \\
\hline HETATM & $55 \mathrm{C}$ & 0 & -2.347 & -0.823 & 4.788 & C \\
\hline HETATM & $56 \mathrm{~N}$ & 0 & -3.142 & -0.375 & 5.752 & $\mathrm{~N}$ \\
\hline HETATM & $57 \mathrm{C}$ & 0 & -2.380 & 0.369 & 6.653 & $\mathrm{C}$ \\
\hline HETATM & $58 \mathrm{C}$ & 0 & -1.117 & 0.352 & 6.197 & C \\
\hline HETATM & $59 \mathrm{C}$ & 0 & -4.597 & -0.617 & 5.842 & \\
\hline HETATM & $60 \mathrm{H}$ & 0 & -5.058 & 0.245 & 6.298 & $\mathrm{H}$ \\
\hline HETATM & $61 \mathrm{H}$ & 0 & -4.787 & -1.492 & 6.444 & $\mathrm{H}$ \\
\hline HETATM & $62 \mathrm{H}$ & 0 & -4.971 & -0.742 & 4.842 & $\mathrm{H}$ \\
\hline HETATM & $63 \mathrm{H}$ & 0 & -2.382 & -2.302 & -5.418 & \\
\hline HETATM & $64 \mathrm{H}$ & 0 & -2.583 & -1.374 & -6.927 & \\
\hline HETATM & $65 \mathrm{H}$ & 0 & -3.521 & -0.975 & -5.477 & \\
\hline HETATM & $66 \mathrm{H}$ & 0 & -2.811 & 0.828 & 7.507 & $\mathrm{H}$ \\
\hline HETATM & $67 \mathrm{H}$ & 0 & -0.231 & 0.801 & 6.573 & $\mathrm{H}$ \\
\hline HETATM & $68 \mathrm{H}$ & 0 & -2.650 & -1.421 & 3.958 & $\mathrm{H}$ \\
\hline HETATM & $69 \mathrm{H}$ & 0 & 0.945 & -0.442 & 4.703 & $\mathrm{H}$ \\
\hline HETATM & $70 \mathrm{H}$ & 0 & 0.049 & -1.682 & 3.851 & $\mathrm{H}$ \\
\hline HETATM & $71 \mathrm{H}$ & 0 & -1.013 & 0.138 & 2.445 & $\mathrm{H}$ \\
\hline HETATM & $72 \mathrm{H}$ & 0 & 0.060 & 1.309 & 3.173 & $\mathrm{H}$ \\
\hline HETATM & $73 \mathrm{H}$ & 0 & 2.022 & 0.166 & 2.282 & $\mathrm{H}$ \\
\hline HETATM & $74 \mathrm{H}$ & 0 & 1.071 & -1.209 & 1.800 & $\mathrm{H}$ \\
\hline HETATM & $75 \mathrm{H}$ & 0 & -0.226 & 0.346 & 0.231 & $\mathrm{H}$ \\
\hline HETATM & $76 \mathrm{H}$ & 0 & 0.995 & 1.555 & 0.533 & 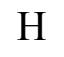 \\
\hline HETATM & $77 \mathrm{H}$ & 0 & 2.735 & 0.063 & -0.412 & $\mathrm{H}$ \\
\hline HETATM & $78 \mathrm{H}$ & 0 & 1.569 & -1.224 & -0.579 & $\mathrm{H}$ \\
\hline HETATM & $79 \mathrm{H}$ & 0 & 0.225 & 0.209 & -2.129 & $\mathrm{H}$ \\
\hline HETATM & $80 \mathrm{H}$ & 0 & 1.437 & 1.453 & -2.029 & 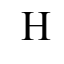 \\
\hline
\end{tabular}




\begin{tabular}{|c|c|c|c|c|c|c|}
\hline HETATM & $81 \mathrm{H}$ & 0 & 3.124 & -0.198 & -2.969 & $\mathrm{H}$ \\
\hline HETATM & $82 \mathrm{H}$ & 0 & 1.820 & -1.314 & -3.242 & $\mathrm{H}$ \\
\hline HETATM & $83 \mathrm{H}$ & 0 & 2.084 & 1.509 & -4.369 & \\
\hline HETATM & $84 \mathrm{H}$ & 0 & 2.387 & 0.035 & -5.281 & \\
\hline HETATM & $85 \mathrm{H}$ & 0 & 0.227 & -1.560 & -5.607 & \\
\hline HETATM & $86 \mathrm{H}$ & 0 & -0.292 & 2.312 & -4.092 & \\
\hline HETATM & $87 \mathrm{H}$ & 0 & -2.737 & 1.273 & -4.741 & \\
\hline HETATM & $88 \mathrm{~N}$ & 0 & -2.617 & 4.315 & 1.069 & \\
\hline HETATM & $89 \mathrm{O}$ & 0 & -2.808 & 3.066 & -3.257 & \\
\hline HETATM & $90 \mathrm{O}$ & 0 & -2.881 & 2.731 & 2.731 & \\
\hline HETATM & $91 \mathrm{C}$ & 0 & -5.278 & 2.167 & -2.119 & \\
\hline HETATM & $92 \mathrm{C}$ & 0 & -5.299 & 1.977 & 1.464 & \\
\hline НЕТАТМ & $93 \mathrm{C}$ & 0 & -4.655 & -2.684 & -2.264 & \\
\hline HETATM & $94 \mathrm{C}$ & 0 & -5.053 & -2.888 & 1.287 & \\
\hline HETATM & $95 \mathrm{C}$ & 0 & 0.206 & 5.635 & 0.007 & \\
\hline HETATM & $96 \mathrm{C}$ & 0 & 1.769 & 5.422 & 0.082 & $\mathrm{C}$ \\
\hline HETATM & $97 \mathrm{~N}$ & 0 & 2.040 & 4.629 & -1.102 & \\
\hline HETATM & $98 \mathrm{C}$ & 0 & 0.916 & 4.366 & -1.843 & \\
\hline HETATM & $99 \mathrm{~N}$ & 0 & -0.149 & 4.986 & -1.237 & \\
\hline HETATM & $100 \mathrm{~N}$ & 0 & -0.259 & 4.986 & 1.209 & \\
\hline HETATM & $101 \mathrm{C}$ & 0 & 0.755 & 4.447 & 1.968 & \\
\hline HETATM & $102 \mathrm{~N}$ & 0 & 1.941 & 4.747 & 1.343 & \\
\hline HETATM & $103 \mathrm{O}$ & 0 & 0.885 & 3.764 & -2.902 & \\
\hline HETATM & $104 \mathrm{O}$ & 0 & 0.625 & 3.897 & 3.040 & \\
\hline HETATM & $105 \mathrm{C}$ & 0 & 4.860 & 3.570 & 0.299 & \\
\hline HETATM & $106 \mathrm{C}$ & 0 & 5.752 & 2.266 & 0.400 & \\
\hline HETATM & $107 \mathrm{~N}$ & 0 & 5.479 & 1.589 & -0.849 & \\
\hline HETATM & $108 \mathrm{C}$ & 0 & 4.551 & 2.239 & -1.624 & \\
\hline HETATM & $109 \mathrm{~N}$ & 0 & 4.170 & 3.383 & -0.962 & \\
\hline HETATM & $110 \mathrm{~N}$ & 0 & 4.042 & 3.489 & 1.483 & \\
\hline HETATM & $111 \mathrm{C}$ & 0 & 4.225 & 2.326 & 2.194 & \\
\hline HETATM & $112 \mathrm{~N}$ & 0 & 5.242 & 1.628 & 1.591 & \\
\hline HETATM & $113 \mathrm{O}$ & 0 & 4.190 & 1.915 & -2.737 & \\
\hline HETATM & $114 \mathrm{O}$ & 0 & 3.652 & 2.017 & 3.215 & \\
\hline HETATM & $115 \mathrm{C}$ & 0 & 3.375 & 4.393 & -1.634 & \\
\hline HETATM & $116 \mathrm{C}$ & 0 & 3.213 & 4.551 & 2.010 & \\
\hline HETATM & $117 \mathrm{C}$ & 0 & -1.423 & 5.156 & -1.908 & \\
\hline HETATM & $118 \mathrm{C}$ & 0 & -1.598 & 5.093 & 1.747 & \\
\hline HETATM & $119 \mathrm{C}$ & 0 & 5.816 & 0.461 & 2.227 & \\
\hline HETATM & $120 \mathrm{C}$ & 0 & 6.201 & 0.421 & -1.331 & \\
\hline HETATM & $121 \mathrm{C}$ & 0 & 3.836 & -3.969 & 2.097 & \\
\hline HETATM & $122 \mathrm{C}$ & 0 & 4.260 & -4.058 & -1.506 & \\
\hline HETATM & $123 \mathrm{C}$ & 0 & 5.451 & -2.853 & 0.441 & \\
\hline HETATM & $124 \mathrm{C}$ & 0 & 6.098 & -1.407 & 0.490 & \\
\hline HETATM & $125 \mathrm{~N}$ & 0 & 5.753 & -0.858 & -0.806 & \\
\hline HETATM & $126 \mathrm{~N}$ & 0 & 4.864 & -2.896 & -0.882 & \\
\hline
\end{tabular}




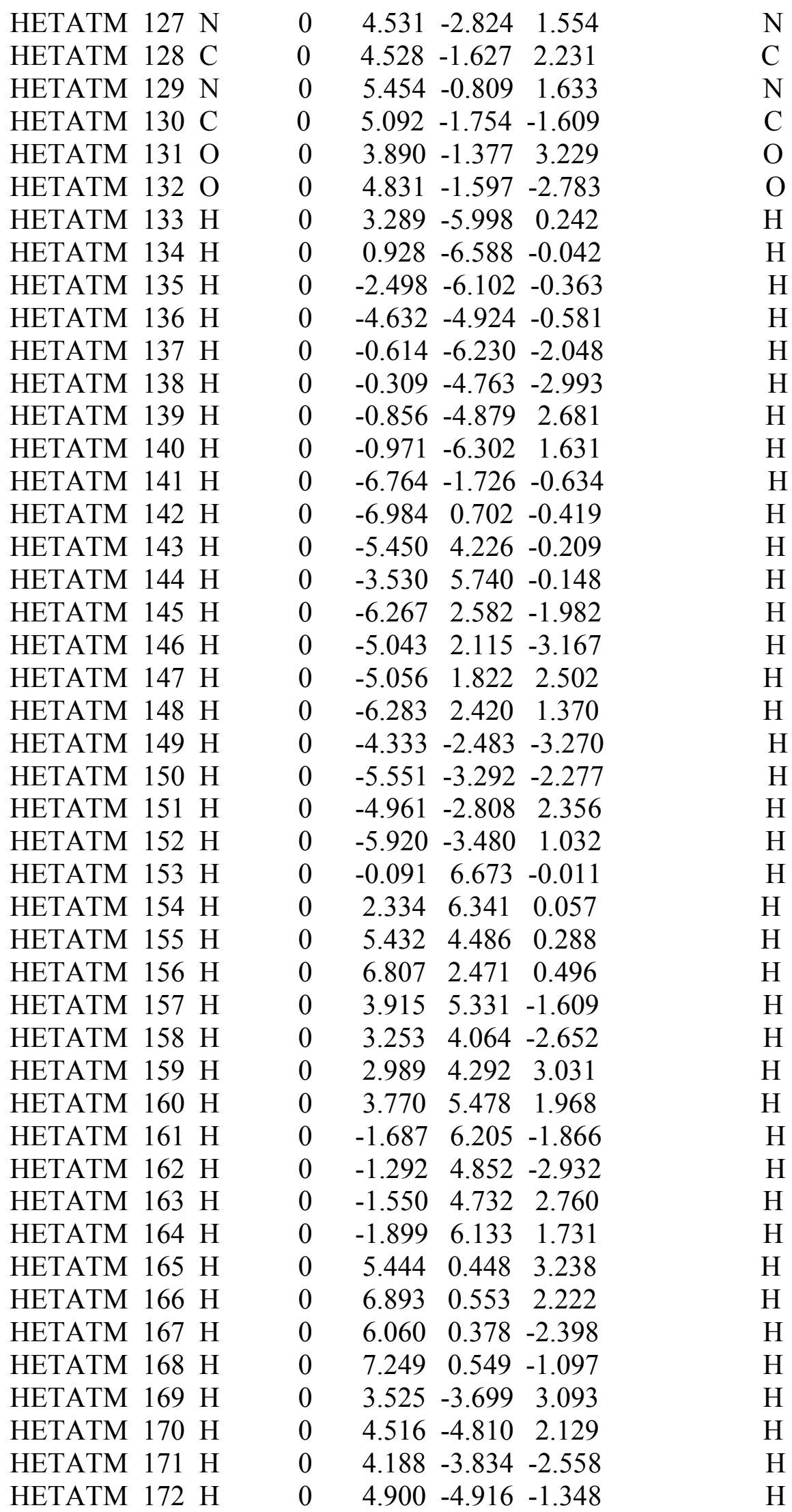




$\begin{array}{llllllll}\text { HETATM } & 173 \mathrm{H} & 0 & 6.170 & -3.649 & 0.563 & \mathrm{H} \\ \text { HETATM } & 174 \mathrm{H} & 0 & 7.169 & -1.421 & 0.621 & \mathrm{H}\end{array}$

END

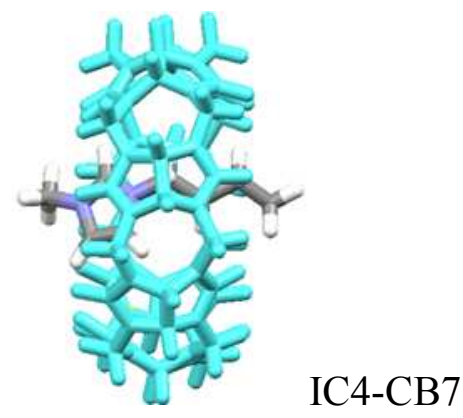

SCF Done: $E=-4583.14322446$

REMARK 1 File created by GaussView 5.0.8

\begin{tabular}{|c|c|c|c|c|c|c|}
\hline HETATM & $1 \mathrm{C}$ & 0 & 5.274 & -2.779 & 0.044 & $\mathrm{C}$ \\
\hline HETATM & $2 \mathrm{C}$ & 0 & 4.311 & -4.024 & 0.222 & $\mathrm{C}$ \\
\hline HETATM & $3 \mathrm{~N}$ & 0 & 3.589 & -4.051 & -1.031 & $\mathrm{~N}$ \\
\hline HETATM & $4 \mathrm{C}$ & 0 & 3.773 & -2.915 & -1.767 & $\mathrm{C}$ \\
\hline HETATM & $5 \mathrm{~N}$ & 0 & 4.784 & -2.196 & -1.190 & $\mathrm{~N}$ \\
\hline HETATM & $6 \mathrm{~N}$ & 0 & 5.030 & -2.004 & 1.233 & $\mathrm{~N}$ \\
\hline HETATM & $7 \mathrm{C}$ & 0 & 3.983 & -2.489 & 1.983 & $\mathrm{C}$ \\
\hline HETATM & $8 \mathrm{~N}$ & 0 & 3.529 & -3.641 & 1.379 & $\mathrm{~N}$ \\
\hline HETATM & $9 \mathrm{O}$ & 0 & 3.182 & -2.615 & -2.790 & $\mathrm{O}$ \\
\hline HETATM & $10 \mathrm{O}$ & 0 & 3.585 & -2.031 & 3.029 & $\mathrm{O}$ \\
\hline HETATM & $11 \mathrm{C}$ & 0 & 1.055 & -5.629 & 0.334 & $\mathrm{C}$ \\
\hline HETATM & $12 \mathrm{C}$ & 0 & -0.523 & -5.691 & 0.265 & $\mathrm{C}$ \\
\hline HETATM & $13 \mathrm{~N}$ & 0 & -0.800 & -5.125 & -1.041 & $\mathrm{~N}$ \\
\hline HETATM & $14 \mathrm{C}$ & 0 & 0.330 & -4.832 & -1.759 & $\mathrm{C}$ \\
\hline HETATM & $15 \mathrm{~N}$ & 0 & 1.413 & -5.185 & -0.991 & $\mathrm{~N}$ \\
\hline HETATM & $16 \mathrm{~N}$ & 0 & 1.290 & -4.679 & 1.399 & $\mathrm{~N}$ \\
\hline HETATM & $17 \mathrm{C}$ & 0 & 0.123 & -4.242 & 2.001 & $\mathrm{C}$ \\
\hline HETATM & $18 \mathrm{~N}$ & 0 & -0.927 & -4.895 & 1.393 & $\mathrm{~N}$ \\
\hline HETATM & $19 \mathrm{O}$ & 0 & 0.368 & -4.420 & -2.900 & $\mathrm{O}$ \\
\hline HETATM & $20 \mathrm{O}$ & 0 & 0.049 & -3.498 & 2.950 & $\mathrm{O}$ \\
\hline HETATM & $21 \mathrm{C}$ & 0 & 2.758 & -5.139 & -1.514 & $\mathrm{C}$ \\
\hline HETATM & $22 \mathrm{C}$ & 0 & 2.569 & -4.491 & 2.061 & $\mathrm{C}$ \\
\hline HETATM & $23 \mathrm{C}$ & 0 & -3.865 & -4.378 & 0.043 & $\mathrm{C}$ \\
\hline HETATM & $24 \mathrm{C}$ & 0 & -4.972 & -3.257 & -0.064 & $\mathrm{C}$ \\
\hline HETATM & $25 \mathrm{~N}$ & 0 & -4.499 & -2.459 & -1.181 & $\mathrm{~N}$ \\
\hline HETATM & $26 \mathrm{C}$ & 0 & -3.390 & -2.990 & -1.794 & $\mathrm{C}$ \\
\hline HETATM & $27 \mathrm{~N}$ & 0 & -3.055 & -4.144 & -1.130 & $\mathrm{~N}$ \\
\hline HETATM & $28 \mathrm{~N}$ & 0 & -3.247 & -4.084 & 1.315 & $\mathrm{~N}$ \\
\hline HETATM & $29 \mathrm{C}$ & 0 & -3.901 & -3.099 & 2.024 & $\mathrm{C}$ \\
\hline HETATM & $30 \mathrm{~N}$ & 0 & -4.930 & -2.636 & 1.233 & $\mathrm{~N}$ \\
\hline
\end{tabular}




\begin{tabular}{|c|c|c|c|c|c|c|}
\hline HETATM & 310 & 0 & -2.866 & -2.567 & -2.806 & \\
\hline HETATM & $32 \mathrm{O}$ & 0 & -3.674 & -2.776 & 3.164 & $\Omega$ \\
\hline HETATM & $33 \mathrm{C}$ & 0 & -6.047 & 0.251 & -0.014 & \\
\hline HETATM & $34 \mathrm{C}$ & 0 & -5.724 & 1.792 & 0.129 & \\
\hline HETATM & $35 \mathrm{~N}$ & 0 & -5.017 & 2.090 & -1.094 & \\
\hline HETATM & $36 \mathrm{C}$ & 0 & -4.682 & 0.964 & -1.799 & \\
\hline HETATM & $37 \mathrm{~N}$ & 0 & -5.248 & -0.116 & -1.171 & \\
\hline HETATM & $38 \mathrm{~N}$ & 0 & -5.625 & -0.287 & 1.250 & \\
\hline HETATM & $39 \mathrm{C}$ & 0 & -4.925 & 0.620 & 2.015 & \\
\hline HETATM & $40 \mathrm{~N}$ & 0 & -0.096 & -0.675 & -2.911 & \\
\hline HETATM & $41 \mathrm{C}$ & 0 & -1.178 & 0.095 & -2.500 & \\
\hline HETATM & $42 \mathrm{C}$ & 0 & -0.823 & 0.665 & -1.337 & \\
\hline HETATM & $43 \mathrm{~N}$ & 0 & 0.474 & 0.233 & -1.043 & \\
\hline HETATM & $44 \mathrm{C}$ & 0 & 0.879 & -0.557 & -2.027 & \\
\hline HETATM & $45 \mathrm{C}$ & 0 & 1.222 & 0.553 & 0.205 & $\mathrm{C}$ \\
\hline HETATM & $46 \mathrm{C}$ & 0 & 0.387 & 0.201 & 1.457 & $\mathrm{C}$ \\
\hline HETATM & $47 \mathrm{C}$ & 0 & 1.179 & 0.423 & 2.768 & \\
\hline HETATM & $48 \mathrm{C}$ & 0 & 0.285 & 0.064 & 3.981 & \\
\hline HETATM & $49 \mathrm{C}$ & 0 & -0.056 & -1.544 & -4.115 & \\
\hline HETATM & $50 \mathrm{H}$ & 0 & 0.833 & 0.217 & 4.901 & \\
\hline HETATM & $51 \mathrm{H}$ & 0 & -0.018 & -0.973 & 3.929 & \\
\hline HETATM & $52 \mathrm{H}$ & 0 & -0.594 & 0.695 & 3.995 & \\
\hline HETATM & $53 \mathrm{H}$ & 0 & 1.491 & 1.453 & 2.846 & $\mathrm{H}$ \\
\hline HETATM & $54 \mathrm{H}$ & 0 & 2.060 & -0.201 & 2.784 & \\
\hline HETATM & $55 \mathrm{H}$ & 0 & 0.079 & -0.834 & 1.394 & $\mathrm{H}$ \\
\hline HETATM & $56 \mathrm{H}$ & 0 & -0.498 & 0.820 & 1.490 & $\mathrm{H}$ \\
\hline HETATM & $57 \mathrm{H}$ & 0 & 1.477 & 1.600 & 0.184 & $\mathrm{H}$ \\
\hline HETATM & $58 \mathrm{H}$ & 0 & 2.133 & -0.026 & 0.182 & \\
\hline HETATM & $59 \mathrm{H}$ & 0 & 0.809 & -2.180 & -4.038 & \\
\hline HETATM & $60 \mathrm{H}$ & 0 & -0.006 & -0.922 & -4.994 & \\
\hline HETATM & $61 \mathrm{H}$ & 0 & -0.947 & -2.145 & -4.104 & \\
\hline HETATM & $62 \mathrm{H}$ & 0 & 1.828 & -1.020 & -2.139 & \\
\hline HETATM & $63 \mathrm{H}$ & 0 & -1.355 & 1.319 & -0.694 & \\
\hline HETATM & $64 \mathrm{H}$ & 0 & -2.089 & 0.154 & -3.041 & $\mathrm{H}$ \\
\hline HETATM & $65 \mathrm{~N}$ & 0 & -4.934 & 1.822 & 1.341 & $\mathrm{~N}$ \\
\hline HETATM & $66 \mathrm{O}$ & 0 & -4.064 & 0.936 & -2.846 & \\
\hline HETATM & $67 \mathrm{O}$ & 0 & -4.467 & 0.424 & 3.114 & \\
\hline HETATM & $68 \mathrm{C}$ & 0 & -5.273 & -1.413 & -1.819 & \\
\hline HETATM & $69 \mathrm{C}$ & 0 & -5.858 & -1.637 & 1.724 & \\
\hline HETATM & $70 \mathrm{C}$ & 0 & -2.105 & -5.092 & -1.674 & \\
\hline HETATM & $71 \mathrm{C}$ & 0 & -2.254 & -4.907 & 1.977 & \\
\hline HETATM & $72 \mathrm{C}$ & 0 & -3.499 & 4.648 & 0.287 & \\
\hline HETATM & $73 \mathrm{C}$ & 0 & -2.099 & 5.383 & 0.275 & 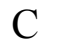 \\
\hline HETATM & $74 \mathrm{~N}$ & 0 & -1.630 & 5.154 & -1.071 & \\
\hline HETATM & $75 \mathrm{C}$ & 0 & -2.484 & 4.386 & -1.828 & 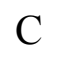 \\
\hline HETATM & $76 \mathrm{~N}$ & 0 & -3.569 & 4.075 & -1.039 & \\
\hline
\end{tabular}




\begin{tabular}{|c|c|c|c|c|c|c|}
\hline HETATM & $77 \mathrm{~N}$ & 0 & -3.360 & 3.707 & 1.369 & $\mathrm{~N}$ \\
\hline HETATM & $78 \mathrm{C}$ & 0 & -2.099 & 3.696 & 1.923 & $\mathrm{C}$ \\
\hline HETATM & $79 \mathrm{~N}$ & 0 & -1.373 & 4.705 & 1.324 & $\mathbf{N}$ \\
\hline HETATM & $80 \mathrm{O}$ & 0 & -2.352 & 4.109 & -2.998 & $\mathrm{O}$ \\
\hline HETATM & 810 & 0 & -1.723 & 2.989 & 2.830 & $\mathrm{O}$ \\
\hline HETATM & $82 \mathrm{C}$ & 0 & 1.502 & 5.598 & 0.028 & $\mathrm{C}$ \\
\hline HETATM & $83 \mathrm{C}$ & 0 & 2.951 & 5.005 & -0.169 & $\mathrm{C}$ \\
\hline HETATM & $84 \mathrm{~N}$ & 0 & 2.762 & 4.129 & -1.308 & $\mathrm{~N}$ \\
\hline HETATM & $85 \mathrm{C}$ & 0 & 1.535 & 4.293 & -1.921 & $\mathrm{C}$ \\
\hline НЕТАТМ & $86 \mathrm{~N}$ & 0 & 0.823 & 5.207 & -1.180 & $\mathrm{~N}$ \\
\hline HETATM & $87 \mathrm{~N}$ & 0 & 1.074 & 4.936 & 1.245 & $\mathrm{~N}$ \\
\hline HETATM & $88 \mathrm{C}$ & 0 & 2.094 & 4.275 & 1.887 & $\mathrm{C}$ \\
\hline HETATM & $89 \mathrm{~N}$ & 0 & 3.212 & 4.354 & 1.090 & $\mathrm{~N}$ \\
\hline HETATM & $90 \mathrm{O}$ & 0 & 1.192 & 3.793 & -2.966 & 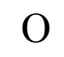 \\
\hline HETATM & 910 & 0 & 2.041 & 3.782 & 2.991 & $\mathrm{O}$ \\
\hline HETATM & $92 \mathrm{C}$ & 0 & -0.440 & 5.742 & -1.653 & $\mathrm{C}$ \\
\hline HETATM & $93 \mathrm{C}$ & 0 & -0.165 & 5.211 & 1.947 & $\mathrm{C}$ \\
\hline HETATM & $94 \mathrm{C}$ & 0 & -4.732 & 3.420 & -1.602 & $\mathrm{C}$ \\
\hline HETATM & $95 \mathrm{C}$ & 0 & -4.466 & 3.020 & 2.007 & $\mathrm{C}$ \\
\hline HETATM & $96 \mathrm{C}$ & 0 & 4.496 & 3.852 & 1.542 & $\mathrm{C}$ \\
\hline HETATM & $97 \mathrm{C}$ & 0 & 3.840 & 3.478 & -2.022 & $\mathrm{C}$ \\
\hline HETATM & $98 \mathrm{C}$ & 0 & 5.805 & -0.857 & 1.679 & $\mathrm{C}$ \\
\hline HETATM & $99 \mathrm{C}$ & 0 & 5.409 & -1.094 & -1.896 & $\mathrm{C}$ \\
\hline HETATM & $100 \mathrm{C}$ & 0 & 5.882 & 0.828 & -0.263 & $C$ \\
\hline HETATM & $101 \mathrm{C}$ & 0 & 5.399 & 2.334 & -0.324 & $\mathrm{C}$ \\
\hline HETATM & $102 \mathrm{~N}$ & 0 & 4.408 & 2.306 & -1.382 & N \\
\hline HETATM & $103 \mathrm{~N}$ & 0 & 5.158 & 0.221 & -1.354 & $\mathbf{N}$ \\
\hline HETATM & $104 \mathrm{~N}$ & 0 & 5.474 & 0.415 & 1.060 & $\mathrm{~N}$ \\
\hline HETATM & $105 \mathrm{C}$ & 0 & 4.928 & 1.440 & 1.806 & $\mathrm{C}$ \\
\hline HETATM & $106 \mathrm{~N}$ & 0 & 4.896 & 2.563 & 1.007 & $\mathrm{~N}$ \\
\hline HETATM & $107 \mathrm{C}$ & 0 & 4.240 & 1.062 & -1.931 & $\mathrm{C}$ \\
\hline HETATM & $108 \mathrm{O}$ & 0 & 4.617 & 1.384 & 2.971 & $\mathrm{O}$ \\
\hline HETATM & $109 \mathrm{O}$ & 0 & 3.470 & 0.763 & -2.820 & $\mathrm{O}$ \\
\hline HETATM & $110 \mathrm{H}$ & 0 & 6.317 & -3.042 & -0.043 & $\mathrm{H}$ \\
\hline HETATM & $111 \mathrm{H}$ & 0 & 4.827 & -4.957 & 0.387 & $\mathrm{H}$ \\
\hline HETATM & $112 \mathrm{H}$ & 0 & 1.517 & -6.579 & 0.557 & $\mathrm{H}$ \\
\hline HETATM & $113 \mathrm{H}$ & 0 & -0.921 & -6.692 & 0.339 & \\
\hline HETATM & $114 \mathrm{H}$ & 0 & 3.258 & -6.069 & -1.283 & \\
\hline HETATM & $115 \mathrm{H}$ & 0 & 2.667 & -5.017 & -2.580 & \\
\hline HETATM & $116 \mathrm{H}$ & 0 & 2.366 & -4.016 & 3.007 & $\mathrm{H}$ \\
\hline HETATM & $117 \mathrm{H}$ & 0 & 3.018 & -5.465 & 2.220 & $\mathrm{H}$ \\
\hline HETATM & $118 \mathrm{H}$ & 0 & -4.265 & -5.381 & 0.028 & \\
\hline HETATM & $119 \mathrm{H}$ & 0 & -5.959 & -3.642 & -0.272 & \\
\hline HETATM & $120 \mathrm{H}$ & 0 & -7.091 & 0.042 & -0.194 & l \\
\hline HETATM & $121 \mathrm{H}$ & 0 & -6.603 & 2.412 & 0.220 & $\mathrm{H}$ \\
\hline HETATM & $122 \mathrm{H}$ & 0 & -6.302 & -1.744 & -1.889 & \\
\hline
\end{tabular}




\begin{tabular}{|c|c|c|c|c|}
\hline HETATM $123 \mathrm{H}$ & 0 & -4.862 & -1.274 & -2.805 \\
\hline HETATM $124 \mathrm{H}$ & 0 & -5.742 & -1.615 & 2.794 \\
\hline HETATM $125 \mathrm{H}$ & 0 & -6.863 & -1.930 & 1.456 \\
\hline HETATM $126 \mathrm{H}$ & 0 & -1.934 & -4.825 & -2.703 \\
\hline HETATM $127 \mathrm{H}$ & 0 & -2.547 & -6.079 & -1.614 \\
\hline HETATM $128 \mathrm{H}$ & 0 & -2.170 & -4.520 & 2.979 \\
\hline HETATM $129 \mathrm{H}$ & 0 & -2.598 & -5.934 & 1.999 \\
\hline HETATM $130 \mathrm{H}$ & 0 & -4.334 & 5.311 & 0.463 \\
\hline HETATM $131 \mathrm{H}$ & 0 & -2.164 & 6.441 & 0.482 \\
\hline HETATM $132 \mathrm{H}$ & 0 & 1.486 & 6.671 & 0.146 \\
\hline HETATM $133 \mathrm{H}$ & 0 & 3.702 & 5.753 & -0.378 \\
\hline HETATM $134 \mathrm{H}$ & 0 & -0.445 & 6.808 & -1.467 \\
\hline HETATM $135 \mathrm{H}$ & 0 & -0.494 & 5.544 & -2.710 \\
\hline HETATM $136 \mathrm{H}$ & 0 & -0.075 & 4.736 & 2.910 \\
\hline HETATM $137 \mathrm{H}$ & 0 & -0.272 & 6.283 & 2.067 \\
\hline HETATM $138 \mathrm{H}$ & 0 & -5.606 & 4.035 & -1.432 \\
\hline HETATM $139 \mathrm{H}$ & 0 & -4.540 & 3.325 & -2.658 \\
\hline HETATM $140 \mathrm{H}$ & 0 & -4.132 & 2.706 & 2.982 \\
\hline HETATM $141 \mathrm{H}$ & 0 & -5.287 & 3.721 & 2.100 \\
\hline HETATM $142 \mathrm{H}$ & 0 & 4.423 & 3.727 & 2.610 \\
\hline HETATM $143 \mathrm{H}$ & 0 & 5.255 & 4.583 & 1.296 \\
\hline HETATM $144 \mathrm{H}$ & 0 & 3.428 & 3.156 & -2.965 \\
\hline HETATM $145 \mathrm{H}$ & 0 & 4.638 & 4.192 & -2.186 \\
\hline HETATM $146 \mathrm{H}$ & 0 & 5.609 & -0.737 & 2.732 \\
\hline HETATM $147 \mathrm{H}$ & 0 & 6.852 & -1.071 & 1.512 \\
\hline HETATM $148 \mathrm{H}$ & 0 & 5.009 & -1.107 & -2.896 \\
\hline HETATM $149 \mathrm{H}$ & 0 & 6.478 & -1.258 & -1.916 \\
\hline HETATM $150 \mathrm{H}$ & 0 & 6.946 & 0.707 & -0.397 \\
\hline HETATM $151 \mathrm{H}$ & 0 & 6.187 & 3.032 & -0.564 \\
\hline
\end{tabular}

END

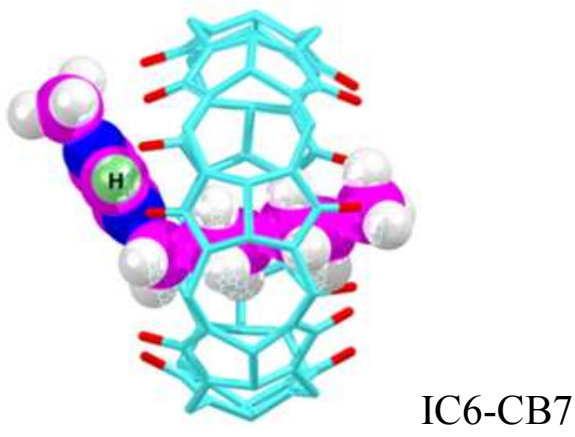

SCF Done: $E=-4660.61830160$

REMARK 1 File created by GaussView 5.0.8

$\begin{array}{llllllll}\text { HETATM } & 1 & \mathrm{C} & 0 & -5.644 & -1.712 & -0.385 & \mathrm{C} \\ \text { HETATM } & 2 & \mathrm{C} & 0 & -4.930 & -3.120 & -0.494 & \mathrm{C} \\ \text { HETATM } & 3 \mathrm{~N} & 0 & -4.190 & -3.204 & 0.749 & \mathrm{~N}\end{array}$




\begin{tabular}{|c|c|c|c|c|c|c|}
\hline HETATM & $4 \mathrm{C}$ & 0 & -4.254 & -2.054 & 1.487 & $\mathrm{C}$ \\
\hline HETATM & $5 \mathrm{~N}$ & 0 & -5.086 & -1.179 & 0.844 & $\mathrm{~N}$ \\
\hline HETATM & $6 \mathrm{~N}$ & 0 & -5.252 & -1.054 & -1.601 & $\mathrm{~N}$ \\
\hline HETATM & $7 \mathrm{C}$ & 0 & -4.356 & -1.785 & -2.352 & $\mathrm{C}$ \\
\hline HETATM & $8 \mathrm{~N}$ & 0 & -4.147 & -2.982 & -1.696 & $\mathrm{~N}$ \\
\hline HETATM & $9 \mathrm{O}$ & 0 & -3.746 & -1.870 & 2.579 & $\mathrm{O}$ \\
\hline HETATM & $10 \mathrm{O}$ & 0 & -3.917 & -1.480 & -3.432 & $\mathrm{O}$ \\
\hline HETATM & $11 \mathrm{C}$ & 0 & -2.082 & -5.353 & -0.599 & $\mathrm{C}$ \\
\hline HETATM & $12 \mathrm{C}$ & 0 & -0.545 & -5.724 & -0.583 & $\mathrm{C}$ \\
\hline HETATM & $13 \mathrm{~N}$ & 0 & -0.132 & -5.320 & 0.745 & $\mathrm{~N}$ \\
\hline HETATM & $14 \mathrm{C}$ & 0 & -1.144 & -4.764 & 1.482 & $\mathrm{C}$ \\
\hline HETATM & $15 \mathrm{~N}$ & 0 & -2.279 & -4.760 & 0.709 & $\mathrm{~N}$ \\
\hline HETATM & $16 \mathrm{~N}$ & 0 & -2.189 & -4.467 & -1.728 & $\mathrm{~N}$ \\
\hline HETATM & $17 \mathrm{C}$ & 0 & -0.980 & -4.225 & -2.347 & $\mathrm{C}$ \\
\hline HETATM & $18 \mathrm{~N}$ & 0 & -0.019 & -4.956 & -1.684 & $\mathrm{~N}$ \\
\hline HETATM & $19 \mathrm{O}$ & 0 & -1.073 & -4.433 & 2.650 & $\mathrm{O}$ \\
\hline HETATM & $20 \mathrm{O}$ & 0 & -0.818 & -3.580 & -3.355 & $\mathrm{O}$ \\
\hline HETATM & $21 \mathrm{C}$ & 0 & -3.572 & -4.413 & 1.264 & $\mathrm{C}$ \\
\hline HETATM & $22 \mathrm{C}$ & 0 & -3.432 & -4.059 & -2.352 & $\mathrm{C}$ \\
\hline HETATM & $23 \mathrm{C}$ & 0 & 3.003 & -5.013 & -0.345 & $\mathrm{C}$ \\
\hline HETATM & $24 \mathrm{C}$ & 0 & 4.258 & -4.067 & -0.171 & $\mathrm{C}$ \\
\hline HETATM & $25 \mathrm{~N}$ & 0 & 3.960 & -3.382 & 1.071 & $\mathrm{~N}$ \\
\hline HETATM & $26 \mathrm{C}$ & 0 & 2.821 & -3.836 & 1.686 & $\mathrm{C}$ \\
\hline HETATM & $27 \mathrm{~N}$ & 0 & 2.270 & -4.806 & 0.886 & $\mathrm{~N}$ \\
\hline HETATM & $28 \mathrm{~N}$ & 0 & 2.394 & -4.507 & -1.552 & $\mathrm{~N}$ \\
\hline HETATM & $29 \mathrm{C}$ & 0 & 3.132 & -3.513 & -2.159 & $\mathrm{C}$ \\
\hline HETATM & $30 \mathrm{~N}$ & 0 & 4.234 & -3.269 & -1.369 & $\mathrm{~N}$ \\
\hline HETATM & $31 \mathrm{O}$ & 0 & 2.431 & -3.511 & 2.790 & $\mathrm{O}$ \\
\hline HETATM & $32 \mathrm{O}$ & 0 & 2.907 & -3.020 & -3.238 & $\mathrm{O}$ \\
\hline HETATM & $33 \mathrm{C}$ & 0 & 5.882 & -0.815 & 0.098 & $\mathrm{C}$ \\
\hline HETATM & $34 \mathrm{C}$ & 0 & 5.883 & 0.768 & 0.148 & $\mathrm{C}$ \\
\hline HETATM & $35 \mathrm{~N}$ & 0 & 5.002 & 1.051 & 1.262 & $\mathrm{~N}$ \\
\hline HETATM & $36 \mathrm{C}$ & 0 & 4.477 & -0.077 & 1.834 & $\mathrm{C}$ \\
\hline HETATM & $37 \mathrm{~N}$ & 0 & 5.018 & -1.167 & 1.206 & $\mathrm{~N}$ \\
\hline HETATM & $38 \mathrm{~N}$ & 0 & 5.369 & -1.092 & -1.219 & $\mathrm{~N}$ \\
\hline HETATM & $39 \mathrm{C}$ & 0 & 5.084 & 0.044 & -1.948 & $\mathrm{C}$ \\
\hline HETATM & $40 \mathrm{~N}$ & 0 & -1.108 & -1.598 & 4.172 & $\mathrm{~N}$ \\
\hline HETATM & $41 \mathrm{C}$ & 0 & 0.246 & -1.845 & 3.959 & $\mathrm{C}$ \\
\hline HETATM & $42 \mathrm{C}$ & 0 & 0.742 & -0.780 & 3.308 & $\mathrm{C}$ \\
\hline HETATM & $43 \mathrm{~N}$ & 0 & -0.323 & 0.096 & 3.107 & $\mathrm{~N}$ \\
\hline HETATM & $44 \mathrm{C}$ & 0 & -1.419 & -0.431 & 3.630 & $\mathrm{C}$ \\
\hline HETATM & $45 \mathrm{C}$ & 0 & -0.276 & 1.410 & 2.412 & $\mathrm{C}$ \\
\hline HETATM & $46 \mathrm{C}$ & 0 & -0.585 & 1.256 & 0.906 & $\mathrm{C}$ \\
\hline HETATM & $47 \mathrm{C}$ & 0 & 0.568 & 0.669 & 0.063 & $\mathrm{C}$ \\
\hline HETATM & $48 \mathrm{C}$ & 0 & 0.155 & 0.459 & -1.416 & $\mathrm{C}$ \\
\hline HETATM & $49 \mathrm{C}$ & 0 & -2.019 & -2.506 & 4.896 & $\mathrm{C}$ \\
\hline
\end{tabular}




\begin{tabular}{|c|c|c|c|c|c|c|}
\hline HETATM & $50 \mathrm{C}$ & 0 & 1.340 & -0.002 & -2.295 & $\mathrm{C}$ \\
\hline HETATM & $51 \mathrm{C}$ & 0 & 0.887 & -0.445 & -3.705 & $\mathrm{C}$ \\
\hline HETATM & $52 \mathrm{H}$ & 0 & 1.751 & -0.725 & -4.290 & $\mathrm{H}$ \\
\hline HETATM & $53 \mathrm{H}$ & 0 & 0.371 & 0.365 & -4.204 & $\mathrm{H}$ \\
\hline HETATM & $54 \mathrm{H}$ & 0 & 0.228 & -1.300 & -3.649 & \\
\hline HETATM & $55 \mathrm{H}$ & 0 & 1.847 & -0.829 & -1.816 & $\mathrm{H}$ \\
\hline HETATM & $56 \mathrm{H}$ & 0 & 2.044 & 0.809 & -2.400 & $\mathrm{H}$ \\
\hline HETATM & $57 \mathrm{H}$ & 0 & -0.245 & 1.377 & -1.828 & \\
\hline HETATM & $58 \mathrm{H}$ & 0 & -0.634 & -0.284 & -1.456 & $\mathrm{H}$ \\
\hline HETATM & $59 \mathrm{H}$ & 0 & 0.878 & -0.282 & 0.479 & $\mathrm{H}$ \\
\hline HETATM & $60 \mathrm{H}$ & 0 & 1.420 & 1.338 & 0.112 & $\mathrm{H}$ \\
\hline HETATM & $61 \mathrm{H}$ & 0 & -0.833 & 2.236 & 0.519 & $\mathrm{H}$ \\
\hline HETATM & $62 \mathrm{H}$ & 0 & -1.467 & 0.640 & 0.799 & $\mathrm{H}$ \\
\hline HETATM & $63 \mathrm{H}$ & 0 & 0.695 & 1.836 & 2.592 & $\mathrm{H}$ \\
\hline HETATM & $64 \mathrm{H}$ & 0 & -1.010 & 2.037 & 2.879 & $\mathrm{H}$ \\
\hline HETATM & $65 \mathrm{H}$ & 0 & -3.029 & -2.183 & 4.724 & $\mathrm{H}$ \\
\hline HETATM & $66 \mathrm{H}$ & 0 & -1.779 & -2.481 & 5.948 & $\mathrm{H}$ \\
\hline HETATM & $67 \mathrm{H}$ & 0 & -1.881 & -3.491 & 4.490 & $\mathrm{H}$ \\
\hline HETATM & $68 \mathrm{H}$ & 0 & -2.383 & 0.012 & 3.575 & $\mathrm{H}$ \\
\hline HETATM & $69 \mathrm{H}$ & 0 & 1.736 & -0.554 & 3.007 & $\mathrm{H}$ \\
\hline HETATM & $70 \mathrm{H}$ & 0 & 0.711 & -2.757 & 4.229 & $\mathrm{H}$ \\
\hline HETATM & $71 \mathrm{~N}$ & 0 & 5.392 & 1.130 & -1.157 & $\mathrm{~N}$ \\
\hline HETATM & $72 \mathrm{O}$ & 0 & 3.739 & -0.106 & 2.801 & $\mathrm{O}$ \\
\hline HETATM & $73 \mathrm{O}$ & 0 & 4.728 & 0.080 & -3.101 & $\mathrm{O}$ \\
\hline HETATM & $74 \mathrm{C}$ & 0 & 4.876 & -2.498 & 1.762 & $\mathrm{C}$ \\
\hline HETATM & $75 \mathrm{C}$ & 0 & 5.303 & -2.404 & -1.832 & $\mathrm{C}$ \\
\hline HETATM & $76 \mathrm{C}$ & 0 & 1.158 & -5.617 & 1.339 & $\mathrm{C}$ \\
\hline HETATM & $77 \mathrm{C}$ & 0 & 1.300 & -5.137 & -2.262 & $\mathrm{C}$ \\
\hline HETATM & $78 \mathrm{C}$ & 0 & 4.325 & 4.055 & 0.061 & $\mathrm{C}$ \\
\hline HETATM & $79 \mathrm{C}$ & 0 & 3.093 & 5.039 & -0.068 & $\mathrm{C}$ \\
\hline HETATM & $80 \mathrm{~N}$ & 0 & 2.369 & 4.815 & 1.161 & $\mathrm{~N}$ \\
\hline HETATM & $81 \mathrm{C}$ & 0 & 2.847 & 3.744 & 1.873 & $\mathrm{C}$ \\
\hline HETATM & $82 \mathrm{~N}$ & 0 & 3.977 & 3.288 & 1.242 & $\mathrm{~N}$ \\
\hline HETATM & $83 \mathrm{~N}$ & 0 & 4.300 & 3.337 & -1.186 & $\mathrm{~N}$ \\
\hline HETATM & $84 \mathrm{C}$ & 0 & 3.172 & 3.597 & -1.933 & $\mathrm{C}$ \\
\hline HETATM & $85 \mathrm{~N}$ & 0 & 2.451 & 4.569 & -1.274 & $\mathrm{~N}$ \\
\hline HETATM & $86 \mathrm{O}$ & 0 & 2.401 & 3.327 & 2.922 & $\mathrm{O}$ \\
\hline HETATM & $87 \mathrm{O}$ & 0 & 2.912 & 3.126 & -3.015 & $\mathrm{O}$ \\
\hline HETATM & $88 \mathrm{C}$ & 0 & -0.436 & 5.868 & -0.210 & $\mathrm{C}$ \\
\hline HETATM & $89 \mathrm{C}$ & 0 & -1.987 & 5.549 & -0.205 & $\mathrm{C}$ \\
\hline HETATM & $90 \mathrm{~N}$ & 0 & -2.154 & 4.846 & 1.053 & $\mathrm{~N}$ \\
\hline HETATM & $91 \mathrm{C}$ & 0 & -1.010 & 4.815 & 1.813 & 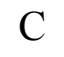 \\
\hline HETATM & $92 \mathrm{~N}$ & 0 & -0.014 & 5.434 & 1.102 & $\mathrm{~N}$ \\
\hline HETATM & $93 \mathrm{~N}$ & 0 & 0.043 & 5.083 & -1.325 & $\mathrm{~N}$ \\
\hline HETATM & $94 \mathrm{C}$ & 0 & -0.966 & 4.429 & -2.002 & $\mathrm{C}$ \\
\hline HETATM & $95 \mathrm{~N}$ & 0 & -2.158 & 4.759 & -1.393 & \\
\hline
\end{tabular}




\begin{tabular}{|c|c|c|c|c|c|c|}
\hline HETATM & $96 \mathrm{O}$ & 0 & -0.924 & 4.384 & 2.945 & $\Omega$ \\
\hline HETATM & $97 \mathrm{O}$ & 0 & -0.839 & 3.766 & -3.003 & $\Omega$ \\
\hline HETATM & $98 \mathrm{C}$ & 0 & 1.300 & 5.649 & 1.678 & $\mathrm{C}$ \\
\hline HETATM & $99 \mathrm{C}$ & 0 & 1.349 & 5.233 & -1.941 & $\mathrm{C}$ \\
\hline HETATM & $100 \mathrm{C}$ & 0 & 4.856 & 2.344 & 1.902 & \\
\hline HETATM & $101 \mathrm{C}$ & 0 & 5.352 & 2.477 & -1.694 & \\
\hline HETATM & $102 \mathrm{C}$ & 0 & -3.428 & 4.455 & -2.019 & \\
\hline HETATM & $103 \mathrm{C}$ & 0 & -3.420 & 4.422 & 1.618 & \\
\hline HETATM & $104 \mathrm{C}$ & 0 & -5.799 & 0.196 & -2.095 & \\
\hline HETATM & $105 \mathrm{C}$ & 0 & -5.527 & 0.039 & 1.494 & \\
\hline HETATM & $106 \mathrm{C}$ & 0 & -5.599 & 1.941 & -0.215 & \\
\hline HETATM & $107 \mathrm{C}$ & 0 & -4.864 & 3.340 & -0.204 & \\
\hline HETATM & $108 \mathrm{~N}$ & 0 & -4.051 & 3.270 & 0.994 & \\
\hline HETATM & $109 \mathrm{~N}$ & 0 & -5.022 & 1.277 & 0.938 & $\gamma$ \\
\hline HETATM & $110 \mathrm{~N}$ & 0 & -5.250 & 1.400 & -1.502 & \\
\hline HETATM & $111 \mathrm{C}$ & 0 & -4.398 & 2.212 & -2.222 & \\
\hline HETATM & $112 \mathrm{~N}$ & 0 & -4.155 & 3.333 & -1.458 & \\
\hline HETATM & $113 \mathrm{C}$ & 0 & -4.104 & 2.046 & 1.607 & \\
\hline HETATM & $114 \mathrm{O}$ & 0 & -4.021 & 2.024 & -3.352 & \\
\hline HETATM & $115 \mathrm{O}$ & 0 & -3.514 & 1.724 & 2.624 & \\
\hline HETATM & $116 \mathrm{H}$ & 0 & -6.719 & -1.777 & -0.310 & \\
\hline HETATM & $117 \mathrm{H}$ & 0 & -5.619 & -3.948 & -0.572 & \\
\hline HETATM & $118 \mathrm{H}$ & 0 & -2.729 & -6.209 & -0.721 & \\
\hline HETATM & $119 \mathrm{H}$ & 0 & -0.355 & -6.777 & -0.727 & \\
\hline HETATM & $120 \mathrm{H}$ & 0 & -4.255 & -5.235 & 1.092 & \\
\hline HETATM & $121 \mathrm{H}$ & 0 & -3.426 & -4.270 & 2.320 & \\
\hline HETATM & $122 \mathrm{H}$ & 0 & -3.184 & -3.701 & -3.338 & \\
\hline HETATM & $123 \mathrm{H}$ & 0 & -4.082 & -4.924 & -2.418 & \\
\hline HETATM & $124 \mathrm{H}$ & 0 & 3.262 & -6.056 & -0.453 & \\
\hline HETATM & $125 \mathrm{H}$ & 0 & 5.192 & -4.603 & -0.088 & \\
\hline HETATM & $126 \mathrm{H}$ & 0 & 6.860 & -1.252 & 0.232 & \\
\hline HETATM & $127 \mathrm{H}$ & 0 & 6.859 & 1.194 & 0.325 & \\
\hline HETATM & $128 \mathrm{H}$ & 0 & 5.856 & -2.959 & 1.786 & \\
\hline HETATM & $129 \mathrm{H}$ & 0 & 4.494 & -2.384 & 2.763 & $\mathrm{H}$ \\
\hline HETATM & $130 \mathrm{H}$ & 0 & 5.134 & -2.238 & -2.884 & \\
\hline HETATM & $131 \mathrm{H}$ & 0 & 6.246 & -2.912 & -1.676 & \\
\hline HETATM & $132 \mathrm{H}$ & 0 & 1.055 & -5.448 & 2.397 & \\
\hline HETATM & $133 \mathrm{H}$ & 0 & 1.393 & -6.656 & 1.146 & \\
\hline HETATM & $134 \mathrm{H}$ & 0 & 1.275 & -4.688 & -3.241 & \\
\hline HETATM & $135 \mathrm{H}$ & 0 & 1.497 & -6.200 & -2.339 & \\
\hline HETATM & $136 \mathrm{H}$ & 0 & 5.270 & 4.558 & 0.198 & \\
\hline HETATM & $137 \mathrm{H}$ & 0 & 3.376 & 6.078 & -0.158 & \\
\hline HETATM & $138 \mathrm{H}$ & 0 & -0.212 & 6.914 & -0.353 & \\
\hline HETATM & $139 \mathrm{H}$ & 0 & -2.610 & 6.430 & -0.234 & \\
\hline HETATM & $140 \mathrm{H}$ & 0 & 1.576 & 6.684 & 1.531 & \\
\hline HETATM & $141 \mathrm{H}$ & 0 & 1.210 & 5.428 & 2.728 & \\
\hline
\end{tabular}




\begin{tabular}{|c|c|c|c|c|}
\hline HETATM $142 \mathrm{H}$ & 0 & 1.282 & 4.796 & -2.924 \\
\hline HETATM $143 \mathrm{H}$ & 0 & 1.572 & 6.292 & -2.014 \\
\hline HETATM $144 \mathrm{H}$ & 0 & 5.839 & 2.788 & 1.993 \\
\hline HETATM $145 \mathrm{H}$ & 0 & 4.434 & 2.166 & 2.877 \\
\hline HETATM $146 \mathrm{H}$ & 0 & 5.184 & 2.372 & -2.753 \\
\hline HETATM $147 \mathrm{H}$ & 0 & 6.304 & 2.957 & -1.507 \\
\hline HETATM $148 \mathrm{H}$ & 0 & -3.225 & 4.193 & -3.045 \\
\hline HETATM $149 \mathrm{H}$ & 0 & -4.050 & 5.340 & -1.971 \\
\hline HETATM $150 \mathrm{H}$ & 0 & -3.220 & 4.164 & 2.644 \\
\hline HETATM $151 \mathrm{H}$ & 0 & -4.120 & 5.246 & 1.571 \\
\hline HETATM $152 \mathrm{H}$ & 0 & -5.564 & 0.241 & -3.146 \\
\hline HETATM $153 \mathrm{H}$ & 0 & -6.870 & 0.187 & -1.945 \\
\hline HETATM $154 \mathrm{H}$ & 0 & -5.190 & -0.014 & 2.514 \\
\hline HETATM $155 \mathrm{H}$ & 0 & -6.609 & 0.072 & 1.462 \\
\hline HETATM $156 \mathrm{H}$ & 0 & -6.670 & 2.014 & -0.105 \\
\hline HETATM $157 \mathrm{H}$ & 0 & -5.539 & 4.181 & -0.145 \\
\hline
\end{tabular}

END

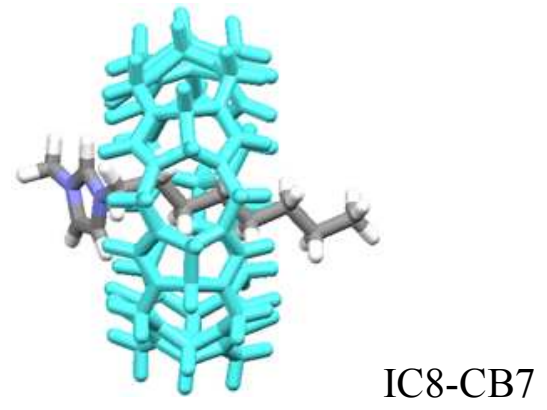

CF Done: $E=-4738.27394111$

REMARK 1 File created by Gauss View 5.0.8

$\begin{array}{llllllll}\text { HETATM } & 1 & \mathrm{C} & 0 & 0.774 & -5.604 & 0.504 & \mathrm{C} \\ \text { HETATM } & 2 & \mathrm{C} & 0 & 2.316 & -5.271 & 0.407 & \mathrm{C} \\ \text { HETATM } & 3 & \mathrm{~N} & 0 & 2.548 & -4.460 & 1.568 & \mathrm{~N} \\ \text { HETATM } & 4 & \mathrm{C} & 0 & 1.387 & -4.115 & 2.228 & \mathrm{C} \\ \text { HETATM } & 5 & \mathrm{~N} & 0 & 0.349 & -4.787 & 1.613 & \mathrm{~N} \\ \text { HETATM } & 6 & \mathrm{~N} & 0 & 0.275 & -5.214 & -0.799 & \mathrm{~N} \\ \text { HETATM } & 7 & \mathrm{C} & 0 & 1.216 & -4.569 & -1.556 & \mathrm{C} \\ \text { HETATM } & 8 & \mathrm{~N} & 0 & 2.401 & -4.589 & -0.871 & \mathrm{~N} \\ \text { HETATM } & 9 & \mathrm{O} & 0 & 1.305 & -3.434 & 3.221 & \mathrm{O} \\ \text { HETATM } & 10 & \mathrm{O} & 0 & 1.062 & -4.138 & -2.684 & \mathrm{O} \\ \text { HETATM } & 11 & \mathrm{C} & 0 & 5.149 & -2.993 & 0.173 & \mathrm{C} \\ \text { HETATM } & 12 & \mathrm{C} & 0 & 5.812 & -1.559 & 0.098 & \mathrm{C} \\ \text { HETATM } & 13 & \mathrm{~N} & 0 & 5.471 & -0.985 & 1.373 & \mathrm{~N} \\ \text { HETATM } & 14 & \mathrm{C} & 0 & 4.717 & -1.818 & 2.169 & \mathrm{C} \\ \text { HETATM } & 15 & \mathrm{~N} & 0 & 4.507 & -2.983 & 1.462 & \mathrm{~N} \\ \text { HETATM } & 16 & \mathrm{~N} & 0 & 4.273 & -2.996 & -0.981 & \mathrm{~N} \\ \text { HETATM } & 17 & \mathrm{C} & 0 & 4.262 & -1.804 & -1.659 & \mathrm{C}\end{array}$




\begin{tabular}{|c|c|c|c|c|c|c|}
\hline HETATM & $18 \mathrm{~N}$ & 0 & 5.178 & -0.972 & -1.069 & $\lambda$ \\
\hline HETATM & $19 \mathrm{O}$ & 0 & 4.390 & -1.611 & 3.312 & $\mathrm{O}$ \\
\hline HETATM & $20 \mathrm{O}$ & 0 & 3.618 & -1.565 & -2.666 & \\
\hline HETATM & $21 \mathrm{C}$ & 0 & 3.853 & -4.111 & 2.095 & $\mathrm{C}$ \\
\hline HETATM & $22 \mathrm{C}$ & 0 & 3.636 & -4.183 & -1.513 & \\
\hline HETATM & $23 \mathrm{C}$ & 0 & 5.672 & 2.099 & 0.069 & $\mathrm{C}$ \\
\hline HETATM & $24 \mathrm{C}$ & 0 & 4.865 & 3.458 & 0.113 & $\mathrm{C}$ \\
\hline HETATM & $25 \mathrm{~N}$ & 0 & 3.977 & 3.252 & 1.236 & $\mathrm{~N}$ \\
\hline HETATM & $26 \mathrm{C}$ & 0 & 4.232 & 2.082 & 1.921 & $\mathrm{C}$ \\
\hline НETATM & $27 \mathrm{~N}$ & 0 & 5.287 & 1.456 & 1.293 & $\mathrm{~N}$ \\
\hline HETATM & $28 \mathrm{~N}$ & 0 & 5.176 & 1.492 & -1.154 & 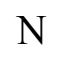 \\
\hline HETATM & $29 \mathrm{C}$ & 0 & 4.394 & 2.341 & -1.898 & $\mathrm{C}$ \\
\hline HETATM & $30 \mathrm{~N}$ & 0 & 4.243 & 3.506 & -1.190 & $\mathrm{~N}$ \\
\hline HETATM & 310 & 0 & 3.693 & 1.721 & 2.939 & $\mathrm{O}$ \\
\hline HETATM & $32 \mathrm{O}$ & 0 & 3.977 & 2.123 & -3.018 & $\mathrm{O}$ \\
\hline HETATM & $33 \mathrm{C}$ & 0 & 1.891 & 5.517 & 0.036 & $\mathrm{C}$ \\
\hline HETATM & $34 \mathrm{C}$ & 0 & 0.339 & 5.802 & -0.082 & $\mathrm{C}$ \\
\hline HETATM & $35 \mathrm{~N}$ & 0 & -0.193 & 5.205 & 1.114 & $\mathrm{~N}$ \\
\hline HETATM & $36 \mathrm{C}$ & 0 & 0.744 & 4.486 & 1.823 & $\mathrm{C}$ \\
\hline HETATM & $37 \mathrm{~N}$ & 0 & 1.962 & 4.666 & 1.200 & $\mathrm{~N}$ \\
\hline HETATM & $38 \mathrm{~N}$ & 0 & 2.202 & 4.885 & -1.226 & 1 \\
\hline HETATM & $39 \mathrm{C}$ & 0 & 1.084 & 4.603 & -1.971 & $\mathrm{C}$ \\
\hline HETATM & $40 \mathrm{~N}$ & 0 & -0.384 & -1.909 & -4.288 & 1 \\
\hline HETATM & $41 \mathrm{C}$ & 0 & -1.484 & -1.077 & -4.101 & $\mathrm{C}$ \\
\hline HETATM & $42 \mathrm{C}$ & 0 & -1.048 & 0.011 & -3.447 & $\mathrm{C}$ \\
\hline HETATM & $43 \mathrm{~N}$ & 0 & 0.313 & -0.179 & -3.220 & $\mathrm{~N}$ \\
\hline HETATM & $44 \mathrm{C}$ & 0 & 0.676 & -1.348 & -3.728 & $\mathrm{C}$ \\
\hline HETATM & $45 \mathrm{C}$ & 0 & 1.228 & 0.771 & -2.536 & $\mathrm{C}$ \\
\hline HETATM & $46 \mathrm{C}$ & 0 & 1.395 & 0.428 & -1.042 & $\mathrm{C}$ \\
\hline HETATM & $47 \mathrm{C}$ & 0 & 0.160 & 0.670 & -0.148 & $\mathrm{C}$ \\
\hline HETATM & $48 \mathrm{C}$ & 0 & 0.465 & 0.278 & 1.321 & $\mathrm{C}$ \\
\hline HETATM & $49 \mathrm{C}$ & 0 & -0.429 & -3.196 & -5.011 & $\mathrm{C}$ \\
\hline HETATM & $50 \mathrm{C}$ & 0 & -0.717 & 0.525 & 2.284 & $\mathrm{C}$ \\
\hline HETATM & $51 \mathrm{C}$ & 0 & -0.379 & 0.064 & 3.721 & $\mathrm{C}$ \\
\hline HETATM & $52 \mathrm{C}$ & 0 & -1.555 & 0.322 & 4.691 & $\mathrm{C}$ \\
\hline HETATM & $53 \mathrm{C}$ & 0 & -1.195 & -0.087 & 6.139 & $\mathrm{C}$ \\
\hline HETATM & $54 \mathrm{H}$ & 0 & -2.025 & 0.092 & 6.813 & $\mathrm{H}$ \\
\hline HETATM & $55 \mathrm{H}$ & 0 & -0.342 & 0.476 & 6.497 & $\mathrm{H}$ \\
\hline HETATM & $56 \mathrm{H}$ & 0 & -0.945 & -1.140 & 6.183 & $\mathrm{H}$ \\
\hline HETATM & $57 \mathrm{H}$ & 0 & -2.413 & -0.238 & 4.355 & $\mathrm{H}$ \\
\hline HETATM & $58 \mathrm{H}$ & 0 & -1.821 & 1.370 & 4.658 & $\mathrm{H}$ \\
\hline HETATM & $59 \mathrm{H}$ & 0 & 0.499 & 0.600 & 4.061 & $\mathrm{H}$ \\
\hline HETATM & $60 \mathrm{H}$ & 0 & -0.142 & -0.992 & 3.713 & $\mathrm{H}$ \\
\hline HETATM & $61 \mathrm{H}$ & 0 & -1.592 & -0.015 & 1.941 & $\mathrm{H}$ \\
\hline HETATM & $62 \mathrm{H}$ & 0 & -0.955 & 1.579 & 2.306 & $\mathrm{H}$ \\
\hline HETATM & $63 \mathrm{H}$ & 0 & 1.322 & 0.836 & 1.679 & $\mathrm{H}$ \\
\hline
\end{tabular}




\begin{tabular}{|c|c|c|c|c|c|c|}
\hline HETATM & $64 \mathrm{H}$ & 0 & 0.734 & -0.772 & 1.356 & $\mathrm{H}$ \\
\hline HETATM & $65 \mathrm{H}$ & 0 & -0.678 & 0.087 & -0.510 & $\mathrm{H}$ \\
\hline HETATM & $66 \mathrm{H}$ & 0 & -0.123 & 1.715 & -0.197 & \\
\hline HETATM & $67 \mathrm{H}$ & 0 & 2.213 & 1.028 & -0.664 & \\
\hline HETATM & $68 \mathrm{H}$ & 0 & 1.697 & -0.607 & -0.965 & \\
\hline HETATM & $69 \mathrm{H}$ & 0 & 0.834 & 1.760 & -2.692 & \\
\hline HETATM & $70 \mathrm{H}$ & 0 & 2.179 & 0.714 & -3.030 & \\
\hline HETATM & $71 \mathrm{H}$ & 0 & 0.493 & -3.717 & -4.843 & \\
\hline HETATM & $72 \mathrm{H}$ & 0 & -0.578 & -3.001 & -6.063 & \\
\hline НETATM & $73 \mathrm{H}$ & 0 & -1.245 & -3.764 & -4.604 & \\
\hline HETATM & $74 \mathrm{H}$ & 0 & 1.654 & -1.759 & -3.653 & \\
\hline HETATM & $75 \mathrm{H}$ & 0 & -1.556 & 0.899 & -3.160 & \\
\hline HETATM & $76 \mathrm{H}$ & 0 & -2.464 & -1.352 & -4.392 & \\
\hline HETATM & $77 \mathrm{~N}$ & 0 & -0.001 & 5.136 & -1.323 & \\
\hline HETATM & $78 \mathrm{O}$ & 0 & 0.554 & 3.892 & 2.857 & \\
\hline HETATM & $79 \mathrm{O}$ & 0 & 1.066 & 4.053 & -3.054 & \\
\hline HETATM & $80 \mathrm{C}$ & 0 & 3.188 & 4.293 & 1.871 & $\mathrm{C}$ \\
\hline HETATM & $81 \mathrm{C}$ & 0 & 3.535 & 4.643 & -1.746 & \\
\hline HETATM & $82 \mathrm{C}$ & 0 & 5.928 & 0.295 & 1.874 & $\mathrm{C}$ \\
\hline HETATM & $83 \mathrm{C}$ & 0 & 5.641 & 0.243 & -1.722 & \\
\hline HETATM & $84 \mathrm{C}$ & 0 & -3.163 & 4.922 & -0.267 & \\
\hline HETATM & $85 \mathrm{C}$ & 0 & -4.403 & 3.939 & -0.306 & \\
\hline HETATM & $86 \mathrm{~N}$ & 0 & -4.375 & 3.335 & 1.001 & $\mathrm{~N}$ \\
\hline HETATM & $87 \mathrm{C}$ & 0 & -3.298 & 3.731 & 1.764 & \\
\hline HETATM & $88 \mathrm{~N}$ & 0 & -2.594 & 4.663 & 1.031 & $\mathrm{~N}$ \\
\hline HETATM & $89 \mathrm{~N}$ & 0 & -2.375 & 4.487 & -1.400 & \\
\hline HETATM & $90 \mathrm{C}$ & 0 & -2.880 & 3.370 & -2.008 & \\
\hline HETATM & $91 \mathrm{~N}$ & 0 & -4.078 & 3.066 & -1.416 & \\
\hline HETATM & $92 \mathrm{O}$ & 0 & -3.068 & 3.400 & 2.902 & \\
\hline HETATM & $93 \mathrm{O}$ & 0 & -2.398 & 2.806 & -2.973 & \\
\hline HETATM & $94 \mathrm{C}$ & 0 & -5.943 & 0.630 & -0.167 & \\
\hline HETATM & $95 \mathrm{C}$ & 0 & -5.891 & -0.944 & -0.007 & \\
\hline HETATM & $96 \mathrm{~N}$ & 0 & -5.243 & -1.107 & 1.270 & \\
\hline HETATM & $97 \mathrm{C}$ & 0 & -4.981 & 0.085 & 1.909 & $\mathrm{C}$ \\
\hline HETATM & $98 \mathrm{~N}$ & 0 & -5.398 & 1.102 & 1.081 & $\mathrm{~N}$ \\
\hline HETATM & $99 \mathrm{~N}$ & 0 & -5.139 & 0.849 & -1.352 & \\
\hline HETATM & $100 \mathrm{C}$ & 0 & -4.703 & -0.311 & -1.938 & \\
\hline HETATM & $101 \mathrm{~N}$ & 0 & -5.143 & -1.363 & -1.174 & \\
\hline HETATM & $102 \mathrm{O}$ & 0 & -4.562 & 0.209 & 3.035 & \\
\hline HETATM & $103 \mathrm{O}$ & 0 & -4.121 & -0.394 & -3.002 & \\
\hline HETATM & $104 \mathrm{C}$ & 0 & -5.406 & 2.476 & 1.548 & \\
\hline HETATM & $105 \mathrm{C}$ & 0 & -4.995 & 2.123 & -2.026 & \\
\hline HETATM & $106 \mathrm{C}$ & 0 & -1.524 & 5.427 & 1.644 & \\
\hline HETATM & $107 \mathrm{C}$ & 0 & -1.282 & 5.234 & -1.992 & \\
\hline HETATM & $108 \mathrm{C}$ & 0 & -5.000 & -2.734 & -1.623 & \\
\hline HETATM & $109 \mathrm{C}$ & 0 & -5.057 & -2.364 & 1.962 & \\
\hline
\end{tabular}




\begin{tabular}{|c|c|c|c|c|}
\hline HETATM 110 & 0 & -1.030 & -5.567 & -1.328 \\
\hline HETATM 111 & 0 & -0.937 & -4.899 & 2.272 \\
\hline HETATM 112 & 0 & -2.755 & -4.953 & 0.467 \\
\hline HETATM 113 & 0 & -4.080 & -4.099 & 0.351 \\
\hline HETATM 114 I & 0 & -3.981 & -3.196 & 1.464 \\
\hline HETATM $115 \mathrm{~N}$ & 0 & -2.068 & -4.326 & 1.569 \\
\hline HETATM 116 N & 0 & -2.154 & -4.794 & -0.840 \\
\hline HETATM 117 C & 0 & -2.860 & -3.948 & -1.660 \\
\hline HETATM 118 N & 0 & -3.962 & -3.513 & -0.971 \\
\hline HETATM 119 C & 0 & -2.787 & -3.297 & 2.145 \\
\hline HETATM 120 & 0 & -2.612 & -3.711 & -2.827 \\
\hline HETATM $121 \mathrm{C}$ & 0 & -2.474 & -2.676 & 3.131 \\
\hline HETATM $122 \mathrm{H}$ & 0 & 0.566 & -6.648 & 0.687 \\
\hline HETATM $123 \mathrm{H}$ & 0 & 2.950 & -6.145 & 0.411 \\
\hline HETATM $124 \mathrm{H}$ & 0 & 5.861 & -3.801 & 0.101 \\
\hline HETATM $125 \mathrm{H}$ & 0 & 6.882 & -1.585 & -0.039 \\
\hline HETATM $126 \mathrm{H}$ & 0 & 4.492 & -4.981 & 2.021 \\
\hline HETATM $127 \mathrm{H}$ & 0 & 3.715 & -3.832 & 3.127 \\
\hline HETATM $128 \mathrm{H}$ & 0 & 3.396 & -3.987 & -2.543 \\
\hline HETATM $129 \mathrm{H}$ & 0 & 4.344 & -5.000 & -1.447 \\
\hline HETATM $130 \mathrm{H}$ & 0 & 6.742 & 2.231 & 0.021 \\
\hline HETATM $131 \mathrm{H}$ & 0 & 5.489 & 4.326 & 0.267 \\
\hline HETATM $132 \mathrm{H}$ & 0 & 2.486 & 6.408 & 0.170 \\
\hline HETATM $133 \mathrm{H}$ & 0 & 0.093 & 6.852 & -0.132 \\
\hline HETATM $134 \mathrm{H}$ & 0 & 3.808 & 5.177 & 1.978 \\
\hline HETATM $135 \mathrm{H}$ & 0 & 2.906 & 3.915 & 2.840 \\
\hline HETATM $136 \mathrm{H}$ & 0 & 3.426 & 4.451 & -2.800 \\
\hline HETATM $137 \mathrm{H}$ & 0 & 4.131 & 5.531 & -1.582 \\
\hline HETATM $138 \mathrm{H}$ & 0 & 5.701 & 0.298 & 2.928 \\
\hline HETATM $139 \mathrm{H}$ & 0 & 6.994 & 0.381 & 1.716 \\
\hline HETATM $140 \mathrm{H}$ & 0 & 5.281 & 0.221 & -2.736 \\
\hline HETATM $141 \mathrm{H}$ & 0 & 6.724 & 0.231 & -1.711 \\
\hline HETATM $142 \mathrm{H}$ & 0 & -3.432 & 5.963 & -0.373 \\
\hline НЕTATM $143 \mathrm{H}$ & 0 & -5.345 & 4.435 & -0.482 \\
\hline НЕTATM $144 \mathrm{H}$ & 0 & -6.941 & 1.014 & -0.316 \\
\hline HETATM $145 \mathrm{H}$ & 0 & -6.866 & -1.408 & -0.005 \\
\hline HETATM $146 \mathrm{H}$ & 0 & -6.372 & 2.911 & 1.329 \\
\hline HETATM $147 \mathrm{H}$ & 0 & -5.240 & 2.437 & 2.612 \\
\hline HETATM $148 \mathrm{H}$ & 0 & -4.616 & 1.907 & -3.012 \\
\hline HETATM $149 \mathrm{H}$ & 0 & -5.968 & 2.592 & -2.094 \\
\hline HETATM $150 \mathrm{H}$ & 0 & -1.752 & 6.482 & 1.556 \\
\hline HETATM $151 \mathrm{H}$ & 0 & -1.502 & 5.132 & 2.681 \\
\hline HETATM $152 \mathrm{H}$ & 0 & -1.135 & 4.844 & -2.986 \\
\hline HETATM $153 \mathrm{H}$ & 0 & -1.574 & 6.276 & -2.037 \\
\hline HETATM $154 \mathrm{H}$ & 0 & -4.745 & -2.701 & -2.669 \\
\hline HETATM $155 \mathrm{H}$ & 0 & -5.948 & -3.237 & -1.486 \\
\hline
\end{tabular}




$\begin{array}{llllrrr}\text { HETATM } 156 \mathrm{H} & 0 & -4.819 & -2.114 & 2.982 & \mathrm{H} \\ \text { HETATM } & 157 \mathrm{H} & 0 & -5.977 & -2.934 & 1.916 & \mathrm{H} \\ \text { HETATM } & 158 \mathrm{H} & 0 & -0.989 & -5.419 & -2.393 & \mathrm{H} \\ \text { HETATM } 159 \mathrm{H} & 0 & -1.208 & -6.610 & -1.104 & \mathrm{H} \\ \text { HETATM 160 H } & 0 & -0.852 & -4.368 & 3.206 & \mathrm{H} \\ \text { HETATM 161 H } & 0 & -1.141 & -5.948 & 2.455 & \mathrm{H} \\ \text { HETATM 162 H } & 0 & -2.934 & -5.998 & 0.671 & \mathrm{H} \\ \text { HETATM 163 H } & 0 & -4.981 & -4.691 & 0.417 & \mathrm{H} \\ \text { END } & & & & & & \end{array}$

\section{References}

[1] S. Liu, C. Ruspic, P. Mukhopadhyay, S. Chakrabarti, P. Y. Zavalij, L. Isaacs, J. Am. Chem. Soc. 2005, 127, 15959-15967.

[2] T. L. Amyes, S. T. Diver, J. P. Richard, F. M. Rivas, K. Toth, J. Am. Chem. Soc. 2004, 126, 4366-4374.

[3] S. T. Handy, M. Okello, J. Org. Chem. 2005, 70, 1915-1918.

[4] L. P. B. Paul Haake, Walter B. Miller, J. Am. Chem. Soc. 1969, 91. 